University of Louisville

ThinkIR: The University of Louisville's Institutional Repository

Electronic Theses and Dissertations

$12-2018$

\title{
Student engagement in post-soviet higher education: coping with academic quality problems.
}

\author{
J. L. Mettille \\ University of Louisville
}

Follow this and additional works at: https://ir.library.louisville.edu/etd

Part of the Scholarship of Teaching and Learning Commons

\section{Recommended Citation}

Mettille, J. L., "Student engagement in post-soviet higher education: coping with academic quality problems." (2018). Electronic Theses and Dissertations. Paper 3116.

https://doi.org/10.18297/etd/3116

This Doctoral Dissertation is brought to you for free and open access by ThinkIR: The University of Louisville's Institutional Repository. It has been accepted for inclusion in Electronic Theses and Dissertations by an authorized administrator of ThinkIR: The University of Louisville's Institutional Repository. This title appears here courtesy of the author, who has retained all other copyrights. For more information, please contact thinkir@louisville.edu. 


\title{
STUDENT ENGAGEMENT IN POST-SOVIET HIGHER EDUCATION: COPING WITH ACADEMIC QUALITY PROBLEMS
}

\author{
By \\ J. L. Mettille \\ B.A., Biology and Psychology, 2007 \\ Saint Louis University \\ M.A., Higher Education Administration, 2011 \\ University of Louisville
}

\begin{abstract}
A Dissertation
Submitted to the Faculty of the

College of Education and Human Development of the University of Louisville in Partial Fulfillment of the Requirements

for the Degree of
\end{abstract}

Doctor of Philosophy in Educational Leadership and Organizational Development

Department of Educational Leadership, Evaluation, and Organizational Development University of Louisville

Louisville, Kentucky

December 2018 
Copyright (C) 2018 by J. L. Mettille

All rights reserved 

STUDENT ENGAGEMENT IN POST-SOVIET HIGHER EDUCATION:

COPING WITH ACADEMIC QUALITY PROBLEMS

\author{
By \\ J. L. Mettille \\ B.A., Biology and Psychology, 2007 \\ Saint Louis University \\ M.A., Higher Education Administration, 2011 \\ University of Louisville
}

A Dissertation Approved on

12 November 2018

by the following Dissertation Committee:

Dissertation Director
Dr. Jacob P. Gross

Dr. Amy S. Hirschy

Dr. Meghan J. Pifer

Dr. Jeffrey C. Sun 


\section{DEDICATION}

This dissertation is dedicated to the university administrators, faculty, and students in the Kyrgyz Republic who made this dissertation possible by generously letting me into their lives and sharing their stories. I am especially grateful to Mukadas Tashieva

Abdibaitovna, Kulkharai Abdurakhmanova Tulanbaevna, and Sabyrkul Kalygulova Shamsitdinovna for their relentless optimism and unconditional support over the years it has taken to complete this study. I look forward to returning to Kyrgyzstan and working with these talented individuals as we continue to improve lives throughout Central Asia by empowering learners to pursue their dreams and realize their professional ambitions. 


\section{ACKNOWLEDGMENTS}

The number of people to whom I am indebted for their support and encouragement over the seven years it has taken to complete this dissertation far exceed this single page allotment. I cannot begin to thank my dissertation chair and committee enough for their dedication to this project. Dr. Jacob Gross showed impressive patience in dealing with the inevitable impasses and difficulties. Dr. Amy Hirschy reminded me to keep rowing, even when the shore appeared to keep moving further away. Dr. Meghan Pifer and Dr. Jeffrey Sun joined and contributed to a committee that has had a rotating cast and a changing story over the years. I must thank my parents Dr. Shayla Damron and John Mettille for supporting me throughout this endeavor and tolerating the setbacks in this process. I thank Dr. Juan Arturo Herrera Ortiz for helping me find my way out of a very hopeless place. I commend my best friend Dr. Caroline Amanda McDonald for listening without judging and enthusiastically encouraging me go to the moon when necessary, and sometimes even when not. Finally, this degree and study would not have been possible without the advice, guidance, and genuine friendship of Dr. Susan Lasky. I consider myself lucky to have met Dr. Lasky in my first graduate course at the University of Louisville and take pride that I followed through on her encouragement to continue pursuing this doctorate no matter the setbacks. This dissertation followed me from Seattle to Riyadh and back again, and I am thrilled that this journey is finally concluding and look forward to whatever the future may bring. 


\title{
ABSTRACT \\ STUDENT ENGAGEMENT IN POST-SOVIET HIGHER EDUCATION: COPING WITH ACADEMIC QUALITY PROBLEMS
}

\author{
J. L. Mettille
}

12 November 2018

This dissertation investigates the experiences of administrators, instructional faculty, and students within a post-Soviet higher education institution in the Kyrgyz Republic using qualitative field research and case study methods. This study identifies the perceived potential of Western benchmarks of effective educational practice to evince positive change in the institution as well as their propriety and relevance in this context. In addition, the propositions underlying the student engagement construct and overarching the benchmarks are evaluated based upon participant responses and classroom observation data. Twenty higher education practitioners and twenty students participated in semi-structured interviews preceding and following seven classroom observations. The recorded interview data was transcribed then analyzed using first cycle and second cycle coding procedures to elicit predominant ethnographic themes within the case, specifically the teaching and learning environment within the university program under analysis. Data condensation via coding, display through narrative, matrix analysis and participant vignettes, and interpretation prior to conclusion drawing and verification leant analytical rigor to findings. The resulting themes centered around three central 
tenets within the ethnographic case: fear, freedom, and independence. Their interpretation and verification leant additional strength to conclusions indicating a lack of evidence supporting implementation of the benchmarks, logistical challenges to such alterations, and deeper philosophical disconnects which could potentially impact the success or failure of such initiatives. The data was then used to justify implications for policy, practice, and research all emphasizing critical analysis and reflection upon the evidence base that the student engagement construct is dependent upon. 
TABLE OF CONTENTS

PAGE

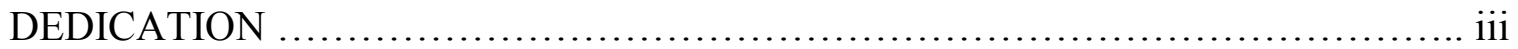

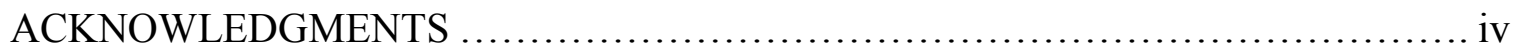

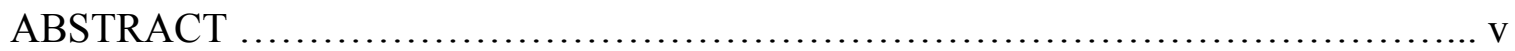

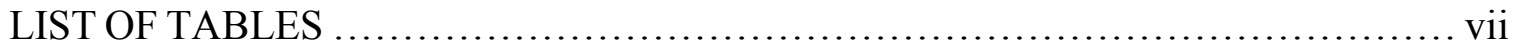

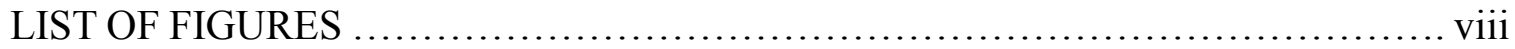

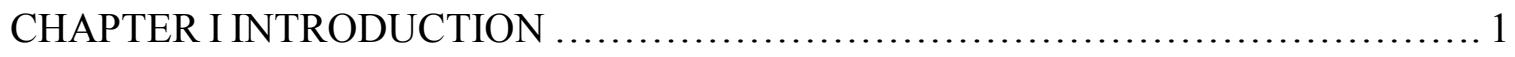

CHAPTER II LITERATURE REVIEW ............................................. 31

CHAPTER III METHODS ….................................................. 79

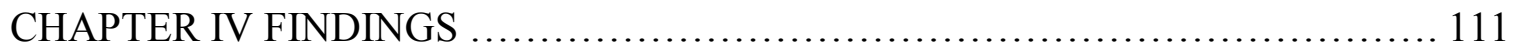

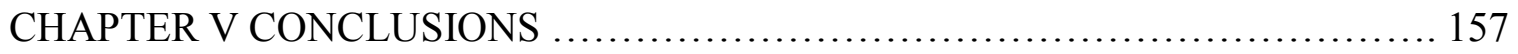

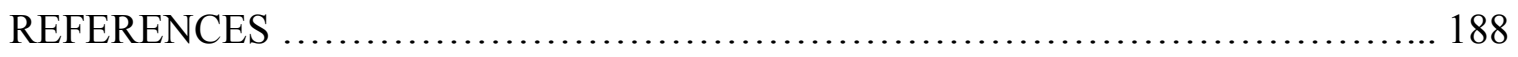

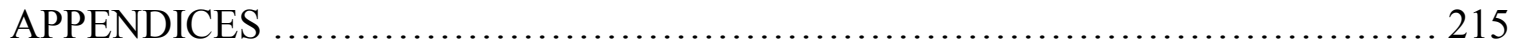

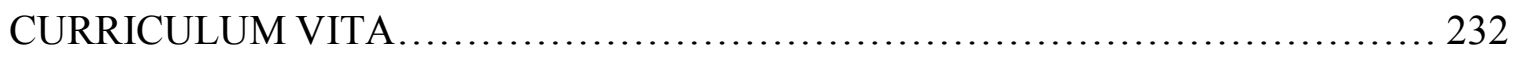




\section{LIST OF TABLES}

TABLE PAGE

1. A Comparison of Qualitative and Quantitative Means for Promoting Analytical Rigor with Study Application........................................................... 104

2. Reflections and Perceptions of Educational Conditions and Practices Supporting Student Engagement among Practitioner and Student Participants................... 141

3. Crosswalk of Ethnographic Themes, Codes, and Research Sub-Questions........... 149 


\section{LIST OF FIGURES}

FIGURE

PAGE

1. Theoretical and Practical Contexts............................................ 19

2. Higher Education Evidence-Based Practice................................... 20

3. Learning and Teaching Environment Ethnographic Case......................... 22

4. Research Site Map..................................................... 23

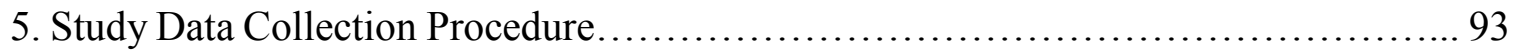

6. Ethnographic Overview and Qualitative Content Analysis Procedures................ 96

7. First Cycle and Second Cycle Coding Procedures and Outcomes..................... 97

8. First Cycle First Interview Codes....................................... 120

9. First Cycle In-Vivo Classroom Observation Codes............................ 128

10. First Cycle In-Vivo and Values-Based Second Interview Codes................... 131

11. Secondary Code Analysis for Ethnographic Themes................................. 136 


\section{CHAPTER I}

\section{INTRODUCTION}

This dissertation investigates the perceptions of Western evidence-based practices among post-Soviet higher education practitioners through a qualitative ethnographic case study. While completing a teaching fellowship in 2017, I observed the conditions, challenges, and realities of life and work for individuals in the Kyrgyz Republic (KR). As a post-Soviet nation in Central Asia, the KR continues to face difficulties stemming from its independence roughly thirty years ago. With few natural resources and limited international support, this small nation is struggling to define itself and participate in the global economy while affording basic services and rights to its citizens (International Crisis Group, 2016). As a teaching fellow, I observed that the experiences of administrators, faculty, and students in the KR reflect its multiethnic culture and the encompassing post-Soviet context. Modern public education in the KR remains a vestige of Soviet governance and command economy planning. Its curricula, facilities, beliefs, and practices have needed to adapt to the changing socioeconomic conditions and operational challenges inherent to a developing market economy but have failed to do so.

The teaching fellowship I completed seeks to improve the quality of English as a foreign language (EFL) education by enhancing pre-service teacher programs and inservice teacher instruction through professional development and intensive English language training at select sites throughout the world. A public university hosts the 
fellowship annually in Osh, the largest city in southern KR, and fellows are known regionally for training interpreters, students, and foreign language teachers. External organizations consistently provide fellows, specialists, and volunteers throughout Osh to assist teachers in promoting foreign language accuracy and proficiency. However, university graduates throughout the region visibly fail to develop language abilities for basic communication, much less adequate linguistic and methodological skills for instruction. Consultants and external parties have struggled to evince change in university programs, and stakeholder progress appears elusive. While the KR continues to transition economically, socially, and politically, the facilities and quality of the national public education system continue to deteriorate (Mertaugh, 2004). Poor educational quality, substandard teaching, and inadequate learning form a cycle that has proven to be particularly difficult to interrupt in this context (Merrill, Yakubova, \& Turlanbekova, 2015). Improving student outcomes and advancing national interests is a global challenge that even developed nations with modern economies struggle to facilitate (Freedberg, 2017). This dissertation explores the professional experiences of post-Soviet higher education practitioners (faculty and administrators) and students before detailing their perceptions of Western benchmarked practices designed to promote student engagement. This study garners ground-level evidence essential for the identification of potentially effective means for improving higher education quality within the developing and rapidly changing context of the KR.

\section{Fellowship Experience}

The impetus for this dissertation emerged from the teaching fellowship I completed at the research site in the south of the KR in July of 2017. My fellowship 
responsibilities included conducting educational needs assessments, providing expertise on EFL instruction, and consulting on institutional and systemic higher education policies and practices. I pursued these responsibilities while supporting the intents and objectives of a sponsoring external government agency. An initial needs assessment revealed extensive problems at the institution for teacher preparation: students lacked basic academic skills, faculty were untrained and unsupported, and most facilities lacked instructional resources and technologies. While students and junior faculty readily acknowledged certain deficits within the program, senior faculty and administrators generally evaded discussion and dismissed curricular and instructional concerns.

I realized that the existing official data were clearly inaccurate. Basic information, such as student enrollment and class attendance, could not reflect reality given that institutional facilities could not possibly accommodate even one-tenth of the purported student population. Administrative policies and procedures therefore lacked accurate operational data, and this institution's administration and student outcomes suffered accordingly. I observed English language proficiency and accuracy to be critically low, if not wholly absent, among some program graduates and faculty. Months of immersion within the research site revealed that persistently low educational quality, and correspondingly negative student outcomes were endemic to most levels of the national public education system. I noticed an entrenched cycle of pre-service teachers learning from ineffective faculty to ineffectively teach content and skills that they themselves lacked. I opted to re-focus my teaching fellowship on interrupting this cycle.

The host university additionally expected me, as the teaching fellow, to further the professional development of instructional faculty members and program administrators. I 
produced a series of workshops that presented the findings of the needs assessment, explained how to implement data-based processes for assessing student learning, and provided training on effective teaching practices from What the Best College Teachers Do (Bain, 2004). Participants were receptive to the general findings of the needs assessment and supported the idea of data-driven student performance assessments and multiple levels of language instruction. However, senior faculty members emphatically resisted the application of evidence-based practices to instructional praxis. Clearly, something went wrong in the way I proposed empirically validated teaching methods and administration. These strongly negative feelings presented a bit of a mystery; students were receptive to new practices in class, and faculty had conceded that the learning conditions were substandard, student outcomes were poor, and systems and structures needed to change.

From these concessions, I expected it to be self-evident that actual teaching practices needed to change before student outcomes would improve. The proposed changes drew their empirical support from the teaching attitudes, experiences, and student-centered practices of outstanding university-level faculty from throughout the United States (Bain, 2004). At that time, I did not know the extent of the philosophical and practical disconnects between Western-derived educational practices and the traditional techniques used throughout this post-Soviet higher education institution. My workshops presenting Western-based post-secondary instructional techniques simply did not translate into the mindsets or realities of these well-intentioned faculty members. By focusing on promoting changes to instruction, I had also unintentionally associated the state of the program and its outcomes with faculty behaviors and praxis. In other words, I 
had essentially blamed the program's state on the faculty, including those who were willing to participate, and those with whom I was partnering to make improvements. Unfortunately, I was (at the time of my fellowship) unable to understand the roles that administrators, students, or the institution itself played in perpetuating substandard education. In addition, I did not account or plan for the institution's context and the daunting external and internal forces unique to post-Soviet life.

In reflecting on my fellowship and the difficulties I faced in evincing change, it became apparent that the relationship between Western-based educational practice and post-Soviet praxis warranted further analysis to ensure the efficacy of future efforts. I suspected that by analyzing administrator and faculty perceptions of Western evidencebased practice within the realities of the post-Soviet KR context, I could reduce the disconnects I had observed and mitigate the pushback I had experienced. This study strives to contribute to future educational quality improvement reform in developing economies, and specifically in post-Soviet contexts, by identifying responsive and sensitive means for supporting desired student outcomes. Thus, this dissertation explores the perceived practical potential of Western benchmarks of effective educational practice within post-Soviet higher education praxis.

\section{Dissertation Overview}

Chapter One provides an account of my fellowship experience that led to the dissertation and introduces the background and context of the research site. After, it details the education and research problems, and highlights the potential of student engagement via reflective analysis. I provide an outline of the research questions, theoretical framework, operational definitions, study design, research methods, 
assumptions, and delimitations for the analysis. The discussion relates the study to audiences and proposes contributions to theory and practice. Chapter Two examines aspects of life and education in pre-Soviet Central Asia, Soviet Kyrgyzstan, and the postSoviet KR, specifically analyzing the context of the research site and aspects of educational practice. It covers previous attempts at education reform within the KR as well as current national conditions. Next, I provide an overview of the body of literature on student success which provided a foundation for the development of the student engagement construct. I then detail student engagement from its conceptual and theoretical underpinnings to its practical applications, modern critiques, and international interpretations. The chapter concludes by introducing the theoretical foundation guiding the study - specifically, the benchmarked educational practices associated with the National Survey of Student Engagement (NSSE). Chapter Three provides further analysis of the individual NSSE benchmarks and their applications within international contexts before explaining the rationale behind the ethnographic case study design and qualitative research methods. Chapter Four presents the body of empirical information collected from the investigation of the ethnographic case and study participants. Finally, Chapter Five summarizes the major findings of the study, further discusses analytical constraints, presents implications of the findings relative to theory, practice, and research, and concludes the dissertation.

\section{Background}

This study explores evidence-based practices associated with student engagement within a post-secondary teacher preparation program in the KR. Specifically, I investigate higher education practitioner and student experiences and perceptions of 
Western-derived instructional practices within post-Soviet higher education praxis throughout the analysis. This introduction provides context for the study via brief discussion of the historical development of and socioeconomic conditions at the research site in the KR. First, the introduction highlights the nation's current fragile state and the declining quality of its education system. Second, the introduction focuses on previous attempts at education reform in the KR and substantiates the national need for improved education at all levels. Third, the introduction centers on the potential of the student engagement construct, exploring its assessment and use in international contexts and further rationalizing the need for reflective analysis of its practical potential at the research site. Finally, the introduction approaches the ethnographic case study design and qualitative research methods, relates the discussion to economic development and education reform audiences, and identifies potential contributions to higher education research, theory, and practice.

Preliminary evidence from my fellowship experiences revealed that many assumptions inherent to Western higher education are irrelevant to the realities of life within developing nations. While Western education is predicated on the belief that successful education leads to personal development, fulfillment, and opportunity, these traits are not always priorities in the developing countries, governments, and economies found throughout post-Soviet Central Asia (Heyneman \& DeYoung, 2004). Even within the United States, reality sometimes falls short of the American ideals of individualism and opportunity driven by talent and dedication within a meritocratic society (Thelin, 2007). Thelin (2007) conceded that the distance between democratic dreams and realities highlight the perceived yet flawed importance of American higher education for 
advancing social mobility. Current research supports this observation, providing substantial evidence indicating that college completion remains highly correlated with student-level variables including race and ethnicity, gender, and age despite targeted institutional and governmental directives (Shapiro, 2017). I inferred that perhaps the expectations for higher education in the KR were simply more aligned with reality and corresponding outcomes compared to those harbored by students in the United States.

Former Soviet rule created national systems of compulsory public education to support planned economic development and social advancement efforts while restricting higher education to the elites (DeYoung, 2004). Following its independence, the KR has achieved near-universal access to higher education at the expense of educational standards and quality assurance at all levels (Smolentseva, 2012). With the collapse of the Soviet Union, many economic and social supports disappeared, and poverty increased drastically (Anderson \& Pomfret, 2000). For many people within the KR, survival has taken precedence over education and personal development (Kuehnast, 2002). My teaching fellowship reflected this understanding of poverty: I visited rural schools and communities where a survivalist mentality predominated, and subsistence farming, seminomadic herding, and trading were prioritized over youth attaining a basic education, much less pursuing further education. I interpreted this mentality as contradicting Western beliefs in the legitimacy and strength of the individual relative to ability, talent, and opportunity. Such beliefs also coincide with Thelin's (2007) postulation that "life choices more often than not are mere probabilities shaped by collective forces outside our control" (p. 59). I argue that individuals in the KR who overcome tremendously challenging economic and sociopolitical circumstances to pursue higher education 
deserve a degree of academic quality and effectiveness, standards that should define basic university practice and student rights.

\section{Purpose Statement}

The purpose of this dissertation is the exploration of practitioner and student experiences and perceptions of higher education practices linked with student engagement. This purpose includes the evaluation of Western higher education practice potential in a post-secondary institution operating within a post-Soviet context. The KR has an extensive history of education reform and counter-reform, which I will discuss more thoroughly in the literature review to contextualize this dissertation. Modern education reforms in the KR have traditionally been anchored to Western perspectives, assumptions, and beliefs about the role of education for individuals and societies including: democratic pedagogy, learner-centeredness, and individual autonomy (Elliott \& Tudge, 2007a; Tabulawa, 2003). Educational reform must conceptualize and operationalize the context of a developing country, especially one characterized by economic scarcity and a survivalist mentality, before implementing any reforms (Robinson \& Winthrop, 2016). This study explores the perceptions of educational practices associated with the student engagement construct within a specific example of post-Soviet higher education. By fostering reflective analysis among practitioners and students, a greater understanding of the Kyrgyz higher education experience develops, and practitioner perceptions of Western-based education practices evaluate the potential of these practices to improve educational quality at the research site. The following section details threats to national stability in the KR and highlights the extent of educational quality problems to set the stage for the dissertation research problem. 
The collapse of the Soviet Union immediately transformed dependent states into newly independent nations, struggling to define their identities and statehood, develop economically, and survive politically in the rapidly changing post-Soviet context (Everett-Heath, 2003; Heyneman \& DeYoung, 2004; Omelicheva, 2015; Silova, 2011; Starr, 2011). After thirty years of social transitions within Central Asia, former Soviet states have loosely aligned various policies within the Commonwealth of Independent States (CIS) (Khazanov, 1995). States have diversified politically and socially as they established various systems of governance, instituted economic reforms, and advocated for a return to native languages, cultural traditions, and religious customs (Everett-Heath, 2003). However, the efficacy and sustainability of these efforts varies throughout the CIS region, and considerable challenges persist (Drummond \& DeYoung, 2003; Silova, 2011). Some international organizations provide developmental support and financial assistance to CIS constituents with the goal of minimizing threats to member states' national security, sovereignty, and stability (Hill, 2001). Policies of the United States act in concert to support the security of Central Asia to avoid "Afghanicization"; however, the viability and stability of nation-states within the region also require significant economic and political development (Hill, 2001). International powers could further support this development by developing national capacities and resources for providing quality education to support the nascent democracy and economy within the KR and avert governmental and social disintegration (United Nations Educational, Scientific and Cultural Organization, 2011).

The KR remains in an operational survival mode from multiple perspectives and struggles to provide basic services and safeguards for its young and rapidly expanding 
population (Adams, 2000; International Crisis Group, 2016). The bureaucratic vestiges of communism and socialism, the predominance of corruption, and the general lack of personal or professional opportunity make life within this fragile nation extremely difficult (Everett-Heath, 2003; International Crisis Group, 2016). With increasingly severe economic fluctuations and deteriorating social supports, over $30 \%$ of the national population faces systemic poverty (Adams, 2000). In addition, state weaknesses and lack of trust in the governing regime have impacted citizenship in the KR with residents being increasingly reluctant to pay taxes, observe rule of law, or serve in the army (Ruget \& Usmanalieva, 2007). Commensurate rises in violent protest activities, divisive subnational identities, and rates of emigration, particularly among the affluent, educated, and younger generations pose further threats to the national foundation (Ruget \& Usmanalieva, 2007).

Critically, the national education system, itself a legacy of Soviet social development policy, is struggling to provide basic quality education to the KR's youth (Asia Development Bank, 2015; Gita, 2016; Nessipbayeva \& Dalayeva, 2013). International assessments have demonstrated that students in the KR are failing to develop academic skills; the Program for International Student Assessment (PISA) consistently indicated that students lacked basic competencies in science, reading, and mathematics, ranking last in the world (Sainazarov \& Shamatov, 2010). The PISA results also reflected widely varying levels of academic preparedness among students (Sainazarov \& Shamatov, 2010). I observed the long-term consequences of inadequate primary education on higher education as the university struggled to accommodate students with a wide range of preparedness. I posit that the degree and variability of the 
extant educational problems in KR higher education must be contextualized within its near universal access to post-secondary education.

\section{Problem Statement}

The long-term consequences of low academic performance and educational quality have the potential to hinder the economic development and social advancement of the developing KR (Organisation for Economic Co-operation and Development, 2010). The cycle of substandard teaching and learning could potentially be interrupted by instituting effective, practical, and sustainable means for advancing universal student performance and instructional quality in higher education (McCormick, Kinzie, \& Gonyea, 2013). Schön identified complexity, uncertainty, instability, uniqueness, and value conflicts as contributors to ineffective practices and unintended outcomes (1983). The reality of the KR embodies these obstacles to instituting reform and promoting quality educational praxis and explains, in part, the failure of higher education reforms in the country (DeYoung, 2004; Drummond \& DeYoung, 2003; Gita, 2016; Reeves, 2004; Smolentseva, 2017). That most reforms stem directly from external donor agencies and foreign governments also partially explains failures to evince meaningful, positive change (Amsler, 2008; Bayalieva-Jailobaeva, 2017; Tabulawa, 2003). Significantly, the failure of externally developed educational interventions to improve the quality of higher education in the KR poses a significant problem to the developing nation (DeYoung, 2011; Drummond \& DeYoung, 2003). This predicament warrants further investigation into post-Soviet higher education praxis and experiences and oriented the development of the research protocol (see Appendix A). 
Education reform for student success and state stability. The KR has hosted disjointed and fragmented efforts to reform education and improve academic quality, introducing multiple initiatives but generating little measurable progress (DeYoung, 2005). The national government along with public and private educational institutions have been amiable toward reform efforts by donor organizations including the United Nations, the United States Agency for International Development (USAID), the Soros Foundation, and the Aga Khan Foundation (Tabulawa, 2003). However, such efforts have limited sustainability without internal financial, logistical, and professional support. Funding and resources, such as teachers' salaries, in this region are inconsistently provided and fall well below the level required for basic subsistence (Tate, Shamatov, \& Weeks-Earp, 2011). Amid reform efforts, classrooms and educational facilities continue to deteriorate, materials are lacking or absent, corruption is rampant, and a growing generation of students is entering adulthood with limited skills and minimal opportunity for development (International Crisis Group, 2016; Mertaugh, 2004).

Disenchanted youth, disenfranchised ethnic groups, and regional economic and political challenges have significantly destabilized the Kyrgyz nation-state (McGlinchey, 2011; National Council for Sustainable Development of the Kyrgyz Republic, 2012; Ruget \& Usmanalieva, 2007). As recently as 2010, deteriorating economic and social conditions in the South of the country gave rise to ethnic violence between nationals of Kyrgyz and Uzbek heritage and resulted in three days of mass killing, arson, and looting, which the government struggled to contain (Megoran, 2013). Education reform is needed to improve academic quality and rates of student success, but it is also imperative to the security and stability of the developing nation (Everett-Heath, 2003). The KR has a 
better chance of achieving sustainable national development and economic growth if it improves student learning and instruction, and invests in better education nationally (Hanushek \& Woessmann, 2007; Organisation for Economic Co-operation and Development, 2010). However, the literature is sparse on many of the underlying assumptions, educational constructs, and justifications for implementing education reform in a post-Soviet setting (DeYoung, 2005). Developing strategic interventions, much less transforming a national education system in a unique context, requires a theoretical focus to guide the development, implementation, and evaluation of organizational learning and change (Argyris, 1976; Argyris \& Schön, 1974).

The potential of student engagement and reflective analysis. Student engagement is a central aspect of Western higher education policy, practice, and research; various researchers cite the construct as being essential to institutional quality and student success (Kuh, Kinzie, Buckley, Bridges, \& Hayek, 2011, 2006; McCormick, Kinzie, \& Gonyea, 2013). The literature defines and operationalizes student engagement in various ways, but its relevance to academic success has been thoroughly analyzed (Kuh, Kinzie, Buckley, Bridges, \& Hayek, 2011). The most common conceptual definition of student engagement uses a behavioral perspective: the construct is defined by student responses to educationally effective practices and activities, as well as by institutional initiatives and resources (Kahu, 2013; Kuh, 2009).

The National Survey of Student Engagement (NSSE) developed as an assessment for the student engagement construct and a proxy for academic quality as measured by student self-reports of benchmarked effective educational practices (Kuh, 2005). As such, international institutions have applied and interpreted the construct and its 
associated instruments as proxies for quality in post-secondary education (McCormick et al., 2013). Some researchers argue that institutional assessments of student engagement inherently hold potential for reform in education with regard to quality assurance and improvement (McCormick et al., 2013). While the construct was developed within the context of a traditional four-year higher education experience in the US, it has been adapted as an evaluative tool within multiple international higher education systems (Coates \& McCormick, 2014). Several large-scale studies have simply taken NSSE instruments and administered them within various higher education contexts (Chang, 2012; Coates \& McCormick, 2014). However, these analyses skipped the ground-level cognitive work necessary to validate the application of research-based theory in context and effectively influence practice (Schön, 1983; Trowler \& Trowler, 2010). It would have been particularly challenging to directly administer NSSE instruments at the research site as the concept has yet to be explored in a post-Soviet context, and practitioners and students possess widely varying English, Kyrgyz, and Russian language competencies, rendering instrument translation ineffective (Aminov et al., 2010; Orusbaev, Mustajoki, \& Protassova, 2008).

To evaluate the student engagement construct's potential at the research site and gain a greater understanding of the teaching and learning environment within the university, I focused on the five NSSE benchmarks of effective educational practice: 1) level of academic challenge, 2) active and collaborative learning, 3) enriching educational experiences, 4) student-faculty interaction, and 5) supportive campus environment ("NSSE Benchmarks," 2000). Although the benchmarks have since been expanded to ten student engagement indicators and practices, the original five were 
sufficient for the exploratory nature of the study and foreseeable linguistic limitations (“NSSE Engagement Indicators and High-Impact Practices,” 2015). The student engagement construct and the NSSE benchmarks themselves face multiple critiques, which I explore in detail in the literature review (Baron \& Corbin, 2012; Coates \& McCormick, 2014; Zepke, 2014). I nonetheless selected these practices for several reasons: the construct is used as a proxy measure of higher education quality (Coates, 2005; McCormick et al., 2013), and the implementation of evidence-based practices in this post-Soviet context by external organizations is common-practice (Amsler, 2008; de la Sablonnière, Taylor, \& Sadykova, 2009; DeYoung, 2005). In addition, several international systems of higher education have incorporated student engagement into quality assurance and improvement efforts, institutional research, and performancedependent funding mechanisms (Coates \& McCormick, 2014).

Student engagement is a largely unknown if not misunderstood concept at the research site (Participant 01, personal communication, November 15, 2017). Soviet instructional methods and institutional practices are actively employed at the research site and throughout the region, with plausible impacts on student outcomes (DeYoung, 2011). Soviet educational practices include passive one-way student learning, a transactional epistemology (the faculty transfers information to learners), emphasis on lecture and rote memorization in pedagogy, strict discipline, and absolute deference to elders and faculty (Kline, 1957; Shamatov, 2006). Traditional Soviet institutional practices were defined by bureaucracy, complex administration and governance, external and internal top-down leadership, reactive and protectionist supervision, and an emphasis on status maintenance above change or growth (Kline, 1957; Shamatov, 2006). Critically, the Soviet practice of 
graduating all admitted students, in some cases regardless of ability, achievement, or effort persists in the region despite major consequences (Avis, 1990; Kline, 1957).

In this study, I applied reflective analysis techniques within qualitative field research procedures to garner practitioner and student perspectives that shed light on their professional experiences and practices in higher education. Interview protocol items were developed to draw directly from the theoretical framework (see Appendices B and C) and detailed semi-structured interview protocols incorporated reflective lines of questioning (see Appendix D) oriented around the NSSE benchmarks (see Appendix E). While reflective practice has several components, for this dissertation, participants engaged in reflection-on-action using their own experiences and practices to gauge how higher education in the KR can and should be changed (Schön, 1983). Additional practical evidence was obtained through classroom observation using an adapted instrument focusing on visual identifiers of student engagement in higher education practice (see Appendix F). The analysis of evidence and perceptions generated via reflective practice provides ground-level cognitive work that will be foundational for future applications of evidence-based practice in a context such as the KR that embodies complexity, instability, and uncertainty.

Research question and sub-questions. This study focuses on practitioner and student perceptions of Western higher education relative to post-Soviet higher education experiences with emphasis on the educational practices and student/faculty behaviors of NSSE. The primary research question is: How do post-Soviet higher education practitioners reflect on their professional experiences and Western benchmarks of 
effective educational practice? Three sub-questions approach different facets of the research question and orient the analysis around the NSSE benchmarks in this context. SQ1: How are practitioner and student higher education experiences described? SQ2: How are effective educational practices among faculty and students defined? SQ3: How are the NSSE benchmarks and their practical potential interpreted? To strengthen ties to the theoretical framework discussed in the next section, the third sub-question is further defined to analyze each benchmark and their cumulative potential. SQ3A: How challenging is this academic program?

SQ3B: To what extent is learning active and collaborative?

SQ3C: In what ways do practitioners and students interact?

SQ3D: To what degree is the campus environment supportive?

SQ3F: How might the NSSE benchmarks impact praxis and quality?

\section{Conceptual Framework}

The conceptual basis of this dissertation is based on the components underlying evidence-based practice. While this could be construed as a positivist framework, I argue that the realities of practice at the site of implementation warrant an interpretivist approach employing humanistic qualitative research methods. The study builds upon a postulation that the relationship between empirically-based evidence and functional practice harbors additional layers of complexity, partially explaining unintended outcomes and consequences of best practices (Schön, 1983). Understanding the differences between theory-driven practice in its original context and at an implementation site comprises the basis of this study with the mitigation of negative outcomes being a key target. The disconnects between evidence-based practices 
developed from research and driven by theory in one theoretical context and actual practice and outcomes as implemented in another practical context are explored through reflective analysis in this study (see Figure 1).

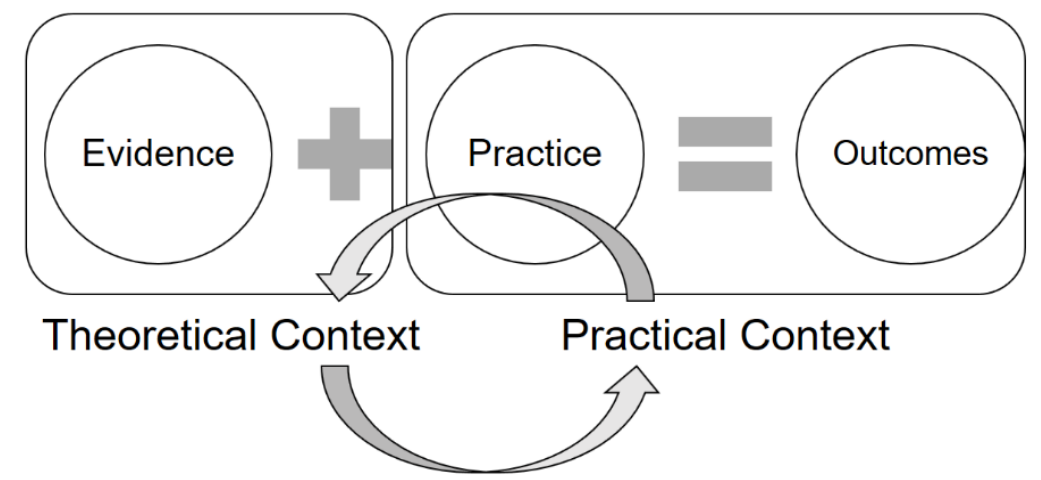

Figure 1. Conceptualization of Evidence-Based Practice Across Theoretical and Practical Contexts. This figure depicts the respective contexts inherent to evidence and practice as well as outcomes. Specifically, student engagement theory informed by higher education research resulted in the development of evidence-based practice and the NSSE benchmarks from within a traditional, Western higher education experience. In application, this can be conceptualized as a developmental process wherein higher education outcomes are driven by practice as justified by evidence-based practice derived from student engagement theory (see Figure 2). 


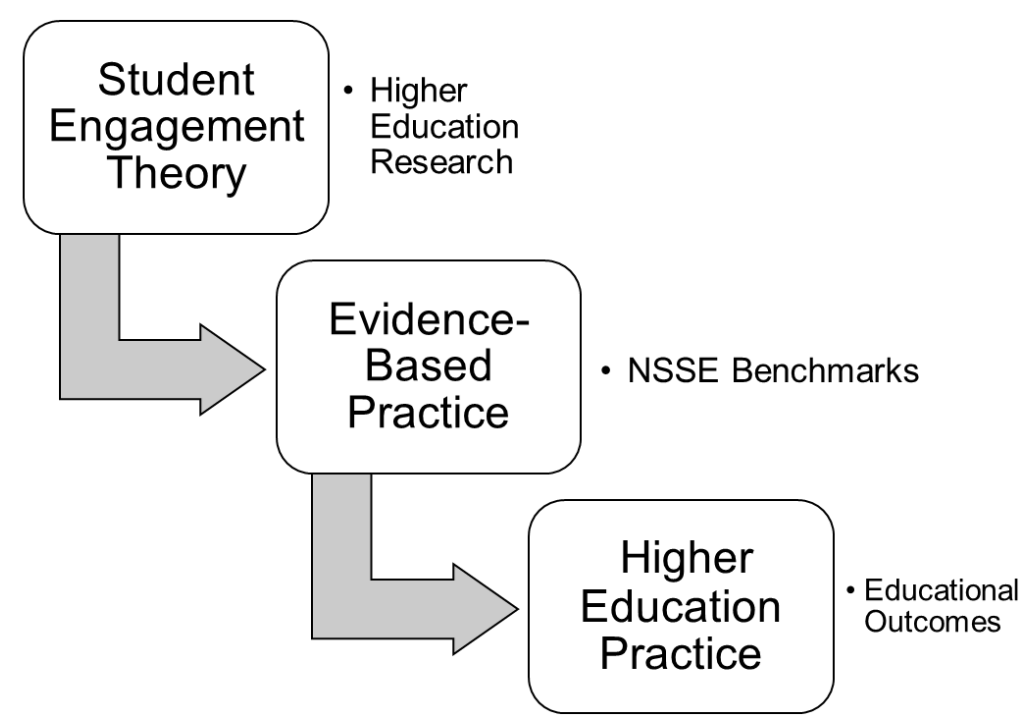

Figure 2. Theoretical Basis of Higher Education Evidence-Based Practice. This figure depicts the student engagement theory which guides higher education research to inform evidence-based practice as defined by the NSSE benchmarks and effect change to higher education practice and educational outcomes.

The perceptions of these benchmarks to change post-Soviet higher education practice and potentially yield positive educational outcomes comprise the reflective focal point of the analysis.

\section{Theoretical Framework}

This study analyzes participant higher education experiences and perceptions of Western-based practices associated with student engagement using the NSSE benchmarks as a theoretical framework. The benchmarks reduce the scope of the study by orienting it around tenets of student engagement theory, specifically empirically-based practices and experiences associated with the concept and positive student outcomes.

This framework guides the study by focusing on higher education experiences and effective practices, as well as perceptions of the NSSE benchmarks. 
Within the rapidly transitioning context of the KR, universities have had to adapt to a variety of practical realities that impact student outcomes. The university and the program under analysis must embrace change and continuous improvement to prevent becoming wholly irrelevant to students and survive in its context. Cultivating reflective analysis and continuous improvement is a developmental process, and as such this study serves as an extended pilot for such means of organizational development. This analysis is oriented around critical reflection on higher education experiences and practices.

Practitioners and students are placed at the center of the analysis and reflect on their own university experiences relative to Western practices. The data collection process strengthens the study by coordinating additional sources of evidence pertaining to the post-Soviet higher education experience, specifically via student inclusion. The analysis and conclusion portions of this study are similarly strengthened by maintaining my positionality as an external practitioner and researcher, and developing reflections on my own praxis, post-Soviet practices, and Western practices. Reflection on my positioning within the context of the research site accounts for intrinsic assumptions and biases while contributing to the quasi-comparative nature of the study.

This study distills down to the following: the NSSE benchmarks are applied as a theoretical guide for practitioners and students at the site to reflect on their teaching and learning experiences and higher education practices at the research site.

\section{Operational Definitions}

Within the context of this study, the following definitions apply: 
Administrators: senior faculty who have assumed leadership positions within the program under analysis, most of whom have several years of experience and hold the equivalent of a $\mathrm{PhD} / \mathrm{EdD}$ or Doktor Nauk.

Benchmarks: five sets of empirically supported, Western practices that are measured by the College Student Report for NSSE and used as proxies for student engagement and academic quality (see Appendix E).

Case: the learning and teaching environment within a foreign language teacherpreparation program at a regional public university in which the embedded units of analysis are practitioners and students (see Figure 3).

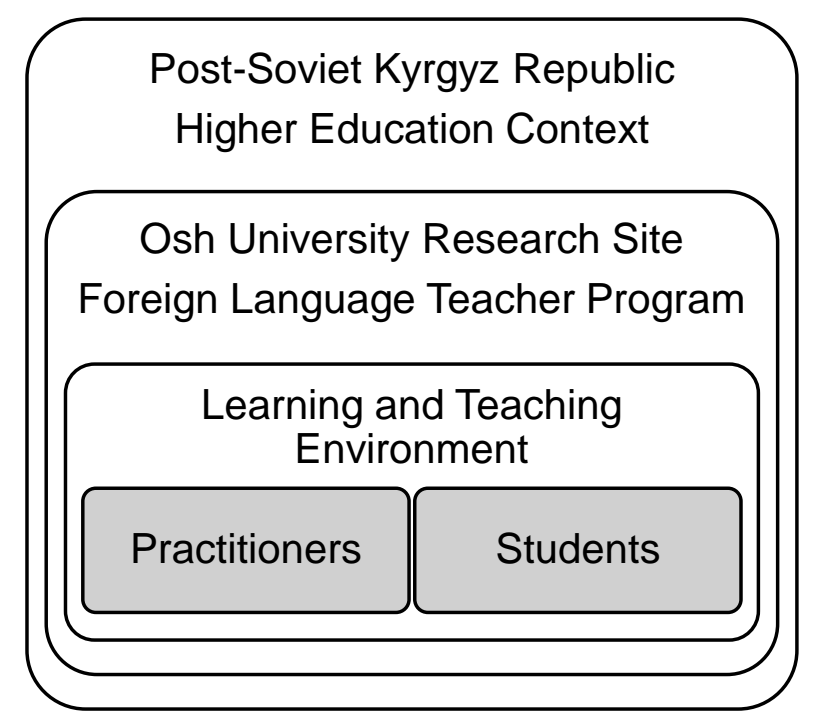

Figure 3. Research Site Learning and Teaching Environment Ethnographic Case. This figure depicts the embedded units of analysis: practitioners and students within the program learning and teaching environment case.

Ethnicity: a shared cultural, genetic, or geographical heritage that defines a group of people.

Faculty: instructors within the case, most of whom hold the equivalent of a master's degree or Kandidat Nauk. 
Nationality: the legal status of an individual or group relative to a geographic location or nation.

Participants: all individuals contributing to the data collection stages of this study.

Practice: the official actions an individual should conduct within their designated role.

Practitioners: administrative and educational professionals at the research site.

Praxis: habitual or established practice; practical application in context.

Professional Capacity: a cumulative measure of technical capacity, skill, expertise, and experience within a profession.

Program: a four-year undergraduate teacher-preparation program that focuses on English as a Foreign Language (EFL) and confers a national teaching credential.

Proposition: a statement expressing a pre-existing judgment or opinion.

Research Site: a regional university located in Osh, a historic city of approximately 250,000 people located in the Ferghana Valley in the south of the KR (see Figure 4).

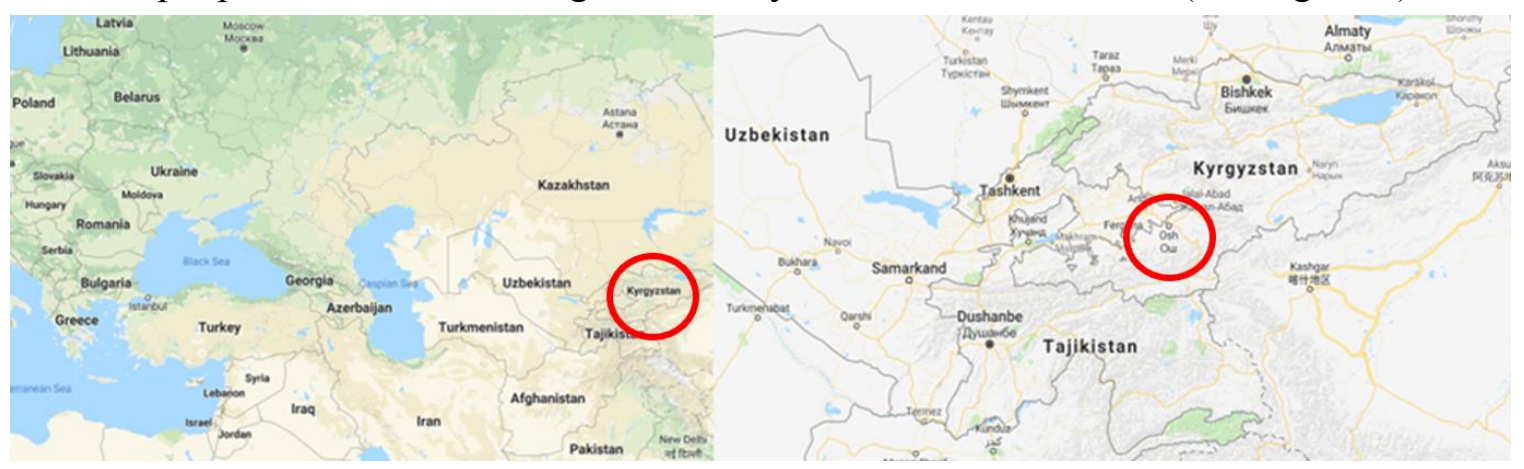

Figure 4. Kyrgyz Republic Research Site Map. Left: Map of Central Asia, the Middle East, and Eastern Europe. This figure provides geographical context, Kyrgyzstan is circled for emphasis. Right: Map of Osh, Kyrgyzstan. This figure highlights the location of the research site within the Ferghana Valley, Osh is circled for clarity (Google, 2017.). 
Stakeholders: administrators, faculty, and students who are impacted by the case, as well as families, parents, students, and community members who are external to the case. Student Engagement: an organizing construct for institutional assessment, accountability, and improvement efforts, encompassing the time and effort students spend on activities that correlate with positive outcomes, and institutional support for such participation (Kuh, 2009).

\section{Study Design and Research Methods}

This study employs an ethnographic case study design and qualitative field research methods as defined by Creswell (2008) and detailed in the research protocol submitted for Institutional Review Board approval (see Appendix A). This design is appropriate given the critical nature of the extent of educational quality decline in the $\mathrm{KR}$, and as the post-Soviet higher education context presents unusual circumstances. The theoretical framework of the study - the NSSE benchmarks of effective educational practice - dovetails nicely with the contextual nature and practical focus of the ethnographic case study design. This study also developed as a test for the theoretical propositions underlying the NSSE benchmarks as detailed by Kuh (2002): 1) what students do during university matters more than who they are or where they attend; 2) good practices yield student engagement; 3 ) institutions with high student engagement can claim to espouse greater academic quality; and 4) measures of student engagement have practical potential within higher education reform.

Data collection focuses on interviews with practitioners, consisting of faculty and administrators, and students within a foreign language teacher preparation program at the research site. I selected the university in Osh as the research site because I had, since 
completing my fellowship, maintained both access to the site and positive professional relationships with program stakeholders. I employ detailed interview protocols and classroom observation instruments to capture multiple qualitative sources of evidence to compensate for the lack of accurate and reliable quantitative data found throughout the research site and region (see Appendices D and E). Given the developing context of the research site, I apply qualitative field research methods to remain amenable to unexpected changes at the research site - a tenet of adaptive design ingrained within traditional case study research (Yin, 2017). Chapter Three details the specifics of the study design, the data collection and analysis procedures, and discusses considerations for analytical rigor, threats to validity, and contextual constraints.

\section{Assumptions, Limitations, and Delimitations}

This section presents the assumptions, limitations, and delimitations of the study as defined by Simon and Goes (2013) within the scope of the ethnographic case.

Assumptions. The following assumptions guide this study:

1. Practitioners and participants can be guided through the process of reflecting on their higher education experiences and instructional praxis.

2. Western-based practices have some relevance within the case relative to praxis.

3. Participants can comprehend and interpret the language and content of Westernbased benchmarked practices.

4. Participants feel comfortable voicing critical and genuine opinions of the program, their experiences, and the current state of the research site. 
5. Contextual factors related to complexity, instability, and uncertainty will not significantly affect the data collection and analysis processes or jeopardize study integrity.

6. Triangulating qualitative sources of data and performing member checks will contribute analytical rigor to the analysis.

7. The ethnographic case study design and qualitative methodological approach will reveal practitioner perceptions of the practical potential of the benchmarks.

Limitations. The following limitations are observed in this study:

1. The ethnographic case study design restricts the generalizability of findings.

2. The university leadership restricts access to data, documents, and has significant influence on participants.

3. Participants possess highly variable English language abilities which impacts the reliability and quality of findings.

4. Extensive corruption, cultural differences, and language barriers in the case impact and influence participation and responses.

5. Quantitative data, assessments, and measures are inaccessible and unreliable thus they are not included in the study.

Delimitations. The following delimitations are observed in this study:

1. The study explores practitioner and student higher education experiences and perceptions of Western evidence-based practices as potential means for improving educational quality at the research site. 
a. I selected the benchmarks, rather than the NSSE itself, as the analytical focus given the complexity, expense, and general infeasibility of full instrument translation and the potential limited applicability of findings.

b. A conceptual exploration of the engagement construct would not have been directly relevant and might have lacked practical application, which was valuable to participants, stakeholders, and general study relevance.

2. I restrict the scope of the analysis to a single post-secondary foreign language teacher preparation program in southern KR.

a. Phenomenological and grounded theory qualitative methodologies would have limited the application of relevant theory, contributing greater complexity and length to the study without a justifiable rationale.

b. The adaptive nature of ethnography and qualitative field research methods compensate for the changeable nature of the research site.

\section{Relating the Discussion to Audiences}

This qualitative study offers the most potential benefit to program stakeholders and study participants, but it also holds implications for education reform scalability and sustainability in developing contexts (Robinson \& Winthrop, 2016). Student engagement, and its applications, present tremendous potential for change through evidence-based practice (McCormick et al., 2013). However, the underlying justification for implementing such action must be rooted in sound evidential and theoretical bases, and substantiated by ground-level cognitive work (Schön, 1983; Trowler \& Trowler, 2010). International donor organizations, educational consortia and foundations, and numerous governmental entities make sizable investments to improve student learning 
often with mixed returns (DeYoung, 2004; Freedberg, 2017). By empowering participants as reflective practitioners, this study adds additional perspective to education reform scaling, maintenance, and implementation, thereby supporting returns on investment. Empirically supported, thoroughly financed, and well-intentioned reform efforts in the United States have produced unintended outcomes, failing to provide equal access to quality education and even exacerbating inequities in some cases (Dauter \& Olivieri, 2017; Gates \& Gates, 2018). By analyzing practitioner perceptions associated with practices associated with the student engagement construct prior to implementation, buy-in among impacted stakeholders who value contextual and practical validity will be fostered, and such support advances the potential for evincing positive intended returns on desired outcomes.

\section{Contributions to Theory and Practice}

This study contributes to the body of higher education research by focusing on an institution in the developing post-Soviet context of Central Asia, a region that has traditionally lacked awareness among Western audiences and researchers. The analytical focus of this ethnographic case study is supported through the evaluation of perceptions of student engagement associated practices comprising the NSSE benchmarks. I investigate the student engagement construct's practical potential in a developing and rapidly changing higher education environment. While this study did not actually implement the benchmarked practices, findings could provide direction for future action research.

Higher education researchers have called for increasingly nuanced investigations of the student engagement construct specifically incorporating qualitative evidence 
(Kahu, 2013; Zepke, 2014). Krause and Coates promoted a broader understanding of student engagement as a process with multiple dimensions, developed this definition via quantitative and qualitative methods, and assessed whether measures, scales, and factors hold true in international contexts (2008). Similarly, Kuh cautioned about the interpretation and use of student engagement data, arguing that practitioners must proceed judiciously to identify the best forms of engagement for each circumstance and different groups of students (Kuh, 2009a). By exploring the perceptions of student engagement within this research site, I analyze its practical potential within a complex, uncertain, and unstable higher education context. In addition, I evaluate its perceived potential for promoting positive change in post-Soviet teaching and learning environments. The findings of this study contribute to the body of evidence substantiating the application of evidence-based practices associated with student engagement and their efficacy. In this dissertation, I present ground-level evidence essential for potential practical education reform in post-Soviet higher education contexts. It is my intent that the evidence obtained from this study will assist in guiding future effective educational intervention strategy development.

\section{Summary}

Higher education research lacks evidence investigating practitioner and student perceptions of Western student engagement practices in developing and post-Soviet contexts. Conversely, there is an abundance of literature supporting the assessment and importance of student engagement, but a dearth of research into practitioner and stakeholder perceptions of the construct's associated practices relative to existing educational praxis. The literature is mute on the construct's practical potential within a 
post-Soviet higher education environment, such as that of the institution under analysis, providing further justification for the present study. Chapter Two presents a review of the pre-Soviet, Soviet, and post-Soviet contexts comprising the modern KR, details the existing higher education literature on student engagement, and provides further analysis of the theoretical framework underpinning this study. 


\section{CHAPTER II}

\section{LITERATURE REVIEW}

This study developed to bridge a gap in higher education literature regarding the perceived potential of Western evidence-based practice to address problems in postSoviet higher education. As the ethnographic case study centered on a foreign language teacher preparation program in the Kyrgyz Republic (KR), this literature review begins by providing an overview of the history of the nation and its people over three distinct phases: pre-Soviet, Soviet, and post-Soviet. Focus is placed within each era on higher education and educational reform to limit the scope of the review. The discussion then concentrates on the student success literature from which the student engagement construct flows. This content highlights the sociocultural conceptual framework of the construct developed by Kahu, the inclusive conceptualization of the student engagement construct focused upon throughout this study (2013). Finally, the NSSE benchmarked practices used as the theoretical framework are discussed with emphasis upon their alignment with the development and execution of this dissertation. The NSSE benchmarks are detailed then followed by commentary on evidence-based educational practice and the tenets of the reflective practitioner model applied within data collection and analysis (Schön, 1983). A summary then indicates the core components of the review, providing orientation as to the analytical means and significance of the study within higher education research and practice. 


\section{Pre-Soviet Central Asia}

While a comprehensive analysis of the civilizations preceding the modern-day KR is beyond the scope of this literature review, a brief overview of the historical background of the region is presented to situate the study within its current context. To discuss pre-Soviet Kyrgyzstan would ignore the reality that the borders of the modern KR were created by decree under Soviet rule along with the typologies of ethnicities making up the indigenous populations and existing national identities (Landau \& KellnerHeinkele, 2011; Silova, 2011). The pre-colonial legacy of the Ferghana Valley region comprises portions of modern Uzbekistan, Tajikistan, and the southern KR (Starr, 2011). The history of this region spans millennia and encompasses a diversity of cultures and ethnicities who came into contact via long-established trade routes and the interaction of agrarian farmers and nomadic tribes (Saidov, Anarbaev, \& Goriyacheva, 2011; Saidov et al., 2011). Osh, the de facto capital of modern southern KR, was a strategic point on the Great Silk Road what with Sulaiman-Too mountain forming the original culturalideological center for various cultures, faiths, and kingdoms (Saidov et al., 2011). Multiple conquerors including Timur or Tamerlane and Babur, who have assumed legendary status in modern KR, competed with Arab, Turkic, and Mongol forces to maintain control of the Ferghana resulting in a nexus of cultures, languages, people and religions (Saidov et al., 2011). The interaction of civilizations with widely divergent beliefs and practices, and the complexity and consequences of such interaction defines the Ferghana Valley (Starr, 2011). Conflicts and consequences provided the historical conditions leading to the development of the modern nation-states comprising Central Asia (Khazanov, 1995). 
The challenge of reconstructing history in this region is significant as many of the ancient civilizations within the region lacked written language (Tchoroev, 2002). Reconstruction historiography would eventually become essential in the post-Soviet world as newly formed nation-states sought to legitimize and substantiate their ethnicities and nationalities from their dictated identities (Kubicek, 1997; Tchoroev, 2002). Most historical reconstructions developed by proxy using external perspectives from Arabia, China, Persia, and Russia as indigenous accounts were either not written or maintained (Saidov et al., 2011; Tchoroev, 2002). All state archives for the various Central Asian khanates, political organizations ruled by a khan, including the Kokand Khanate, a feudal state in existence from 1709 to $1876 \mathrm{CE}$, were destroyed after the departure of its last ruler (Dubovitskii \& Bababekov, 2011). The loss of this information essentially erased the history of human civilization at the intersection of the Muslim khanate, the Orthodox Christian Russian empire, and the Buddhist-Confucian China of the Qin Empire (Dubovitskii \& Bababekov, 2011).

The influence of Russia, whether Tsarist, Soviet, or post-Soviet is an absolute throughout this region and historical accounts reflect the progression of Russian policies and their impacts on the people of Central Asia. Tsarist Russia violently overtook the Kokand Khanate in 1876 and continued to expand the Russian Empire throughout the region (Dubovitskii \& Bababekov, 2011). This expansion provoked Anglo-Russian rivalries and resulted in numerous uprisings as the estimated five million indigenous people sought to maintain some degree of control and independence (Dubovitskii \& Bababekov, 2011). The Russian Empire conquered the khanates on a primarily economic rationale: to obtain resources, increase access to markets, and control regional trade 
routes; as well as a political and military affront to the British who had expanded into Afghanistan and India (R. Abdullaev, Khotamov, \& Kenensariev, 2011). Ethnic Kyrgyz nomads, shepherds, and cattle breeders throughout the Ferghana Valley actively resisted Russian colonization by either retreating to inaccessible mountain areas or through armed rebellions with clans led by Pulat Khan and the female leader Kurmanjan Datka (R. Abdullaev et al., 2011). The colonial goals of tsarism shaped Russian imperial policy within Turkestan, formerly Kokand, by instituting a new system of law, politics, and socioeconomic relations which redefined the region as a periphery and made it completely dependent on the Russian Center (R. Abdullaev et al., 2011).

With the creation of Turkestan, Russia managed to functionally subjugate the Ferghana Valley and the people of Central Asia. This transition established ties of control, deference, and dependence on Russia throughout the region some of which remain in various forms to the modern day. The indigenous ethnic Kyrgyz populations gradually abandoned nomadism and adopted a settled existence of trade and industry within a commodities and money-based economy in direct competition to Slavic colonialists resettled under Russian policy (R. Abdullaev et al., 2011). Tsarist despotism remained in place until the Kerensky and Bolshevik Revolutions of 1917 and throughout this era the indigenous populations of the Ferghana either emigrated, remained and continued to view colonial domination as temporary and actively resisted, or remained and passively resisted while promoting gradual reform under a movement known as Jadidism (R. Abdullaev et al., 2011). Russian policies of domination and subjugation throughout Central Asia achieved the economic and political ends of securing and supporting the tsarist empire through the manipulation of the indigenous populations 
throughout the region. Rather ironically, social, economic, and political instability within Russia itself ultimately resulted in a revolution which fundamentally altered the lives of individuals under the Soviet sphere of influence.

\section{Pre-Soviet Education}

Education within Central Asia prior to tsarist Russia was largely informal and faith-based, reflecting the cultures of the indigenous nomadic tribes and lifestyles found throughout the Ferghana Valley and greater region (M. S. Johnson, 2004). Three educational strategies associated with the Qadimist, Tsarist, and Jadidist movements comprised educational pedagogies, attempts at reform, and curricular content within preSoviet Central Asia (M. S. Johnson, 2004). Education preceding Russian colonialism was primarily centered around Islamic faith with Muslim madrassas, maktabs, and qarihanas; rather interestingly six Jewish schools were also noted in school statistics dating from 1875 (Dubovitskii \& Bababekov, 2011). Traditional Qadimist Islamic education emphasized reading of the Qur'an in addition to formal Arabic study, some larger urban area schools had a full range of curricular subject areas and emphasized religious studies in addition to apprenticeships and vocational training (M. S. Johnson, 2004). Qadim schools had certain drawbacks including the lack of coeducation beyond the primary level, a rapid decline in academic quality, and little to no literacy and writing skill development, especially in native languages (M. S. Johnson, 2004). While Qadimist education failed to educate all individuals, particularly women, to the extent needed to participate in the international sphere, within the confines of a nomadic and tribal culture, it arguably served its purpose in providing cultural and religious education (M. S. Johnson, 2004). 
With growing Russian influence and predominance within indigenous civilizations and cultures, the limitations of Qadimist education began to have larger impacts, particularly after the dissolution of the khanates. In line with Russian policy, education was used to inculcate and indoctrinate indigenous populations with thoroughly Russian beliefs and practices for the benefit of the empire (M. S. Johnson, 2004). Tsarist education incorporated elements of socialization into education by using indigenous teachers and language to force the adoption of the Cyrillic alphabet and an intensely conservative Russian patriotism (M. S. Johnson, 2004). A chronic lack of funding, maintenance of archaic curricula, and outright ethnic discrimination that purposefully benefited Slavic colonial settlers over native student populations limited the efficacy of tsarism (M. S. Johnson, 2004). That indigenous populations objected to the imposition of Russian education should come as no surprise as Tsarist schools essentially functioned to reinforce Russian culture and political interests while denigrating native cultures and education as fundamentally backwards (R. Abdullaev et al., 2011). The systemic problems found within Tsarist education would also foreshadow the issues which scientific management and techno-rational learning failed to address throughout the Soviet empire.

Jadidism came into prominence toward the end of the nineteenth century and encompassed various aspects of intellectualism and the Muslim faith relative to the cultural, political, and social issues at the time (R. Abdullaev et al., 2011). Jadidist leaders had observed that traditional Qadimist religious education was outdated and failed to teach students essential learning skills in the changing environment (R. Abdullaev et al., 2011). They set about developing "New Method" schools incorporating 
the audio-lingual method for reading and writing, new subjects including natural and social sciences, and European facilities and resources within classrooms (R. Abdullaev et al., 2011). Critically, the Jadidists sought to provide child-centered instruction and establish indigenous written languages and create native language primers, precursors to the cultivation of indigenous national identities (Johnson, 2004). These efforts, while incorporating the best of European and Central education thought, were condemned by Russian officials due to their efforts to change the status quo in education by incorporating new ideas and practices and textbooks which had yet to be approved and were deemed to constitute divisive pan-Islamic and pan-Turkic ideas (R. Abdullaev et al., 2011). In trying to find a middle path between emigration and annihilation, Jadidism managed to promote education reform within cultural and social modernization; these achievements ultimately resulted in failure as both Islamic conservatives and Russian officials condemned the movement (M. S. Johnson, 2004).

Throughout Central Asia, education can be interpreted historically as a vehicle for social and political change and a means of cultural preservation. The culture clashes resulting from the imposition of Russian ideologies and authoritarianism were representative of native populations pushing back from mandates for secular and scientific societies. Endemic to the various movements within pre-Soviet education are beliefs and value judgments from leaders and policymakers indicating what education is meant to do and how it is supposed to do it. That educational quality has been an issue in this region since the 1800 s indicates the historical nature of the problem. That competing interests have consistently incorporated agendas and ideologies within education reform 
efforts to theoretically improve academic quality foreshadows issues encountered within contemporary reform.

\section{Soviet Kyrgyzstan}

Destabilization within tsarist Russia due to internal forces and external events resulted in revolutions across several aspects of life which ultimately redefined Central Asia and the Ferghana Valley. That reform and revolution within Russia had domino effects throughout regions under their sphere of influence was indicative of the extent of power and control leadership in Moscow continued to hold throughout the region. This power and control materialized in different policies, practices, and beliefs with a range of consequences and outcomes. Tracing the historical effects on the development and management of Soviet Kyrgyzstan indicates the conditions preceding the creation of the modern-day KR and explains some of the challenges remaining to this day.

The Kerensky and Bolshevik Revolutions of 1917 ended the tsarist despotism of the Russian empire with the fall of the House of Romanov and uprooted a form of autocratic authoritarian governance in existence for nearly two centuries (Abashin, Abdullaev, Abdullaev, \& Koichiev, 2011). The violent removal of the absolute monarchy further destabilized Central Asia as the provisional Turkestan Autonomous Government quickly devolved as competing forces jockeyed for power in the resulting political vacuum (Abashin et al., 2011). The Ferghana Valley was besieged by open conflicts between the indigenous resistance forces of moderate socialists, nationalist reformers, and conservative Islamists and the Bolsheviks who with the Communist party under Marxist revolutionary leader Lenin had consolidated power under the Union of Soviet Social Republics (USSR) (Abashin et al., 2011). The transition from tsarist Russia 
to Soviet Union can be viewed as being anything but seamless, and the resulting power struggles further jeopardized the already tenuous livelihoods of many people within the region. The adoption of alternative means of governance with vastly different power structures and ideologies required major economic, political, and social adjustments intended to revolutionize life for the masses for the better (Abashin et al., 2011). The gaps between intents and outcomes, theories and practices, and perceptions and realities only widened throughout this period.

With the establishment of the USSR, life for the diverse populations under the Russian sphere of influence was compartmentalized and engineered in an effort to actualize the economic and political ideologies and structures of communism and socialism (Abashin et al., 2011). Bolshevik totalitarian policy advanced strengthening control over peripheral, non-Russian areas including Turkestan by redrawing national and administrative borders along approximate ethnic and national lines to limit the influence of the remaining Muslim elite (Abashin et al., 2011). Crucially, Bolshevik leaders decided which regional populations constituted each ethnicity, consolidating the Uzbeks and Sarts while diving Kara-Kyrgyz and Kara-Kalpaks before assigning each ethnic group to a nationality within borders which they also officially and unequivocally established (Abashin et al., 2011). This process of delineating national borders continued until 1936 when the Kyrgyz Autonomous Region was removed from the Russian Soviet Federated Socialist Republic and became the Kyrgyz Soviet Socialist Republic (KSSR), a largely arbitrary decision from Moscow decreed once again without discussion or input (Abashin et al., 2011; Khazanov, 1995). 
The process of creating and defining the nations comprising the Central Asian Republics (CAR) colloquially referred to as Kazakhstan, Kyrgyzstan, Tajikistan, Turkmenistan and Uzbekistan within the USSR was characterized by several practices of the centralized totalitarian state (Abashin et al., 2011). These practices included a superior Russia colonialist attitude toward the CAR, stringent secularization and socialization of many aspects of life, aggressive Russification of culture and language, and critically the establishment of artificial borders which purposefully divided ethnic groups and tribes (Abashin et al., 2011; DeYoung \& Santos, 2004). Soviet policy over the following three decades shifted from tolerance and expansion to coercion, exploitation, and repression as policies of economic collectivization and religious suppression and established Russification strategies sought to create a singular Soviet identity throughout the region (K. Abdullaev \& Nazarov, 2011). With policy and the planned economy being set by principles of scientific management and technical rationality under absolute order from a far-removed central power, the status of the individuals who made up the newly formed ethnicities, identities, and nationalities within these distant lands echoed of the substantial challenges and conflicts to come.

The Stalin era from 1929-1952 solidified Central Asia as a peripheral region within the USSR purposefully engineered to remain "backward", segmented, and dependent upon Russia for all decision-making, leadership, and economic policy (K. Abdullaev \& Nazarov, 2011). Examples of such engineering include the demarcated borders within the USSR which inhibited communication and transit between republics by separating major cities by impassable mountain ranges and railways designed solely for the extraction of raw materials from Central Asia to industrial centers in the Russian 
Soviet Federative Socialist Republic (K. Abdullaev \& Nazarov, 2011). Methods of terror were orchestrated by the Central Committee led by Stalin to advance compliance with Communist policy and resulted in dissent being met with active repression and suppression of thought; this also spurred the creation of the Main Administration of Collective Labor Camps (GULAG) and ultimately resulted in the deaths of millions throughout the USSR (K. Abdullaev \& Nazarov, 2011). The draconian tyranny of Soviet policy, especially under Stalin, can be viewed as having Orwellian characteristics with control being maintained through propaganda, surveillance, misinformation, and manipulation of the past, all tenets of Nineteen Eighty-Four (Hitchens, 2008). That this was the lived reality for many in the Soviet Union is sobering given the brutality and violence which ended up resulting from a social revolution theoretically intended to benefit.

Under the leadership of Khrushchev and Brezhnev, Kyrgyzstan experienced its most stable era of accelerated economic modernization and prosperity under Soviet rule with industrial development in factories throughout republic, agrarian advancements in mechanization, and the establishment of social welfare support and protections (R. Nazarov \& Shozimov, 2011). While economic and social modernization succeeded to varying degrees, the creation of a unified Soviet identity throughout the Ferghana Valley primarily failed with ethnic identities and traditional cultural practices remaining steadfast and pushback to Russification and Sovietization strengthening (Madaminzhanova \& Mukhtarov, 2011). The Soviet invasion of Afghanistan in 1979 had the unintended consequence of reactivating radical religious networks throughout Central Asia and sparked re-Islamization just as the economic and political reforms of perestroika 
and glasnost began to significantly impact the livelihoods of many throughout the KSSR (Shozimov, Beshimov, \& Yunusova, 2011).

Questions surrounding national identity fueled Kyrgyz-Uzbek conflict and contributed to the Osh Events of 1990 and subsequent ethnic conflicts where two ambiguously defined ethnicities who had cohabitated for centuries in the Ferghana valley resorted to violence on the basis of preserving their respective identities regardless of geography (Shozimov et al., 2011). Soviet governance and policy regarding such conflicts and frequent border disputes and changes is best summarized in a decree by the Twenty-Second Party Congress in 1962 which stated:

In the Soviet republics, people of many nationalities live and work together. Borders between union republics within the borders of the USSR increasingly lose their importance; as all nations are equal, their life is built on a single socialist foundation. (R. Nazarov \& Shozimov, 2011, p. 144).

It is relatively easy to see how conflict, discontent, and unrest might result among individuals who have been historically marginalized and placed into arbitrarily assigned and defined ethnicities and nationalities. Faced with declining living conditions and specifically restricted from any semblance of control over their own lives and livelihoods, conditions for civil unrest were thoroughly established.

The collapse of the Soviet Union in 1991 resulted in independence being thrust upon Kyrgyzstan and the effective abandonment of Central Asia and the nations making up the Ferghana Valley (Beshimov, Shozimov, \& Bakhadyrov, 2011). The now independent Russian Federation set about aggressive domestic policies of democratization, liberalization, stabilization, and privatization to transition to an open, market-based economy while emphasizing Russian interests and sovereignty (Khazanov, 1995). The formation of the CIS, a loose confederation of the former Soviet republics, 
was a largely symbolic organization as multiple conflicts between newly independent republics broke out and regional economic and social conditions continued to decline (Khazanov, 1995). It is important to note that in a 1991 referendum, 95\% of Kyrgyz voters favored the preservation of the Soviet Union. If this figure is accurate and representative of the Kyrgyz population, it depicts an unwanted independence particularly in the aftermath of the Osh Events (Beshimov et al., 2011). Clearly, these cultures and civilizations - unfamiliar with the concept of the nation-state and unprepared for the realities of a developing democracy and free market economy_faced an uphill battle as they tried to establish governments and maintain social support (Beshimov et al., 2011). The transition from Tsarist colony to Soviet republic defined and divided the Kyrgyz people and their role within the planned economy; the demise of the USSR left economic, political, and sociocultural vacuums throughout.

\section{Soviet Education}

The Soviet-style education system instituted throughout the CAR produced many positive outcomes: near universal literacy, relative equity of access and opportunity, and the concept of free, secular coeducation for all (M. S. Johnson, 2004). On the other hand, Russian colonialism and Soviet socialism contributed to less-than-desirable attributes: bureaucratic management systems, outdated ideological and political curricula, narrow emphases on vocational education, and integration into centralized economic planning (Avis, 1990; M. S. Johnson, 2004; Kline, 1957). The very creation of the educational system was fraught with challenges as Soviet leadership dictated quasi-affirmative action policies to expand formal education and indigenization, hoping to cultivate indigenous elites (M. S. Johnson, 2004). Soviet regimes offered inconsistent educational objectives, 
leading to unintended consequences; for example, one regime ordered that indigenous languages be codified into the Latin alphabet and taught this way in schools, but then decided to favor Cyrillic script over Latin, and finally replaced the whole initiative with a mandatory monolingual Russian policy (M. S. Johnson, 2004; Landau \& KellnerHeinkele, 2011). Similarly, tolerance for Islamic education was replaced with hostility, aggressively scientific atheism, and secularization, which transitioned to the outright suppression of Islamic educational values and practices (Johnson, 2004).

Soviet education demanded high standards of achievement, prosocial behavior, and socialized practices; very young students participated in group activities that emphasized cooperation, respect for the group's interest over one's own, self-regulation, and deference to adults and superiors (Elliott \& Tudge, 2007a). Western commentators quickly noticed the strong ideological component of Soviet pedagogy, which limited debate, controversy, and individuality, particularly given the inflexibly authoritarian teaching methods (Elliott \& Tudge, 2007a). Markowitz (2000) noted that students who lacked interest or motivation in the Soviet classroom were apt to withdraw rather than disrupt class, resulting in greater student passivity. Democratic classroom practice typifies the ideological divide between Soviet and Western educational theory along with other Western concepts such as individual agency, personal choice, student interests, and authority versus self-regulation (de la Sablonnière et al., 2009; Elliott \& Tudge, 2007a).

\section{Post-Soviet Kyrgyz Republic}

The collapse of the USSR resulted in tremendous political and social upheaval throughout Central Asia, with consequences and ramifications that are still developing (Everett-Heath, 2003). Kyrgyzstan (officially renamed as the Kyrgyz Republic after 
1993) gained independence by default upon the collapse of the USSR, and the postSoviet socioeconomic realities within this landlocked and resource-scarce country were shocking and significant (Anderson \& Pomfret, 2000; Dudwick, 2003). The post-Soviet transition left the KR in precarious economic, educational, and social positions (International Crisis Group, 2016). Qualitative investigations conducted within the newly-independent KR captured the experiences of poverty and the realities of nationhood (Kuehnast, 2002). Interviewers contextualized the effects of the post-Soviet transition and highlighted the disconnect between survey responses and the objective truths of life and poverty among disenfranchised and vulnerable populations (Kuehnast, 2002).

Today, the KR is situated at a potentially-dangerous nexus of stalled economic growth, persistent social inequality, episodic social instability, and declining human capital that correlates with systemic educational issues (International Crisis Group, 2016). A lack of local industry and an externally-dependent and tenuous national economy further destabilizes the nation (Asian Development Bank, 2017a). The KR's gross domestic product (GDP) is comprised of two primary sources - a single gold mine and international remittances from Kyrgyz nationals working abroad — while membership within the Eurasian Economic Union (EEU) has produced short-term benefits and longterm uncertainty (ADB, 2017a). The "June events" of 2010 evinced the link between economic uncertainty and social instability. The southern ethnic Kyrgyz population clashed with ethnic Uzbek populations, killing hundreds, displacing tens of thousands, and spreading political chaos throughout the region (McGlinchey, 2011). The sociocultural and economic risk factors that precipitated the First and Second Tulip 
Revolutions, civil uprisings taking place throughout 2005 and 2010, and various ethnic conflicts remain to this day, such that political regime change or heightened economic instability could lead to similar bloodshed (The Sentinel Project for Genocide Prevention, 2013). Finally, instability is driving radicalization of the Kyrgyz youth, who are increasingly pursuing membership in fundamentalist terrorist organizations and have already been implicated in several acts of international terrorism (U. Nazarov, 2016; Routray, 2017).

\section{Post-Soviet Education}

The vestiges of the former Soviet Union have dictated the modernization of postSoviet higher education reform (Brunner \& Tillett, 2007). After the USSR collapsed, the official educational rhetoric of the Russian Ministry of Education emphasized decentralization, diversification, humanization, curricular relevance, learner-centered pedagogy, innovation, and the removal of ideological policy (Eklof \& Dneprov, 1993). However, unofficially, instructors within Russia simply paid lip service to this educational revolution, vocally expressing support for the decreed changes while maintaining existing practices in line with traditional Soviet educational policy (Eklof \& Dneprov, 1993; Elliott \& Tudge, 2007b).

The chaos of post-Soviet Tajikistan typified the challenges of providing quality education in Central Asia in the face of minimal support and resources-schools lost their heating, learners and instructors struggled with malnutrition, instructional time decreased overall, and some schools outright closed (Niyozov, 2006). In addition, the free-market economy privatized and streamed students into tracks that valued money and vocational skills over social and ethical sciences and general education (Niyozov, 2006). 
Ethical teaching often contradicted the realities of survival in a post-Soviet environment because ethical behaviors did not necessarily equate with material gains and success. Drugs and guns dominated the Tajik economy; the Soviet context left a legacy of corruption and nepotism; education was reduced to a pass-through, rendering honesty and hard work meaningless in an atmosphere of diminished opportunities (Niyozov, 2006).

In the twenty years following independence, nations throughout Central Asia have developed new educational models, developmental perspectives, and reform initiatives specific to their post-transition economies, government, and operational contexts (Nessipbayeva \& Dalayeva, 2013). The Russian Federation and the CAR have advocated for post-secondary education to help consolidate and modernize their societies (Brunner \& Tillett, 2007). The Russian Federation initiated its education reform with the 1992 Law of Education, calling for teachers to improve student opportunities for selfdetermination, democratic classrooms, and even "humanization" without offering any specific mechanisms or practices for doing so (Elliott \& Tudge, 2007a). The Russian education system increasingly emphasized competitiveness and individualism, codifying these values in a plethora of structural reforms that have created divisive educational hierarchies and inequalities (Federation, n.d.). Despite elaborate bureaucracies designed to improve academic quality, the Central Asian education systems all to some extent feature poorly trained teachers, limited instructional resources, weak management, and deteriorating facilities (DeYoung, 2006). Critics attribute this persistently declining academic quality to a lack of professional capacity and national investment in highquality education reform and a lack of consensus among stakeholders regarding the most effective means for raising quality (D. W. Chapman, Weidman, Cohen, \& Mercer, 2005). 
After the Soviet Union collapsed in 1991, Uzbekistan responded by upgrading the existing Soviet education system with structural reforms that decentralized and diversified education to align with international standards (Sia, 2014). While the Ministry of Higher and Secondary Specialized Education (MHSSE) develops educational policy and implements reform, Russia maintains significant influence within Uzbek education through its significant historical, social, and economic ties (Sia, 2014). The EU has also exerted considerable influence on Uzbek higher education reform through Tempus and Erasmus Mundus External Cooperation Programs, which stress the Bologna process that aligns with European education system standards, including Bachelor's, Master's, and Doctoral educational tracks (Sia, 2014).

The collapse of the USSR immediately raised several basic educational needs of critical importance, including establishing funding mechanisms for faculty and administrator salaries and locating replacements for Soviet textbooks and ideological curricula (Heyneman, 2010). To this day, transitional societies continue to struggle with professional capacity gaps; Central Asian ministries of education suffer from a scarcity of professional leaders, trained researchers, and policy experts who can evaluate, set, and design effective means for evincing positive educational outcomes (DeYoung, 2011). Despite the absence of Soviet control, educational leaders throughout Central Asia still turn to Russia in making key decisions, failing to recognize that Russia itself has yet to fully align educational institutions with the needs of a modern society and an international market economy (DeYoung, 2011). The post-Soviet status and work of teachers changed dramatically as they were no longer supported by a centralized government and command economy. The transition to independence left many teachers 
struggling to support themselves while remaining within the profession (Niyozov \& Shamatov, 2006). In the KR, teachers have supplemented their inadequate and unstable incomes through involvement in trading and commerce. Sometimes, the most experienced teachers elect to abandon the profession, sacrificing academic quality for financial stability and security (Niyozov \& Shamatov, 2006).

Throughout Central Asia, the Post-Soviet era has eroded the prestige, status, and respect of teaching as a profession (Silova, 2009). Consistent teacher shortages, the almost total feminization of the profession, an over-aged and underqualified teaching force, low professional service rates among teacher education program graduates, and fewer students entering such programs all impact the regional status of the profession (Silova, 2009). Students entering pre-service programs have comparatively low university examination scores compared to students in higher-demand programs, indicating that the lowest-performing students represent the future of teachers—and educational quality may continue declining as a result (Silova, 2009).

Institutions of higher education within the southern KR reflect the region's economic challenges and social conditions. Across multiple contexts, high-quality education at multiple levels has been correlated with economic development, social equality, and social stability (Byrd, 2012; Hanushek \& Woessmann, 2007; Sahlberg, 2006). Newly industrialized economies that have undergone rapid economic growth, including the Republic of Korea and Singapore, have shown evidence of adequate educational investment, sufficient human capital development, national commitment for education, and high expectations for educational attainment and quality (Byrd, 2012; Lee, 2007). 
The functional context of a post-Soviet higher education institution creates realities for students and instructors that challenge or even contradict Western conceptualizations of higher education. Most educators and students within the program and at the research site in the KR are completely unfamiliar with the concept of student engagement (Participant 01, personal communication, November 15, 2017). Declines in student learning, outcomes, and access/participation in the post-Soviet KR educational context substantiate the severity of educational issues in the nation (Mertaugh, 2004; Sainazarov \& Shamatov, 2010). The cumulative effects of rampant corruption, insufficient government funding, and the realities of life in this young, resource-deprived, and isolated nation further complicate attempts at improving education (Organization for Security and Co-operation in Europe, 2003; Ruget \& Usmanalieva, 2007). The KR needs an educated populace to help lift the entire population out of poverty, increase participation in the global economy, and enable the KR to maintain national sovereignty (U.S. Agency for International Development, 2014). Among the myriad consequences of the rapid decline in educational quality, the rise of radicalism and extremismparticularly among the burgeoning disenchanted and disenfranchised youth —-threatens to further destabilize the nation and region as well (International Crisis Group, 2016).

Russian students present somewhat of a mystery to researchers in that they indicate high levels of satisfaction despite higher education learning experiences that lack both rigor and quality (Chirikov, 2015). Minimal student engagement characterizes the modern Russian student experience even in top-tier universities. Traditional teaching methods foster passive learning; student-faculty interactions do not extend beyond lectures because faculty have challenging workloads and students display little interest; 
institutions tolerate or even accept academic cheating and corruption (Chirikov, 2015). Nonetheless, a recent analysis reported by Chirikov found that Russian students are generally satisfied with their higher education experiences (2015). Chirikov suggested that perhaps students are satisfied because they can obtain a degree without much challenge from the university, they perceive such academic quality as genuinely sufficient, or that the current reality of higher education meets their expectations (2015).

The modern Russian university can hardly be described as intellectuallystimulating or transformational, and predominant student attitudes and generalized passivity indicate that change is unlikely to arise from student empowerment. Previous comparative investigations within Russian student populations revealed positive student attitudes about learning, academic effort, and parent- and teacher-directed behaviors (Hufton, Elliott, \& Illushin, 2002). Student performance in specific subjects correlated with effort and teaching in those subjects, rather than an innate ability possessed by the students (Hufton et al., 2002). In the post-Soviet context, the independent Russian society has transformed student perceptions of motivation and engagement constructs, and these perceptions correlate with declining academic quality and student outcomes (Elliott \& Tudge, 2007a). In the post-Soviet higher education context, evidence indicates a half-truth to the commonly-held assumption that "postsecondary education completion with advanced competence hinges on active, consistent, and persistent engagement" (Lawson \& Lawson, 2013, p. 464).

\section{Education Reform in the Kyrgyz Republic}

Education is critical to developing social cohesion, the common bonds that unite a society (Aypay, 2004). As the modern KR continues to transition from its Soviet past, 
educational practices and policies oriented around democracy, free market economics, and globalization have the potential to socialize learners about how to function within and contribute to their developing nation (Aypay, 2004). Education reform requires support at every level: individuals who are committed to providing quality leadership, an eagerness for self-development, creativity in managing and educating, voluntary financial transparency, and an achievement orientation (McLean, Karimov, \& Asankanov, 2004). Education reform stumbles with any form of ineffective leadership: cronyism, unqualified leaders with limited practical experience, or a general lack of accountability, incentives, and resources (McLean et al., 2004).

The increasingly visible threats of national destabilization and international terrorism have motivated the Kyrgyz government to mandate education reforms and support the efforts of international aid organizations (U. Nazarov, 2016). Previous attempts at education reform, from primary through post-secondary education, have been disjointed and produced only modest, small-scale improvements (Heyneman \& DeYoung, 2004). In repeated administrations of the PISA examination, students from the KR ranked last among all participating countries in basic academic skills, demonstrating the severity of the country's education deficits (Sainazarov \& Shamatov, 2010). By using alternative evaluations to assess student learning, instructional standards, and graduate outcomes, we can theoretically identify strategies for improving institutional policies and instructional praxis, ultimately improving education quality for future generations.

While access to education in the KR has been maintained — and, in the case of higher education, improved to near universal access—-student attendance, learning, and 
outcome measures indicate that quality continues to decline, even for basic education content and skills (Organization for Security and Co-operation in Europe, 2003; Smolentseva, 2012). The 2013-2017 National Sustainable Development Strategy (NSDS) for the KR called for further systemic education reform, lauding academic quality as one of the most reliable indicators for the future development of any nation (National Council for Sustainable Development of the Kyrgyz Republic, 2012b). In 2002, the Concept of Education, an official decree of the Kyrgyz government, advocated specifically for improved educational equality, accessibility, and quality; it assigned responsibility for this to the Ministry of Education and Science (Nessipbayeva \& Dalayeva, 2013). The most recent NSDS also called emphatically for rapid implementation of the Education Development Strategy (EDS) for 2012-2020, and excoriated the current state of education for consistently lagging behind basic human development needs and hindering improvements (National Council for Sustainable Development of the Kyrgyz Republic, 2012b). The NSDS and the EDS called for improving quality assurance, revising funding mechanisms, allowing market forces to govern post-secondary systems, improving research and technology, and raising instructional standards and professional quality, among other reforms (National Council for Sustainable Development of the Kyrgyz Republic, 2012a; National Council for Sustainable Development of the Kyrgyz Republic, 2012b). The NSDS specifically highlighted corruption as the most acute problem within higher education, stating that it alone can erode and destroy the national foundation by hindering change and advancement (National Council for Sustainable Development of the Kyrgyz Republic, 2012b). Even in modern-day higher education, the KR has an illustrious record of 
extensive corruption that invalidates academic meritocracy, detracts from the earning abilities of educated individuals, and reflects the degree of corruption at the national level (Osipian, 2009). The effects and hindrances posed by corruption must be accounted for in any discussion of reform efforts, national priorities, and desired outcomes.

National education reform initiatives within the KR have consistently prioritized quality improvement without specifying the means to do so, much less providing the required funding or resources. In one instance the stavka teaching load compensation scheme for instructors, despite encouraging evidence for quality improvement, was adopted and fully abandoned in less than two years' time (Gita, 2016; UNICEF CIEES, 2011). The government devotes little attention to consistency in education reform; it has broadened the definition of "education quality" to include teacher quality, attendance, and materials while switching to indirect measures of actual student achievementthereby making it easier to claim improved quality (D. W. Chapman et al., 2005). The PISA and other reliable measures of student achievement have been set aside, largely because the results were poor and publicized continuing declines which negatively represented the national education system and the nation itself (Sainazarov \& Shamatov, 2010). Meanwhile, quality improvement standards are repeatedly adopted but not implemented, textbooks are developed but remain undistributed and prohibitively expensive, and teachers suffer from decreased salaries, proficiency, and training (D. W. Chapman et al., 2005).

Basic operational challenges - such as maintaining functional learning facilitiesoften trump initiatives to improve student learning experiences, slowing down reform efforts as a whole (Mertaugh, 2004). In addition, student variability compounds 
instructional challenges; students enter higher education with a wide range of learning skills, academic abilities, and even native languages. To improve the fundamental quality of post-secondary education within the KR, reform initiatives must be tailored to the region's distinct educational realities and pervasive socioeconomic challenges (DeYoung, 2005).

USAID has sponsored numerous education reform projects and initiatives throughout the Central Asia Republics and Post-Soviet sphere ("Country Development Cooperation Strategy,” 2017). A recent Quality Learning Project (QLP) within the KR sought to improve teacher training in student-centered methodologies and formative student assessments, promote teacher involvement in curriculum reform, and increase the effectiveness of the education finance system through teacher salary reform, among other initiatives (Ginsburg, 2010). Consistent with the existing literature and previous evaluations, this program assessment reported that student-centered methodologies had not achieved many successes within pre-service teacher training institutions and pedagogical universities (de la Sablonnière et al., 2009; Tate et al., 2011). While the QLP holds clout in specific communities and institutions, additional stakeholders must use their local ownership and leadership in education reform to evince sustainable social transformation (Semere, 2015).

Critically, donor agencies such as the UN, USAID, and the World Bank have focused exclusively on the Education for All platform, emphasizing basic education over secondary, tertiary, or vocational education in developing countries (Heyneman, 2009). While basic education is important, the narrow focus on this one area has undermined the rationale of more advanced education and training, diminishing the professional respect 
and economic rationale traditionally associated with these agencies (Heyneman, 2009). They have relegated themselves to mere charitable handouts for rural pupils, rather than as facilitators for the construction of the professional capacities and infrastructure necessary for open and democratic societies (Heyneman, 2009).

The Kyrgyz government's NSDS focuses on attenuating the decline of basic educational quality (National Council for Sustainable Development of the Kyrgyz Republic, 2012a), and even the modest goals presented in the plan are challenging for a nation that has neglected and defunded its education for twenty years (ADB, 2015). Limited instructional materials, dilapidated facilities, and underpaid/untrained instructors among a rapidly expanding and young population create significant areas for improvement, but with very few resources to do so (Education, Audiovisual and Culture Executive Agency, 2013). With regard to post-secondary education, the strategic plan highlighted inadequate skills and knowledge among graduates, low credentials and qualifications of faculty, and an inefficient system of quality assurance, among other major problems (National Council for Sustainable Development of the Kyrgyz Republic, 2012a). The government does not routinely deploy the existing national education quality assurance mechanisms to certify public or private higher education institutions; licenses are not contingent on instructional quality or institutional improvement because "there are currently no criteria or standards for evaluation"- a clear indication of the severity of challenges to education reform (National Council for Sustainable Development of the Kyrgyz Republic, 2012a, p. 25).

A stable, improved education system should promote economic growth, social equality, and social stability (Hanushek \& Woessmann, 2007; Levy \& Hawkins, 2009). 
Of course, this proposition rests on an underlying assumption that student engagement and its resulting outcomes are essential for personal development and social advancement. This assumption may prove to be an optimistic bias inherent to the Western higher education and the construct of student engagement, which does not reflect the realities of the KR (Pfeffer, 2015). In addition, improved education could theoretically introduce unintended consequences, even destabilizing the fragile balance of social and economic conditions under which this young nation currently operates.

\section{College Student Success}

Tinto (2006) focused on aspects of student success, including retention, institutional action, and program implementation, to identify significant barriers to equitable and inclusive higher education. He also argued that student retention and thus success are educational matters that revolve around the classroom — the one place where institutional action can directly impact learning, retention, and graduation (Tinto, 2010). Critically, Tinto identified the essential components for retaining students: expectations, support, assessment and feedback, and involvement $(2010,2012)$. Of course, these higher education institutions function in changing conditions that make it formidably challenging to operationalize these components into discrete institutional actions and educational practices (Tinto, 2010). Institutional action must center on the classroom to transform access to higher education into positive student outcomes, such as higher rates of college completion and student success (Tinto, 2012). A review of the existing student success literature reveals that individual choice and motivation play key roles in success; Thelin (2007) argues that life choices, including those surrounding post-secondary education, are often probabilities shaped by collective forces beyond one's exclusive 
control, and Tinto (2012) posits that an institution of higher education can (and should) do only so much to promote student success if individuals themselves elect not to invest and participate.

The overlapping yet distinct concepts of student engagement, student involvement, and student integration together define various aspects of student success, and these differing aspects of student success have continued to evolve with ongoing research and development (Wolf-Wendel, Ward, \& Kinzie, 2009). "Engagement" encourages institutional reflection on practices and activities that contribute to student learning, and is grounded in empirical indicators of good practice in undergraduate education, involvement theory, and quality of effort measures (Wolf-Wendel et al., 2009). "Involvement" focuses on the individual and his or her activities, including both academic and extracurricular activities, and has been linked to almost every positive outcome (Wolf-Wendel et al., 2009). "Integration" revolves around how students perceive the outcomes of a campus's alignment with (or against) a particular culture; it considers student relationships and reciprocal commitment between the student and the institution (Wolf-Wendel et al., 2009). These three concepts overlap some in theoretical content but should not be used interchangeably, as Pascarella and Terenzini (2005) and other researchers have, as this further confounds the concepts. Wolf-Wendel, Ward, and Kinzie (2009) interviewed several major researchers in the field and reflected on the implications for both theory and practice, noting that each concept contributes something unique to student development, and that the development of common definitions and clear terminology is essential for furthering research into student engagement. 
Pike (2013) traced the origins of student engagement to the work of Ralph Tyler, Robert Pace, Alexander Astin, Chickering and Gamson, and George Kuh. Tyler coined the term "evaluation" within educational research and referenced engagement in several of his most famous works (Tyler, 1932). He argued that educational experiences must be responsive to three factors: 1) the learner, including their developmental factors, interests and needs, and life experiences; 2) society, including values and aims, attitudes, and guiding principles; and 3) subject matter knowledge, particularly what is deemed acceptable and practical (Tyler, 1932, 2013). Pace claimed that quality of student effort was the single greatest determinant of positive student outcomes (Pace, 1982). He argued that while prospective students choose higher education institutions based on facilities, personal and social opportunities, and undergraduate experience, their experiences as actual students stemmed most from their activities, not from the institution itself (Pace, 1982, 1984). Astin emphasized that development and success follow directly from student involvement, specifically the quantity and quality of physical and psychological energy that students devote to academic experiences (Astin, 1984). Astin's theory also placed the burden of student involvement, development, and success on the institution's policies and behaviors of its faculty and staff $(1984,1993)$. Chickering and Gamson's often-cited seven principles of effective undergraduate education operationally define student engagement by highlighting student-staff contact, active learning, prompt feedback, time on task, high expectations, respect for diverse learning styles, and cooperation among students (1987). Finally, Kuh, Schuh, and Whitt observed institutional aspects of student life and learning that fostered the development of a campus culture conducive to active student participation and positive student outcomes 
(1991). Three main factors were identified that supported an active campus life: 1) a clear, coherent philosophy that sets expectations for student behavior and guides campus policy and practice development; 2) a campus culture that encourages student participation and loyalty; and 3) faculty, staff, and administrators who commit to the importance of out-of-class experiences in achieving institutional aims (Kuh, 1991; Kuh, Schuh, \& Whitt, 1991). The conceptual complexity of student success incorporates multiple dimensions, factors, and variables in approaching analysis of the construct. This complexity is in turn reflected within the spectrum of definitions and perspectives comprising the ascribed components of student success in higher education and the chief focus of this study, student engagement.

\section{Student Engagement}

A sizable body of literature, research, and practice of higher education has assumed that student engagement, in various iterations, is essential for student learning, development, and ultimately success. Shulman (2002) goes so far as to postulate a taxonomy of learning in education that "begins with engagement, which in turn leads to knowledge and understanding” (p. 38). Although the student engagement construct is an increasingly common research focus, the existing literature continues to debate its conceptual and operational definitions. The diversity of definitions reflects the depth of higher education research concerning student engagement but makes the construct susceptible to misinterpretation as a panacea for educational efficacy and reform — many within the field have criticized various applications and interpretations of the construct on multiple grounds. A unified definition of student engagement, whether conceptual, theoretical, or operational, has yet to be developed. 
Definition. Coates (2007) defined student engagement as a "broad construct encompassing salient academic and nonacademic aspects of the student experience" (p.122). Krause and Coates (2008) elaborated on this definition, emphasizing its primarily educational role and the underlying assumption that researchers can identify beneficial activities and conditions associated with learning. Mandernach (2015) differentiated between student engagement as a process and as a product; the process analyzes learning relative to behaviors, activities, and attitudes, while the product focuses the learners' resulting cognitive and affective states. Some argue that student engagement is not a unitary construct, but rather an umbrella term for a family of ideas that research has linked empirically with desired outcomes in higher education (McCormick et al., 2013). Others have conceptualized student engagement as a metaconstruct that integrates multiple lines of inquiry and intellectual traditions (Fredricks, Blumenfeld, \& Paris, 2004). One recent study defined student engagement relative to academic motivation: motivation encompasses the personal inclination, energy, emotion, and drive needed to learn, work effectively, and achieve, while engagement is the behavior that reflects motivation (Martin, Ginns, \& Papworth, 2017). This same study indicated that motivation and engagement have a cyclical relationship, with one perpetuating the other (Martin et al., 2017). Additionally, there are many opportunities to optimize motivation and engagement, every moment of every day for every student with activity- and person-centered approaches promoting positive student outcomes through quality pedagogy (Martin et al., 2015).

Several interpretations of student engagement within the higher education context approach it as an evolving, multidimensional construct composed of institutional 
practices and student behaviors that lead to positive educational outcomes (Kahu, 2013).

Kuh (2009) defined student engagement as "the time and effort students devote to activities that are empirically linked to desired outcomes of college and what institutions do to induce students to participate in these activities" (2009b, p. 683). More generally, $\mathrm{Hu}$ and Kuh (2002) interpreted student engagement as a function of the interaction of students with their institutions. This definition of the construct has evolved over time to more adequately represent the complexity of relationships between outcomes, investments of time and effort, and educationally-purposeful activities (Kuh, 2009). Leach and Zepke (2011) identified four primary perspectives in the literature that contained several key tenets of student engagement: motivation and agency, studentteacher transactions, student-student interactions, institutional support, active citizenship, and non-institutional support. Kahu (2013) proposed an inclusive sociocultural perspective stating:

The socio-cultural perspective offers important ideas on 'why' students become engaged or alienated at university, with a particular emphasis on non-traditional students. It highlights the need for the institutions to consider not just the student support structures but also the institution's culture, and the wider political and social debates impacting on student engagement. It adds, therefore, a critical and often neglected piece to the task of understanding student engagement (p. 764).

By viewing student engagement through different ideological perspectives, it is possible to gain a better understanding of the relationship between the construct and important educational outcomes, including student development, learning, and retention (Hagel, Carr, \& Devlin, 2012). Higher education policy and practice stands to gain from a broader understanding of student engagement as a process with multiple dimensions, and quantitative and qualitative methods can assess whether measures, scales, and factors hold true in international contexts (Krause \& Coates, 2008). Consistency and clarity 
within the conceptualization and measurement of student engagement are imperative for both research and practice (Appleton, Christenson, \& Furlong, 2008). The construct is multidimensional and sensitive to context, so the literature advocates for a more nuanced and holistic conceptualization of student engagement that is both efficacious and relevant to educational reform (Fredricks et al., 2004).

Dimensions of student engagement. Cognition, behavior, and affect/emotion consistently surface in the literature as key dimensions of student engagement. Elaine Chapman (2003) summarized the contributions of each dimension with cognitive indices for mental effort and initiative in coursework, behavioral indices for individual participation and group actions, and affective indices for personal investment and emotional reactions. She rather eloquently stated that the construct includes students' cognitive investment in, active participation in, and emotional commitment to their learning (E. Chapman, 2003). Butler (2011) aligned typical assessment indicators along the behavioral, cognitive, and affective domains and stressed that the subject of analysis must also be clarified prior to assessment.

Behavioral. Fredricks, Blumenfeld, and Paris (2004) identified three common definitions for the behavioral dimension of student engagement: 1) positive conduct, including compliance with rules, adherence to norms, and avoidance of disruptive and negative behaviors; 2) involvement in learning and academic tasks behaviors such as effort, persistence, concentration, attention, question asking, and contributing to class; and 3) participating in school-related activities, such as athletics or governance. Fredricks and Eccles noted that academic outcomes and student development improved with an increase in activity breadth, diversity in activity contexts, and the timing of 
involvement (2006a, 2006b). Since larger universities typically restrict the amount of student-faculty interaction, some studies have indicated that student preparedness and interest in the curriculum may be more effective indicators of behavioral engagement (Lutz \& Culver, 2010).

Cognitive. Fredricks et al. postulated that the cognitive dimension of student engagement draws from two areas: psychological investment in learning/motivation and cognition/strategic learning (2004). Here, a student's investment is his or her thoughtfulness and willingness to try to develop difficult skills and comprehend complex topics (Fredricks et al., 2004). Cognitive engagement incorporates individual characteristics including motivation, self-efficacy, and expectations (Jimerson, Campos, \& Greif, 2003). Citing a fundamental definition advanced by Newmann, Wehlage, and Lamborn (1992), Kahu (2013) referred to cognitive engagement as “a student's psychological investment in and effort directed towards learning, understanding, or mastering the knowledge, skills or crafts" (p. 12).

Emotional. Affect in student engagement includes multiple components such as interest, boredom, happiness, sadness, anxiety, belonging, interest, and value (Fredricks et al., 2004). Thus, students define emotional engagement through their positive and negative reactions to instructors, peers, and the academic context (Fredricks et al., 2004). One study found emotional intelligence to positively predict both cognitive and emotional engagement among students (Maguire, Egan, Hyland, \& Maguire, 2017). Johnson et al. conceptualized this dimension as attachment, including the behaviors, attitudes, and feelings of belonging relative to an educational institution (Johnson, Crosnoe, \& Elder Jr, 2001). 
Critiques of student engagement. Kuh $(2009$, p. 15) contended that the student engagement construct has been misinterpreted and misused, calling on practitioners and researchers to clarify which "forms of engagement work best under what circumstances for different groups of students" (p. 15). In addition, although it is a complex and multidimensional construct, most predominant definitions focus on the behavioral perspective, do not factor in sociocultural influences, fail to distinguish between student engagement as a process and as a product, and ignore its antecedents and consequences (Kahu, 2013). Zepke noted the lack of critical research on the concept and inquired whether, in trying to be everything in both teaching and learning, the construct ends up ignoring context and fitting only the most generic learners (2014). He also suggested that the most engaging classrooms may espouse pedagogy at the expense of curriculum, positing that the popularity of student engagement stems from its neoliberal ideology, instrumental view of knowledge, and the emphasis on performance and accountability within modern higher education (Zepke, 2014).

Student engagement assessment. Student engagement data collection methods take many forms including student self-report, experience sampling, teacher ratings of students, interviews, direct observations, checklists and rating scales, work sample analysis, and focused case studies (Mandernach, 2015). Instruments for assessing student engagement are available at the institutional and course (program) levels; institutional data quantifies student engagement in the cumulative learning process, while program level data assesses the impacts of learner-centered pedagogical methods on student success (Butler, 2011). There are four subdivisions of course level student engagement assessments: 1) informal, formative assessments, including instructor observations; 2) 
administrative data; 3) student self-reports; and 4) formal, summative assessments including standardized survey instruments and scales (Mandernach, Donnelli-Sallee, \& Dailey-Hebert, 2011).

Applications of student engagement. Student engagement as an educational concept and practical guide has numerous applications within higher education praxis. Kuh (2009) bluntly stated that "institutions cannot change who students are when they start college" (p. 14); therefore, assessments are valuable because they help evaluate and improve teaching and learning so that the institution can then assist students in their pursuits of educational and personal goals. Similarly, Coates asserted that it is most valuable to assess student learning activities, which can then be used to evaluate and manage the quality, nature, levels, and targeting of resources (2007). Krause and Coates (2008) elaborated that student engagement provides sufficient means for "guiding higher education research policy and practice" (p. 493) and "determining whether students are engaging with their study and university learning community in ways likely to promote high-quality learning" (p. 493). Mandernach (2015) argued that most measures of student engagement include elements of both process and product by examining how learners' roles in the learning process affect cognitive and affective positions. Within the framework of higher education quality assurance and improvement, Chang (2012) posited that the multidimensional nature of the student engagement construct offers a way to improve undergraduate learning and educational experiences.

The application of student engagement as a focus for academic quality enhancement and educational reform is well established and thoroughly researched (McCormick et al., 2013). In one study, first-year institutional efforts to facilitate and 
enhance student engagement with educationally effective practices aided students with the transition into higher education by empowering them to learn actively (Everett-Heath, 2003). There is debate in the literature about the most effective means for fostering student engagement, ranging from conceptual to theoretical to operational actions that vary in efficacy and practicality (Zepke \& Leach, 2010). Altering student perceptions of the campus environment seems to encourage student engagement at the institutional level (Hu \& Kuh, 2002). Bowen (2005) outlined four applications of student engagement and described the learning types associated with each: learning process (active), object of study (experiential), context of study (multidisciplinary), and human condition (service).

Trowler and Trowler (2010b) detailed six positive outcomes associated with the construct noting: 1) improved learning; 2) increased retention and graduation rates; 3 ) advanced equality and social justice; 4) improved curricular relevance; 5) strengthened educational institutions, and 6) bolstered recruitment and institutional marketing. The foundational level of importance attributed to engagement was reiterated by Shulman (2002) relative to learners in stating:

Learning begins with student engagement, which in turn leads to knowledge and understanding. Once someone understands, he or she becomes capable of performance or action. Critical reflection on one's practice and understanding leads to higher-order thinking in the form of a capacity to exercise judgment in the face of uncertainty and to create designs in the presence of constraints and unpredictability. Ultimately, the exercise of judgment makes possible the development of commitment. In commitment, we become capable of professing our understandings and our values, our faith and our love, our skepticism and our doubts, internalizing those attributes and making them integral to our identities. These commitments, in turn, make new engagements possible -- and even necessary (p.38).

\section{Kahu's Sociocultural Approach to Student Engagement}


As detailed previously, there are many conceptualizations of the student engagement construct encompassing multiple perspectives and dimensions. For this study, the conceptual framework advanced by Kahu (2013) moves beyond the institution and approaches student engagement as "a psychosocial process, influenced by institutional and personal factors, and embedded within a wider social context” (p.768). Fredericks et al. (2004) presume student engagement to be "malleable, responsive to contextual features, and amenable to environmental change" (p. 59); this interpretation lends additional support to the inclusion of contextualist theory. In addition, the framework distinguishes between antecedents, student engagement, and consequences, introducing a logic model incorporating multiple variables and impacts upon student experiences (Kahu, 2013). This broader conceptualization of student engagement accommodates the sociocultural and socioeconomic factors challenging the KR and its higher education system as it adapts to a market economy in an increasingly globalized world (ADB, 2015; DeYoung, 2010).

The conceptual framework developed by Ella Kahu (2013) views the student engagement construct as a function of six elements which are identified and discussed in the context of this study as follows. First, Kahu stressed the impact of the greater sociocultural context on the construct, in this case the political and social environment of the KR including the impact of Kyrgyz culture, official higher education policies, and the state of the local economy (2013). Second, Kahu (2013) included structural influences, which here consisted of the university itself, its organizational culture and policies, the program itself, as well as students' backgrounds, supports, and families. Third, Kahu approached psychosocial influences, which in this instance included post-Soviet higher 
education practitioners, their support and workloads, student relationships, and their individual motivation, skills, identity, and self-efficacy (2013). Fourth, she centered the framework around state of student engagement, along with its behavioral, cognitive, and emotional domains (Kahu, 2013). Fifth, Kahu identified proximal consequences, these consisted of program educational quality and achievement as well as satisfaction and well-being among stakeholders (2013). Finally, Kahu (2013) included distal consequences, the long term academic and social outcomes associated with promoting student engagement. Aside from student success, improved program outcomes, and social improvements, Kahu (2013) also emphasized benefits to citizenship and social stability, both of which were identified as key areas for improvement in the KR.

\section{The National Survey of Student Engagement (NSSE)}

NSSE permeates higher education research, policy, and practice and has emerged as a definition in and of itself for the student engagement construct (Bowen, 2005). The main instrument within the NSSE is The College Student Report (CSR), a self-reported instrument that indirectly measures learning outcomes via student engagement proxies or process indicators: student behaviors, institutional actions and requirements, reactions to college, and student background information (Kuh, 2005; Kuh, 2009). The CSR contains benchmarks of effective educational practice that comprise an operational definition of student engagement as measured by levels of academic challenge, active and collaborative learning, student-faculty interaction, enriching educational experiences, and supportive campus environment (Kuh, 2003). This operational definition of student engagement is complicated by additional dimensions — with statistical validity equivalent to the original five benchmarks - that surfaced in data obtained through the CSR 
(LaNasa, Cabrera, \& Trangsrud, 2009). In other words, the NSSE benchmarks do not necessarily have the statistical strength to claim predictive validity for student outcomes (Hagel et al., 2012).

Critically, NSSE does not directly measure student learning or the psychological state of engagement (Wefald \& Downey, 2009). The NSSE instrument collects selfreported information and aligns this data with benchmarked effective educational practices to approximate academic quality (Kuh, 2009). Although empirical evidence lends support to this conceptualization of engagement, it does not change the reality that the whole instrument is a gross approximation: the NSSE uses one indirectly-assessed proxy (student engagement) as the proxy for yet another entity (academic quality). While the NSSE and the CSR have been administered within international contexts, most associated institutions have been Western-oriented, relatively well-funded, and Englishspeaking in lingua franca or at least in official instruction.

The underlying conceptual framework of the NSSE rests on several assumptions: that what students actually do during college matters more than who they are or where they attend, that the time and energy dedicated to educationally-purposeful activities is the single best predictor of learning and personal development, and that institutions that engage their students more fully in educationally purposeful activities can claim to be of higher quality (Kuh, 2005). As noted previously, Kuh (2009, p. 14) advocates for additional research investigating "what forms of engagement work best under what circumstances for different groups of students" $(2009$, p. 14). This statement presupposes that specific forms of student engagement will improve outcomes and education quality for certain students under certain circumstances. Such a critical assumption could 
contradict or negate the intents of higher education reform. Additionally, any assessment of educational quality, particularly at the post-secondary level, cannot be separated from students' varying expectations and goals, thus biasing the NSSE toward traditional Western college students (Lerer \& Talley, 2010).

Peter Ewell developed the NSSE and associated benchmarks with a design team that originally included Alexander Astin, Gary Barnes, Arthur Chickering, John Gardner, George Kuh, Richard Light, and Ted Marchese (Kuh, 2009). They derived many of the NSSE's items from existing student surveys, including the College Student Experiences Questionnaire (CSEQ) with which Kuh was affiliated (Gonyea, Kish, Kuh, Muthiah, \& Thomas, 2003), the Cooperative Institutional Research Program (CIRP) Freshman Survey developed by Astin (“CIRP Freshman Survey - HERI," n.d.), and surveys from the University of North Carolina System ("NSSE Origins and Potential," n.d.). Content validity, or the degree of alignment and performance between test questions and subject area, is often decided by experts in the relevant content areas ("Validity Evidence Research - The College Board," n.d.). It is concerning that a homogenous, traditional, academic group of researchers developed an instrument that purportedly applies to the diversity of college student experiences. For all its accolades as evidence-based practice, the NSSE benchmarks possess surprisingly little direct evidence for content validity beyond the value judgements of experts from a traditional, and arguably outdated, university context.

NSSE benchmarks of effective educational practice. The CSR assesses student engagement based on two premises: that learning and success are related to the amount of time and effort students devote to educationally-purposeful activities, and that institutions 
can use their resources to influence the degree of student engagement (Kuh, 2003, Kuh, 2006; Pike, 2013). The NSSE defines effective educational practice with five institutional benchmarks, or clusters of desirable student behaviors and institutional actions (Kuh, 2000; Pike, 2013). According to Kuh (2000) and Pike (2013), the NSSE benchmarks are designed to summarize an institution's educational practices and facilitate improvements in the quality of undergraduate education. The five benchmarks are detailed below.

Level of academic challenge. This benchmark links student learning with collegiate quality, defined by the degree of challenge in intellectual and creative work, amount of assigned academic work, complexity of tasks, and faculty standards in student evaluations (Kuh, 2000). The CSR evaluates this benchmark with questions about how students prepare for class, quantifying the amount of reading and writing, use of higherorder thinking skills, and the effort students put toward meeting faculty standards (Kuh, 2000).

Active and collaborative learning. This benchmark posits that students learn best with intense involvement and opportunities to apply their studies in multiple contexts (Kuh, 2000). Student collaboration fosters practical problem-solving skills (Kuh, 2000). The CSR inquires how often students ask questions, contribute to class discussions, make presentations, prepare assignments with classmates outside of class, collaborate on projects in class, mentor, tutor, teach other students, participate in community-based projects, and discuss course content with others (Kuh, 2000).

Student-faculty interaction. By interacting with faculty on research projects, on committees, and in organizations, students witness how experts identify and solve 
problems (Kuh, 2000). In general, these interactions correlate with positive outcomes by enabling faculty to become role models, mentors, and guides for personal and professional development (Kuh, 2000). The CSR evaluates the extent to which students meet with faculty outside of class to discuss grades or assignments, career plans, or ideas from their courses; to work on activities, including research, beyond the scope of the coursework; and to receive prompt feedback on academic performance (Kuh, 2000).

Enriching educational experiences. Effective institutions create a variety of learning opportunities to complement academic program goals, supporting components of student diversity, technology, and project-based learning (Kuh, 2000). According to the NSSE, these experiences result in learning that is deeper, meaningful, and useful because students integrate content into their own identities (Kuh, 2000). The CSR evaluates whether the institutional climate actively fosters interaction, and inquiries about the frequency of student discussions with others who have different beliefs, opinions, and values; interactions with different races and ethnicities; applications of technology in assignments; and participation in project-based learning (Kuh, 2000).

Supportive campus environment. Student performance and satisfaction improves when institutions commit to promoting student success and nurture positive relationships between student groups (Kuh, 2000). The CSR assesses the campus environment by asking whether students feel supported to succeed academically and thrive socially, cope with non-academic responsibilities, and form positive relationships with their peers, faculty, and administration (Kuh, 2000).

Critiques of NSSE benchmarks. The NSSE benchmarks favor the experiences of traditional students, so institutions that diverge from this norm—especially those with 
large numbers of non-traditional students—receive negatively skewed marks for academic quality solely on the basis of their student population (Lerer \& Talley, 2010). Because the NSSE draws on correlational analyses with potential confounding influences, researchers cautiously claim that NSSE results are merely proxies for growth in institutional outcomes (Pascarella, Seifert, \& Blaich, 2010). The validity of selfreported instruments such as the CSR is questionable as well. Porter highlighted four key weaknesses in the NSSE: 1) Empirical, rather than theoretical, concerns drive the broad conceptual domain of the NSSE; 2) College students must estimate their behaviors, creating the potential for errors; 3) The dimensional structure of the NSSE has not been replicated by other researchers and lacks reliability; and 4) Instrument scales are largely uncorrelated with objective external measures of student learning (Porter, 2011; Porter, Rumann, \& Pontius, 2011).

\section{Evidence-Based Educational Practice}

Lying at the intersection of research, policy, and practice, education presents a challenge for evidence-based practice because it is difficult to evaluate effectiveness without also making a value judgement (Biesta, 2007). Several features of evidencebased practice restrict decision-making including: positivist epistemology, a correlation with the practice of medicine, the role of research in professional action, and the implied practical role of research (Biesta, 2007). From this perspective, evidence-based decisionmaking relies on researchers rather than policy makers or practitioners to define efficacy (Biesta, 2007). Education can be viewed as a teleological practice guided by an aim or purpose; actions and effective practices must be applied with recognition of their desired outcomes (Biesta, 2010). The literature reflects this observation. Experts support high- 
impact practices to promote student engagement simply because such practices correlate with positive outcomes for students, even though we lack knowledge of the outcomes, their assessment, and their associated intents (Brownell \& Swaner, 2009). In addition, international donor agencies often praise learner-centered pedagogy, with claims of educational and cognitive benefits but significantly it can also be interpreted as subtly promoting democratic ideology (Tabulawa, 2003).

\section{Schön's Reflective Practice Model}

Donald Schön advanced professional reflection for developing expertise in addressing problems that presented contextual and practical challenges to the application of traditional research-based evidence in practice (1983). Schön's epistemology of reflective practice posited that techno-rational, positivist learning was not adequate for addressing complex organizational and social problems (1983). Schön (1983) called for the development of reflective practitioners to analyze problems within learning organizations to continuously develop solutions to problems as changes occur and circumstances evolve. In referencing Schön, Trowler and Trowler (2010a) advocated that evidence-based practice must rest on sound evidential and theoretical bases, substantiated by ground level cognitive work by reflective practitioners, before it can be leveraged to create change. Schön advanced reflective analysis as a tool for developing artistry and expertise in practice and responding in challenging contexts where evidence derived from research and theory has proven ineffective (Schön, 1983).

In contexts defined by complexity, instability, and uncertainty, such as the research site under analysis, traditional evidence-based practice faces considerable challenges to effectiveness (Schön, 1983). In the problem setting process, one defines the 
decision at hand, the desired ends, and the means to do so (Schön, 1983). Reflecting-inaction analyzes current praxis in context. Knowing-in-action identifies attempts to apply evidence from other contexts or situations. Reflecting-on-action uses participant experiences to gauge how practice can and should be changed (Schön, 1983). Individuals develop artistry in expertise as reflective analysis helps them review their own practices, theories, and knowledge relative to their professional roles.

Higher education professionals must make a considerable time commitment to apply reflective practice within praxis. Even the most genuine reflective efforts are hindered by deeper-seated problems such as ongoing budget cuts; expanding commitments and responsibilities; conflicting institutional, professional, and personal priorities; and a general lack of motivation (Davis, 2003). Also, mastery of the reflective process - specifically, using reflecting-in-action and reflecting-on-action to develop professional problem-solving expertise — does not necessarily correlate with experience (Ferry \& Ross-Gordon, 1998). Educators face additional challenges in the vaguelydefined terminology of epistemic cognition, the complex external and institutional forces that shape instructional requirements, and the reflexive relationship between context, specific tasks, and the significance of the problem (Alexander, 2017).

Soviet-era documents repeatedly stated that higher education should adopt a utilitarian and instrumental focus on training advanced specialists as part of the greater Soviet Plan (Smolentseva, 2017). Soviet decrees called for improved and targeted use of specialists, indicating probable misalignment between graduate qualifications and the workplace (Smolentseva, 2017). The industrial setting thrived on the combination of theoretical and practical training, motivating collaboration between higher education and 
the production sector; this alliance yielded theoretical developments with practical applications for the benefit of industry, but not economic or social development (Smolentseva, 2017).

The NSSE benchmarks of active and collaborative learning and student-faculty interactions have direct applications within reflective analysis (Kuh, 2000). Schön's concepts of problem setting, theory-in-practice, and knowledge-in practice encompass the ways that students and faculty interact to identify and solve practical problems. The description for active and collaborative learning explains that student interactions beget skills essential for messy, unscripted problems in daily life. In his observation that techno-rationality fails to accommodate loosely defined problems within unstable contexts, Schön specifically advocates for the development of such practical problemsolving skills. The NSSE benchmarks incorporate many aspects of reflective analysis as opportunities for students to synthesize, integrate, and apply knowledge obtained via deep and meaningful learning.

To conduct the reflective cognitive work necessary to bridge the gap between student engagement research and higher education practice, a framework for understanding the assumptions, goals, and values present throughout this post-Soviet context was needed. In an environment such as the KR where economic survival remains a concern and government services and social supports are lacking, emphasis is often placed on resolving surface problems without addressing their underlying causes (Ruget \& Usmanalieva, 2007). Relative to KR higher education reform, it has been demonstrated time and again, that when well-intentioned interventions interrupt too many power structures too quickly or severely, or best practices jeopardize livelihoods obtained 
through corruption, those reforms and their supporters are silenced and the status quo prevails (DeYoung, 2004). By exploring practice within a post-Soviet higher education institution among practitioners and students, the underlying causes behind many problems can be revealed.

\section{Summary}

This chapter presented the foundation of literature that guided me through the development of this dissertation. The research site operates in a fragile post-Soviet reality, and it is important to understand the people and politics of pre-Soviet Central Asia, life within Soviet Kyrgyzstan, and the realities of the present-day post-Soviet KR. Within each of these eras, I focused the discussion on attempts to provide quality education. The KR has participated in and bore witness to varied efforts at education reform that foreshadowed the persistent challenges in developing effective practices via quality improvement initiatives. I then reviewed the literature on student success and student engagement, including the construct's development, applications, and critiques. I highlighted the narrow, Western interpretation of higher education from which experts developed the NSSE and empirically identified and substantiated the five benchmarks of effective educational practice; this gave way to discussion about critiques of evidencebased practice. Finally, I explored how components of reflective analysis fit into this examination of student engagement and steer the analysis. In Chapter Three, I detail the ethnographic case study design and qualitative research methods selected to collect and analyze data to answer the research question and address the gap in the existing literature. 


\section{CHAPTER III}

\section{METHODS}

This study investigated the experiences and reflections of higher education practitioners in a teacher-preparation program in the Kyrgyz Republic (KR) about Western higher education practices associated with student engagement. In this research design and methodology chapter, I detail the ethnographic case study conducted and qualitative field research methods employed while reiterating the contextual constraints, extant literature, and theoretical framework driving its development.

First, the chapter introduces the study context and supporting rationale behind its pursuit before discussing the NSSE benchmark derived theoretical framework and research questions. The research design process follows with specific reference to case selection, evidence triangulation, and ties to the theoretical framework. The chapter then details the research site, study participants, and qualitative field work procedures used over the course of the study. The next portion of the chapter explains the sources of evidence obtained during data collection and provides a detailed examination of the data analysis procedures employed. The chapter then shifts focus to participant protection and researcher positionality as primary considerations throughout design and implementation. Content throughout the remainder of the chapter discusses analytical rigor, specifically trustworthiness and study validity and reliability relative to the research design. A 
concluding summary is preceded by a discussion of study assumptions, limitations, and delimitations before introducing the findings chapter.

\section{Study Context and Rationale}

This study was conducted at a university located in Osh, the largest city in the southern KR. This region of Central Asia comprises the Ferghana Valley and has a rich cultural and political history as well as a multiethnic and multilingual population. From my perspective, the history and diversity of the region were reflected within the host university research site. It is a regionally prestigious public higher education institution that receives financial assistance and technical support from various external donor organizations and international governments (Issakov, 2014). The university and the foreign language teacher preparation program have participated in several quality improvement and higher education reform efforts (DeYoung, 2008; Merrill, Yakubova, \& Turlanbekova, 2015). Multiple accounts support that education in the KR remains primarily Soviet in nature, and that academic quality and student outcomes must improve for national competitiveness and stability (ADB, 2015; Brunner \& Tillett, 2007; D. W. Chapman et al., 2005; DeYoung, 2011). Researchers have specifically called for building professional capacity within Kyrgyz educational leadership and governance to foster the development and maintenance of higher education standards and practices and ultimately improve educational outcomes (Bayalieva-Jailobaeva, 2017; Cummings \& Nørgaard, 2004; DeYoung, 2008, 2011; Ryan, Tilbury, Corcoran, Abe, \& Nomura, 2010).

While completing a fellowship prior to this study, I observed many ineffective post-secondary education institutional policies, instructional practices, and student behaviors throughout the region. Although diminished academic quality throughout 
public education is a substantiated problem in the KR, the lack of effective and sustainable solutions is equally problematic (DeYoung, 2005). International donor agencies have repeatedly taken their own educational standards and practices and applied them in the KR, often without evaluating their efficacy or whether stakeholders agree to support and employ such changes (DeYoung, 2005). In the KR and throughout the postSoviet sphere, educators accept such impositions, at least at face value, to obtain much needed funding and sustain their livelihoods (Elliott \& Tudge, 2007b; Niyozov, 2006; Niyozov \& Shamatov, 2006). This study sought to explore practitioner and student perceptions via ethnographic case study to garner ground-level evidence to bridge the gaps between Western and post-Soviet higher education practices and standards in pursuit of improved educational outcomes.

This study took a preliminary step toward developing means for improving educational quality and outcomes for post-Soviet university stakeholders and practitioners. The exploration of perceptions of Western benchmarked practices in an institution within a post-Soviet context provides baseline evidence for potentially improving the quality and effectiveness of higher education throughout the region. Higher education research approaches the student engagement construct in various ways, from theoretical conceptualization to quantitative assessment, including the use of standardized survey instruments centered around research-informed practices (Kuh, 2009). To create a study that was appropriate for the scope of this analysis and the constraints of the research site while remaining potentially beneficial for stakeholders, the NSSE benchmarks were applied as the theoretical framework.

\section{Theoretical Framework}


The NSSE benchmarks of effective educational practice oriented this analysis around five key tenets of post-secondary education detailed in Chapter Two: an appropriate level of academic challenge, furthering active and collaborative learning, promoting enriching educational experiences, encouraging student-faculty interaction, and advancing a supportive campus environment ("NSSE Benchmarks," 2000). This analysis explored post-Soviet higher education experiences and practitioner perceptions of Western practices at the research site. Participants were placed at the center of the study and reflectively analyzed their own educational experiences and praxis relative to the benchmarks comprising the theoretical framework.

This study incorporated key theoretical propositions supporting the benchmarked practices of student engagement as detailed by Kuh (2002). As interpreted for this analysis, the NSSE propositions assert that: students' actions and experiences during university matter more than their background or the institution they attend, effective higher education practices yield student engagement, greater academic quality among post-secondary institutions is associated with high student engagement, and that measures of student engagement have practical potential within higher education reform (Kuh, 2002). Critically, the benchmarks were used as a frame for understanding how practitioners and students think about their teaching and learning and the potential for improvement in this context.

\section{Research Questions}

The main research question for this study developed from the fellowship experience and the extant literature on student engagement theory and practice. Subquestions focused on specific components of the main research question in describing 
practitioner and student experiences, defining effective educational practice, and analyzing the practical potential of the NSSE benchmarks. Additional sub-questions narrowed the analysis further by individually evaluating each benchmark and their aggregate perceived potential relative to praxis and higher education quality at the research site.

\section{Main Research Question}

How do post-Soviet practitioners reflect on their professional experiences and Western benchmarks of effective educational practice?

\section{Sub-Questions}

SQ1: How are practitioner and student higher education experiences described?

SQ2: How are effective educational practices among faculty and students defined?

SQ3: How are the NSSE benchmarks and their practical potential interpreted?

\section{SQ3 Sub-Questions}

SQ3A: How challenging is this academic program?

SQ3B: To what extent is learning active and collaborative?

SQ3C: In what ways do practitioners and students interact?

SQ3D: To what degree is the campus environment supportive?

SQ3E: How enriching are higher education experiences?

SQ3F: How might the NSEE benchmarks impact praxis and quality?

\section{Research Design}

This dissertation was initially proposed using an exploratory, embedded singlecase design and case study data collection and analysis methodology as defined by Yin (2017). The rationale behind this selection included the pertinence of the post-Soviet 
context, the contemporary nature of the research purpose and the uncontrollable nature of the research site (Yin, 2017). However, the focus of the dissertation evolved away from a program-level case study toward post-Soviet higher education practitioners, their professional experiences and practices, and their perceptions of Western practices.

This new analytical focus more closely aligned with and ethnographic research design and qualitative field work procedures focused on describing, analyzing and interpreting the behaviors, beliefs, and language of post-Soviet higher education practitioners (Creswell, 2008). The components, field research methods, and definitions of ethnographic case study emphasized by Creswell (2008) were observed throughout the design and execution of this dissertation as detailed in the Institutional Review Board (IRB) protocol (see Appendix A). As such, the ethnography could best be described as an instrumental case study as it consisted of an in-depth exploration of a bounded system to provide insight into an issue (Creswell, 2008). The case for this study became the learning and teaching environment within the foreign language teacher preparation program, and the embedded units of analysis emerged as the practitioners and students associated with the program.

The ethnography still included case study features as interpreted by Yin (2017) as it: 1) explored many variables associated with the student engagement construct; 2) included research questions derived from the NSSE benchmarks and relevant theoretical propositions; and 3) included multiple sources of evidence to triangulate data and contribute analytical rigor. However, key characteristics of ethnographic research predominated the study as cultural themes, culture-sharing groups, and shared patterns of behavior, belief, and language were emphasized throughout (Creswell, 2008). 
Case selection. The ethnographic case narrowed to the learning and teaching environment experiences within the foreign language instructor program. The embedded units of analysis within this case were higher education practitioners - faculty and administrators, as well as program students. This case was selected to both ensure accessibility, given my preexisting professional relationship with the program at the research site, and for practicality, given the limited financial resources available for conducting the study. Figure 1 in Chapter One depicts the case and embedded units of analysis examined at the research site within the institution.

Triangulation. Triangulating the experiences and perceptions within and across practitioners and students promoted credibility within the primary sources of evidence obtained through interview. The fidelity of findings was strengthened by incorporating evidence obtained from practitioners and students through semi-structured interviews and classroom observations via analytical comparison. In addition, member checks were performed by three participants: an administrator, a faculty member, and a student as a means of verifying study findings.

Member checks were performed at the conclusion of data collection and preliminary analysis during the composition of Chapter Four prior to the interpretation and discussion sections of Chapter Five. I asked three participants who had expressed interest in observing the final outcomes to review preliminary findings and gauged their responses. Due to logistical constraints, the member checks had to be conducted via WhatsApp messaging and phone calls, although individual interviews would have been preferred. My objective in obtaining participant responses to the preliminary findings 
centered around fostering greater qualitative rigor prior to composing the discussion and interpretation elements in the final chapter.

The member check lines of questioning stemmed from a qualitative trustworthiness framework developed by Billups which built upon standards of quantitative research rigor (2014). When each member was asked why they volunteered their time to assist in member checking they stated that they did so out of general interest, to assist in the completion of the study, and to help me as a personal favor. The faculty participant responded with, "I just wanted to share what is true in our country" (Participant 01, personal communication, June 15, 2018). As applied within this study, I had the three members check my initial findings for quantitative validity and qualitative credibility, by asking them if they found the findings believable and truthful (Billups, 2014). When pressed for further explanation, I explained to each member that I wanted to know whether they thought that the findings were correct in their opinion and from their perspective. All members supported that the findings were believable and that they represented the truth within this specific higher education learning environment.

To strengthen the study in terms of quantitative reliability and qualitative dependability, I asked the members whether the findings would be similar if the study were repeated over time (Billups, 2014). All members supported that the findings would most likely be similar if the study were repeated after one or five years, with potentially major differences after ten. For qualitative transferability and quantitative generalizability, I asked whether the findings would apply to other universities in the KR and post-Soviet countries in general (Billups, 2014). The administrator and faculty member thought that findings would be similar for most universities in the KR; they 
doubted whether results would be the same in other countries, even those in the region. The student member, who had attended another university in Bishkek, stated that their experience there differed greatly. They anticipated that findings for student experience and learning would most likely skew negative if the study were repeated in the north.

To assess whether findings were neutral, I pursued qualitative confirmability and quantitative objectivity by comparing and contrasting participant responses and by analyzing the member check responses (Billups, 2014). I found a greater degree of continuity and agreement between the administrator and faculty members compared to the student member. However, their perceptions of the initial findings tended to support one another as opposed to running counter to each other. Finally, to advance tenets of qualitative authenticity and quantitative accuracy, I asked each member whether the reality of being a student, a faculty member, or an administrator were represented within the preliminary findings (Billups, 2014). Members struggled to understand this aspect of the framework until I rephrased the question as, "is your story told in these findings?" The student member stated that their experience was represented, the administrator largely deferred but said that their time at work was present, and the practitioner said that the story of their experience was honestly portrayed by the study.

Ties to the theoretical framework. A checklist for theoretical framework integration developed by Cynthia Grant and Azadeh Osanloo (2014) clarified and substantiated the role of the NSSE benchmarks within this dissertation. As a study of teachers and learners within a post-Soviet higher education institution, the ethnographic case study design complemented the cultural, contextual, and environmental components of student engagement underlying the theoretical framework well. Specifically, the 
qualitative field research methods espoused the collection and analysis of multiple sources of evidence necessary to investigate the multidimensional nature of the overarching student engagement construct (Creswell, 2008; Kahu, 2013; Yin, 2017). In addition, tenets of reflective analysis and theories of practice were incorporated into the development of the semi-structured interview protocols to specifically target the evidence-based practices associated with NSSE (Argyris \& Schön, 1974; Schön, 1983). The theoretical framework is of a scope sufficient to address the post-Soviet higher education topic and study objectives, particularly the collection and analysis of evidence to gauge practitioner professional experiences and perceptions of Western practices.

The research problem, that Western practices have failed to evince change within post-Soviet educational praxis or institutional academic quality correlates with the overall intent of the NSSE benchmarks: to inform higher education institutions about their students' experiences and guide efforts to improve academic performance and educational outcomes (Kuhn, 2002). The research purpose, gathering practitioner experiences with and perceptions of student engagement practices was supported throughout. The significance of the study is reflected in the increasing prevalence of the student engagement construct in evidence-based practice and within international higher education research and practice (Coates \& McCormick, 2014; Fredricks et al., 2004; Kahu, 2013; Zepke, 2014). Iterations of the main research questions and sub-questions targeted aspects of the student engagement construct and the individual NSSE benchmarks within the research site context. In addition, the interview protocol item rationales (see Appendices B and C) for the semi-structured interview protocols (see Appendix D) traced individual benchmarks through each item. 
The theoretical framework focused the preceding literature review on the body of higher education research pertaining to student success and the development of the student engagement construct as well as the benchmarks themselves. The qualitative data analysis and coding plan allows for the development of grounded codes that could be connected to or potentially refine the existing NSSE benchmarks. Finally, the discussion, implications, and conclusion sections were developed with direct reference to the theoretical framework and its role within higher education practice and research.

\section{Research Site}

The Kyrgyz government officially established a university system in Osh in 1992, but many of the higher education institutions in this system trace their origins to Soviet pedagogical institutes established in 1951 (Issakov, 2014; "Osh State University," 2017). The host university for this study is considered the most prestigious in the region and ethnic Kyrgyz and Uzbek students with Kyrgyz nationality are permitted to enroll on the basis of national scholarship testing, with selected students receiving a stipend and paying no tuition (Participant 01, personal communication, November 15, 2017). In addition, students of all ethnicities and nationalities are permitted to enroll via selffinanced tuition contracts and the international student populations includes learners from India, Pakistan, Tajikistan, Uzbekistan, and Turkey, among others (Participant 01, personal communication, November 15, 2017). As an external researcher building upon evidence obtained from my fellowship and observations during the study, while leadership at the research site was clear at the program level with senior instructors and a dean; higher levels of the administration, bureaucracy, and leadership were notably opaque and subject to change with little notice or explanation. 


\section{Participants}

This study relied on evidence obtained from volunteer participants: practitioners and students within the case. The foreign language students, faculty, and administrators associated with the teacher preparation program volunteered to contribute to this dissertation based on our interactions throughout the fellowship and ongoing communication and consultation. Two cohorts of university practitioners and students were included as participants, a sample size in line with established qualitative interview research standards (Crouch \& McKenzie, 2006; Guest, Bunce, \& Johnson, 2006). As part of the inclusion criteria for this study, all participants needed to have appropriate levels of English language fluency, accuracy, and proficiency to be able to understand the study content and interview questions. I purposefully included student volunteers to garner additional evidence and perspective on the educational experience within the case and to corroborate practitioner perceptions. This inclusion of student voice also demonstrated my commitment to student participation, transformation, and empowerment within this specific higher education context (Seale, 2010). To garner additional information regarding the context of the case, I triangulated data both within and across participants as well as my own classroom observations, this technique helped to interpret, situate, and validate evidence obtained.

\section{Field Research Procedures}

Cresswell (2008) defined fieldwork as gathering data in the setting where participants are located so that their shared patterns including behaviors, beliefs, and language can be studied. By incorporating adaptive procedures as approached by Yin (2017), I was able to conduct the analysis in this highly variable, remote context where 
changes in the case and availability of evidence were inevitable, especially in my role as an external researcher. Yin (2017) explained adaptive design as alterations and modifications to an original case study that incorporate revelations uncovered by new information obtained during data collection. Importantly, I took care to ensure that I accommodated revelations without sacrificing analytical rigor or impacting ethical considerations, and without narrowing the scope of the study at the expense of more comprehensive exploratory evidence. When assumptions or preliminary research were contradicted by participants or other evidence, aspects of the analysis were altered to be inclusive of such revelations while maintaining the existing protocol. Case in point, participants required significantly more time to review the NSSE benchmarks in order to comment on them effectively, so the time in between interviews had to be extended. In addition, where logistical or practical constraints impeded the study, such as university closure, difficulties were noted and accommodated without raising additional ethical considerations. For example, difficulties with coordinating follow-up interviews with some practitioners led to four not being fully included in the study as initially planned. Also, successfully locating volunteer participants with satisfactory English language skills proved to be a challenge as some interview content consisted of single word responses or had little correlation to actual interview questions. This presented an opportunity to gain richer data from within the existing semi-structured instrumentation via further probing and scaffolding within purposefully selected individual interviews. Finally, given the known technological limitations at the research site, data loss due to power outages were anticipated and accommodated for by uploading information as soon as possible and securing it within an encrypted database. 
A university official from the program case granted permission to conduct this study as I had previously completed a fellowship at the host university. The IRB study protocol (see Appendix A) and preamble (see Appendix G) were reviewed by the administrator prior to data collection. In return for access to the program, I agreed to conduct International English Language Testing System/Test of English as a Foreign Language (IELTS/TOEFL) courses for students and faculty, assist students and faculty with international exchange applications, consult administrators about proposed program changes, provide professional development opportunities to faculty, and provide a case study report to the administration for review as well as the NSSE benchmarks being investigated (see Appendix E).

\section{Data Collection}

The purpose of this qualitative study was to explore practitioner perceptions of Western higher education practice from a post-secondary institution located within a post-Soviet context. Thereby, the data collection process was designed to gather evidence to understand how higher education practitioners in the KR reflect on experiences, praxis, strategies, and outcomes in their program relative to the NSSE benchmarks. Finally, evidence associated with the student engagement construct and NSSE were purposefully included to focus instrumentation development and my own observational and reflective data on the core of the study. Creswell (2008) differentiated between fieldwork data and stressed the importance of obtaining information directly from participants (emic data), information representing the ethnographer's interpretation of the participant's perspectives (etic data), and negotiation data or information that the participant and the researcher agree to incorporate (p.471). The intent of data collection 
was to generate saturated qualitative emic, etic, and negotiation data from multiple participants embedded within the case which could then be analyzed.

Data collection also followed the principles detailed by Yin (2017) by: 1) locating multiple sources of evidence, in this case forty practitioner and student participants; 2) creating a case study database, the interview transcripts uploaded to a secure cloud; 3 ) maintaining a chain of evidence, matching participants with their transcribed interviews via order number; and 4) being cautious with external sources of evidence by only including participants within the case under analysis. All data collection complied with qualitative ethical and methodological standards and the IRB approved study protocol and an overview of the total data collection process is provided (see Figure 5).

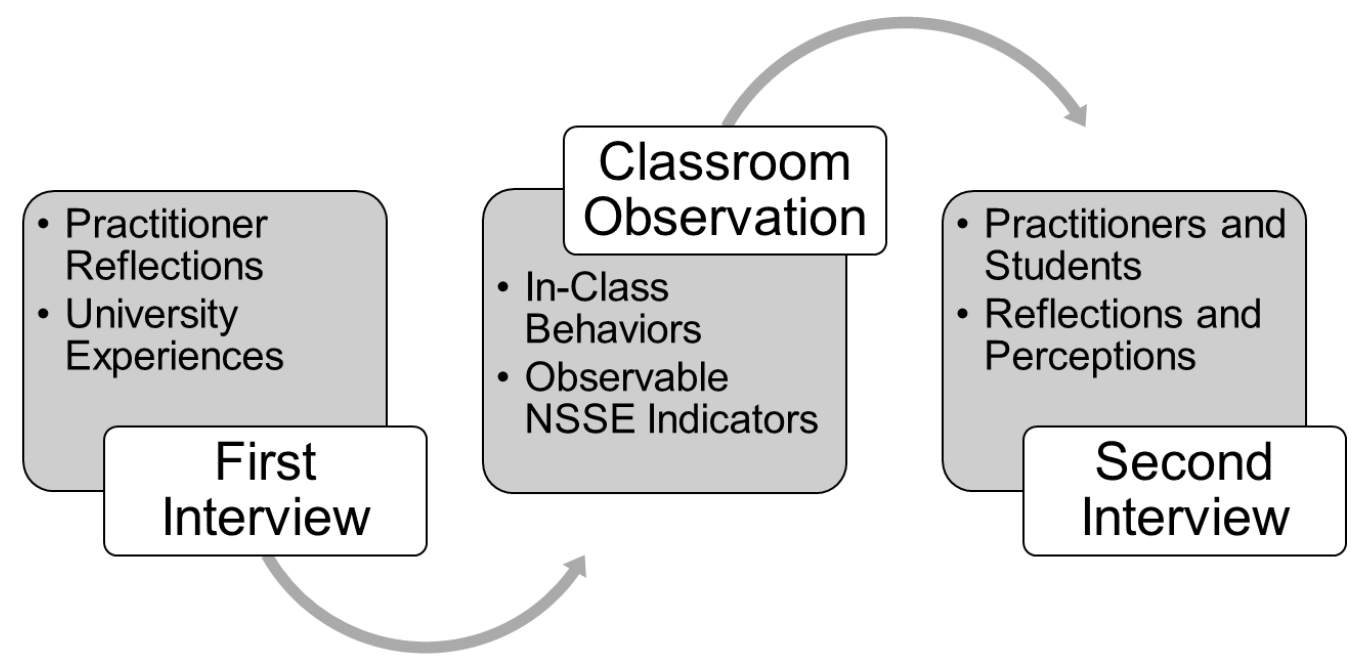

Figure 5. Study Data Collection Procedure. This figure depicts the three-step process of data collection employed in the study to obtain information from interviews and observations.

Interviews. Semi-structured interviews generated transcripts of reflective content exploring participants' perceptions of the NSSE benchmarks relative to their praxis and the program. I employed the semi-structured interview protocols in a series of one-hour 
interviews targeting practitioners and students (see Appendix B). The first round of interviews focused on practitioner reflections concerning their university experiences both as a student and as a teaching professional. The second round of interviews pertained to both students and practitioners and sought to identify their reflections on university experiences and practices using the NSSE benchmarks as a theoretical lens, as well as their perceptions of the benchmarks themselves. Participants' interest and willingness to volunteer was largely dependent on their availability and English language abilities, as it was essential that they could handle relatively complex interview subject matter. Participants received the protocols and associated content on paper and via WhatsApp, including the published NSSE benchmarks, several days before their respective interviews so they could prepare accordingly. Prior to each interview, participants were provided a hard copy of the study preamble and a verbal reminder concerning the voluntary nature of their participation and the recording, transcription, and analysis of the interviews.

Direct observations. I used an adapted classroom observation instrument (see Appendix F) to center field notes around observable indicators of the NSSE benchmarks with emphasis on instructional praxis, student behaviors, and institutional features. Given the very small classrooms most instructors have within this institution, it was necessary to be very discreet while completing the instrument. Luckily an early study participant revealed that classes are often observed by senior instructors and administrators, so my presence was relatively unobtrusive to most classes (Participant 01, personal communication, November 15, 2017). 
Instrumentation. As English is not the lingua franca within this region and was the third or fourth language e for some study participants, I used simple language in all instrumentation for the content to remain as accessible as possible. Multilingual interpreters from the research site volunteered their services to alleviate some linguistic difficulties during instrument development. Unfortunately, the additional complexity and time requirements associated with having volunteer interpreters present during interviews was deemed infeasible during study development. As such the semi-structured interview protocols included multiple lines of questioning around the research question and subquestion foci, garnering rich, saturated data from participants to subsequent for transcription and subsequent analysis. I maintained hard copies of all documents to avoid complications from the frequent electrical outages, technological failures, and general lack of technical literacy at the research site. The direct observation instrument aided data collection by providing a simple representation of the NSSE benchmarks and their observable behaviors and features. I worked with non-participants to design and pilot and refine all instrumentation prior to obtaining IRB approval and conducting the study.

By employing a generalized participant interview protocol, I was able to tailor items to each individual interviewee, thereby stressing the exploration of perceptions of the NSSE benchmarks relative to program praxis, focusing discussion on the impacts of the research site context, and keeping reflective interview content centered on university experiences and perceptions. The interview protocol item rationale table (see Appendix B) linked each interview item to corresponding elements from the research questions and theoretical framework. The interview protocol assisted in exploring practitioner and 
student perceptions through reflective analysis of their praxis and learning experiences relative to the NSSE benchmarks.

\section{Data Analysis}

Tenets of ethnography including the collection of emic, etic, and negotiation data to yield a rich description of the culture-sharing group which in turn contributes to the generation of themes and a conclusive interpretation (Creswell, 2008). Data analysis for this study centered around merging and investigating the evidence obtained into ethnographic findings via data reduction/condensation, data display, and conclusion drawing and verification (Berkowitz, 1997; Miles, Huberman, \& Saldaña, 2014). By combining ethnography with qualitative content analysis, a unified structure for the processing and interpretation of data study data resulted (see Figure 6).
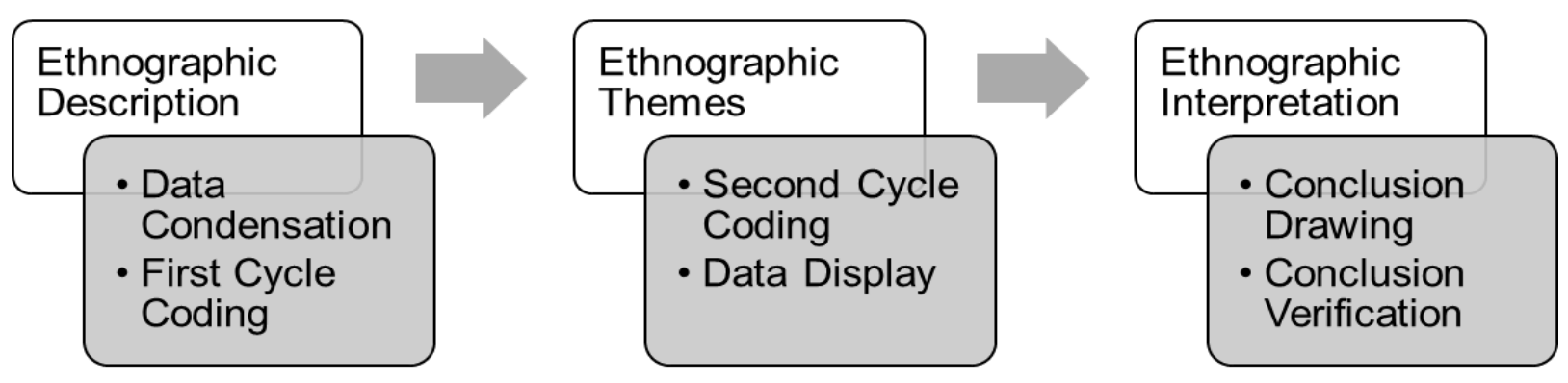

Figure 6. Ethnographic Overview and Qualitative Content Analysis Procedures.

This figure indicates the objectives of the ethnography and the relevant qualitative content analysis procedures for generating those outcomes. 
All interviews were transcribed as rapidly as possible given the potential for data loss at the research site due to technological constraints and the potential benefits of adaptive procedures obtained from concurrent analysis and data collection (Miles et al., 2014). The generation of an ethnographic description of the context of the research site and participants served to situate the analysis of the interview data and contribute to the application of the theoretical framework in generating codes. Analysis of the transcribed data took place via First Cycle and Second Cycle coding procedures as outlined in Figure 7 (Miles et al., 2014; Saldaña, 2015).

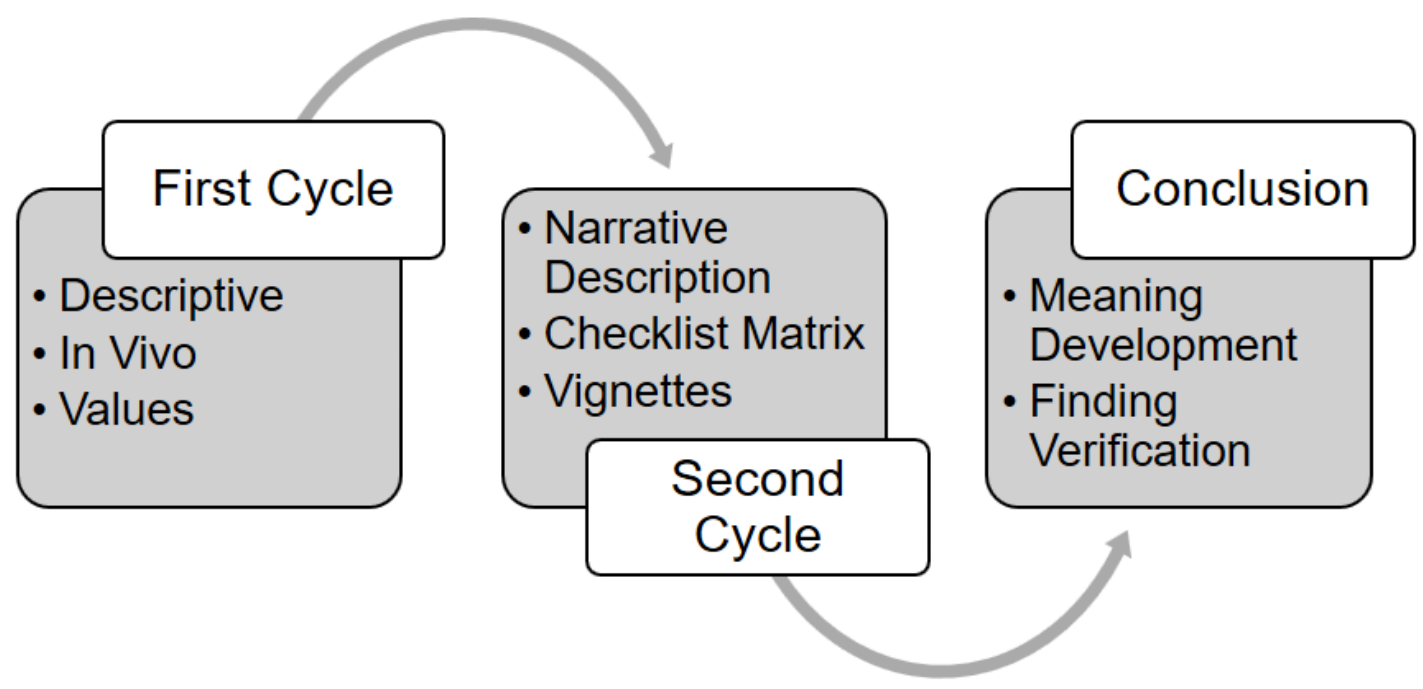

Figure 7. First Cycle and Second Cycle Coding Procedures and Outcomes. This figure depicts the coding procedures used throughout the iterative processes of data collection, condensation, display, and conclusion drawing and verification.

First Cycle Coding Procedures. Initial coding procedures consisted of descriptive coding to index and categorize interview data into an inventory, in-vivo coding to use participants' own language as codes, and values coding to reflect each participant's world view as described by their values, attitudes, and beliefs (Miles et al., 
2014). Saldaña (2015) defined values and attitudes, respectively, as the importance attributed to versus the way people think about themselves, people, things, or ideas (p.298). Beliefs are defined as a constructed system of values and attitudes, personal knowledge, experiences, opinions, and perceptions of the social world (Saldaña, 2015, p.298).

Second Cycle Coding Procedures. Pattern coding functions primarily in the second coding procedure to condense data into smaller units, in this case ethnographic themes and explanations pertaining to the post-Soviet university experience at the university under analysis (Creswell, 2008; Miles et al., 2014). It also functions to center the researcher on analysis during data collection and aids in the development of integrated schema for understanding practices and interactions under investigation (Miles et al., 2014). In this study second cycle coding consisted of three discrete parts: a narrative description of the ethnographic pattern code themes, a checklist matrix of participants' perceptions of the NSSE benchmarks, and vignettes capturing significant fieldwork moments (Miles et al., 2014).

Drawing and Verifying Conclusions. The application of the various data analyses employed in this study are ultimately used in the development of meaning and verification of findings and conclusion via analytical tactics (Miles et al., 2014). Specific tactics employed for conclusion development and verification purposes, including member checks and other techniques to support qualitative rigor, are discussed throughout the final chapter in the context of the data to which they were applied.

\section{Protection of Participants}


The successful completion of this study depended on effort and input from volunteer practitioner and student participants who face major time constraints and financial difficulties in their daily lives. Given that this research occurred during an election year, and that the southern region has historically been the epicenter of ethnic and racial tensions, confidentiality and non-disclosure were essential. To avoid entanglement in the extensive corruption and security issues at the research site, I emphasized that I would not pay any bribes or provide any gifts in exchange for case access to relevant gatekeepers. In the interest of the beneficence of participants, informed consent was obtained via unsigned preamble, participant names were not collected, and contact information and recordings were only linked to participation order numbers for organizational purposes to further protect confidentiality. Participants were consistently reminded throughout the data collection process that their participation was voluntary and that it was perfectly acceptable if they chose not to answer a question or participate at all. I restricted access to all identifiers by only maintaining such information on an encrypted laptop, mobile device, or secure cloud database in line with IRB requirements. In addition, all identifiers were destroyed at the conclusion of the dissertation. I assigned pseudonyms or used participant numbers to reference individuals and other entities that requested anonymity. Participants were encouraged to reach out to me with any concerns or study-related requests via WhatsApp, email, or phone (my contact details were provided to all participants).

\section{Researcher Positionality and Reflexivity}

The teaching fellowship that led to the development of this study altered my perspective and I am grateful for that opportunity. I personally would never have had the 
impetus to travel to the KR, much less spend a year working and living there.

Throughout the course of this study, my position as a former fellow (and current researcher) impacted my perceptions of experiences and events. I began to make sense of aspects of life, work, and education in this region that were completely foreign at the beginning of the fellowship over two years ago — now, my background in the KR has taught me that this is simply how many things are done in this region. With my position as a researcher in this study, I acknowledged that aspects of myself and my background may have impacted some interpretation of the empirically-based information obtained. Aspects of objectivity and subjectivity are, to varying degrees, intrinsic components of researcher interpretation (Alexander, 2017; Hegelund, 2005). Some ethnographic aspects of this analysis appeared to defy impartial and objective explanation. Reflexivity, the process of continually assessing positioning via internal dialogue and critical selfevaluation, is essential to maintaining the integrity of qualitative analyses (Alexander, 2017; Berger, 2015; Creswell, 2008). Reflexive practices were maintained through introspective journaling and maintaining reflective practice throughout the dissertation process.

I have shared experiences with the faculty in this case as I taught some of the same students within the program in years prior. My roles have also shifted, from a foreign consultant (outsider) to a member of the university faculty (insider) to a doctoral candidate researcher (outsider once more). I have received respect and privileged treatment from participants in this study simply because of my social position as a white male American graduate student, and I recognize that this treatment most likely has much more to do with their cultures and practices than my own. As such, study participation 
was most likely volunteered by participants out of kindness and respect rather than genuine interest. Throughout the fellowship and this study, my negative and cynical biases manifested in my interpretations of, perceptions of, and beliefs about almost everything being attempted. I focused on thinking critically rather than being critical in approaching ethnographic case study analysis. At the outset, I lacked an understanding of the KR's developmental history and of the internal and external forces impacting the university. My failure to critically reflect on my own personal higher education experiences certainly contributed to this, and such insight led to regret and some animosity. In reflecting on this negativity, it became apparent that while I was born into a nation with tremendous wealth and the semblance of opportunity, the United States faces many of the same practical issues and unfortunate realities I explored in this dissertation and is just as likely to prove resistant to change.

My positioning as a researcher throughout this study had impacts upon both the data obtained and the final findings. As noted previously, my presence in classrooms for observations was noted by practitioners and participants, with some actively involving me in their lessons. While I intended to simply observe and document the classroom learning environment, my presence in the classroom certainly impacted observational data as observing while simultaneously documenting proved to be challenging. Out of respect I opted not to type into the classroom observation instrument while engaging in conversation or classroom discussion. As a token of respect and appreciation, I opted to complete the instruments at the end of classes if practitioners and student chose to engage me in the lesson of the day. Throughout these lessons, the topic of my nationality arose 
quite frequently in conversation, and while no one was hostile or negative, it did feel like its discussion highlighted the substantial divide between myself and participants.

The divide between myself as a researcher and participants in this study was most evident during the two rounds of interviews. In reflecting on their higher education learning experiences, practitioners frequently remarked, "this is our culture," relative to actions such as respecting elders unequivocally and exhibiting deference toward experts and specialists. In reflecting on this remark, I interpreted it as also indicating that such practices were not within the bounds of my culture. Further dialogue could have revealed more commonalities than differences between my culture and those of participants. For example, when participants were asked why students keep quiet despite corruption or tolerate blatant injustices, I viewed this as a commonality with my own experience and culture. I interpreted this practice as being symptomatic of a lack of student empowerment both in my own higher education experiences and in that under analysis in the KR. From my perspective, students and practitioners must be empowered to recognize that culture cannot be the default response when organizations do not function properly, and stakeholders are disenfranchised.

While differences exist between my culture and that of the participants in realms including politics and religion as well as cultural practices concerning marriage and family, our common beliefs and values in education are worth highlighting. Practitioners and students in the KR readily admitted that the quality of public education at all levels throughout the country needed to improve. This call for improvement echoes those of educational reformers, trusts, and foundations throughout the United States who lament declining academic performance and less than desirable student outcomes. From my 
position, the educational contexts of both the research site and higher education in the United States could benefit by adopting a continuous improvement perspective. I argue that this study presents evidence that attempting to find the optimal solution to an educational problem rather than striving to improve practice along the way can be just as deleterious as doing nothing to students and organizations alike.

\section{Trustworthiness}

The divides between researchers and practitioners from and within the disciplines bring into question the legitimacy and value of divergent research paradigms and practices. The pursuit of rigor in research takes many forms under many conditions; Billups asserted that "rigor in any qualitative study ultimately resides with the quality of the researcher's purpose and practice, and the verity of the unique depth and breadth of each participant's 'lived experience'," (2014, p. 4). This study captured the lived experiences of post-Soviet higher education through the perceptions and reflections of study participants, analytical rigor was supported through multiple means. I first discuss ethnography design and methodology considerations for validity and reliability before approaching components of qualitative trustworthiness according to Lincoln and Guba (1985) consisting of credibility, dependability, transferability, and confirmability as well as authenticity as interpreted by Polit, Beck, and Timmins (2013). A comparison of quantitative and qualitative means for supporting analytical rigor as adapted from Billups (2014) and Miles, Huberman, and Saldaña (2014) is presented in Table 1 along with specific means employed in this study before being individually detailed in separate sections. 
Table 1.

A Comparison of Qualitative and Quantitative Means for Promoting Analytical Rigor with Study Application.

\begin{tabular}{lll}
\hline Quantitative & Qualitative & Study Application \\
\hline Validity & Credibility Authenticity & Triangulation \\
\hline Reliability & Dependability Auditability & External inquiry audit \\
\hline Generalizability & Transferability Fittingness & Thick description \\
\hline Objectivity & Confirmability & Audit trail \\
\hline Accuracy & Authenticity & Member checking
\end{tabular}

Note. Adapted from Billups (2014) and Miles, Huberman and Saldaña (2014).

Ethnographic Case Study Validity and Reliability. Contradictions in basic

institutional data reflect the difficulties of conducting quantitative research in this region although a general lack of reliable and valid data is not unique to higher education in the KR. Researchers throughout Central Asia struggle with inaccessible, invalid, and unreliable data, Landau and Kellner-Henkele stated: "Moreover, the profusion of sometimes contradictory information leads to confusion. Relying on such data unreservedly can be precarious," (2011, p. 200). The plausible manipulation of data for political gain was effectively summarized by Dave (2004) "What the state had failed to achieve on the ground has been attained through statistics," (p. 455). The quality of international education statistics, particularly those employed by the United Nations Educational, Scientific, and Cultural Organization (UNESCO), have led to several critiques and calls for reform, especially when such statistics are essential for assessment and decision-making processes (Cussó, 2006; Heyneman, 1999). 
By approaching from a qualitative research perspective, I accounted for the questionable validity of existing quantitative higher education data at multiple levels. By focusing on the experienced realities and perceptions of practitioners and students within the foreign language pre-service teacher program, I restricted the embedded units of analysis to practitioners and students in the learning and teaching environment case.

Credibility. This dimension ascertains the extent to which findings are believable, truthful in representing reality, and inclusive (Billups, 2014). In the context of this study credibility was reinforced through prolonged engagement, as I worked at the research site for one academic year and have remained involved by proxy for more than two. Persistent observation was reinforced throughout as the post-Soviet context and exploration of situated practitioner experiences and perceptions guided the study. Peer debriefing was included by having a doctoral student compare conclusions to address bias, factual errors, alternative interpretations, data convergence, and themes. Memberchecking was applied in that three key study participants were asked to review the preliminary study findings to assess whether what they expressed was included and interpreted properly. Triangulation was incorporated within data by including multiple practitioners and study participants and corroborating their experiences and perceptions, by applying negative case analysis by investigating contradictory evidence within the study, and in theory by the inclusion and application of the NSSE benchmarks (Billups, 2014).

Dependability. To determine whether findings stemming from the research process were consistent and stable over time, an external inquiry audit was conducted with an independent researcher reviewing the case study chain of evidence, findings 
(Billups, 2014; Miles \& Huberman, 1994). The degree of fit between the external inquiry audit and the case study was analyzed and interpreted within the findings.

Transferability. Whereas case study internal validity and qualitative credibility were strengthened by the breadth of data sources incorporated, transferability and ethnographic case study external validity were supported by the depth included within each data source (Billups, 2014). In line with Geertz's (1973) strategy of thick description, instrumentation was developed to extract and include extensive detail to ascertain whether the research might transfer under similar study conditions.

Confirmability. Confidence of accuracy within the study findings was furthered by supporting conditions for study replicability (Billups, 2014; Miles et al., 2014). The IRB research protocol describes study procedures and methods in sufficient detail to be audited externally, findings are presented sequentially, and personal assumptions, values, and biases are addressed specifically (Miles et al., 2014)

Authenticity. The representation of multiple realities to provide the meaning of the findings promoted this final aspect of trustworthiness (Polit et al., 2013). By identifying the contextual purpose of the research, its intended value, and how the research benefits participants, the ultimate goal of the study was further assured (Billups, 2014).

\section{Assumptions, Limitations, and Delimitations}

This section details the methods and procedures used to accommodate and mitigate assumptions, limitations, and delimitations as described by Simon and Goes (2013) within this study. The post-Soviet nature of the KR and the realities of operating a higher education institution within the complex, changing, and unstable context 
challenged my ability to conduct this study at the research site. By detailing the assumptions, limitations, and delimitations inherent to the development and execution of the dissertation, the trustworthiness, validity, and reliability of evidence obtained is further supported.

Assumptions. The reflective analysis of university experiences and the NSSE benchmarks comprised the core of this dissertation. This required developing research protocols with reflective lines of questioning for practitioner and student participants with little exposure to study content or interview procedures. Language represented a continuous challenge throughout this study, a foreseen issue as effective communication was problematic throughout the fellowship. Given the complexity of discussing Western benchmarked higher education practices, linguistic selectivity for case practitioners with adequate English skills within the university program was necessary. As such, volunteer practitioner participants with interpretation and translation experience and students with developed English fluency and proficiency were highlighted. To ensure that participants felt comfortable voicing genuine opinions of the case, I conducted interviews away from the university and emphasized the confidential nature of the preamble and IRB protocol.

The university program at the research site was not a data-driven enterprise with carefully collected and maintained databases or an office of institutional research. Accessible existing data were of debatable accuracy and quality. The research site context embodied conditions of complexity, instability and uncertainty which posed numerous methodological difficulties. These challenges, among others, led to the selection of the ethnographic case study design and the inclusion of adaptive technique as a means of maintaining analytical objectivity. Contradicting evidence still proved 
frustrating as study participants and available sources of data purported to report definitive truths concerning student experiences, outcomes, and performance, yet diverged greatly. This was accounted for by triangulating sources of evidence throughout data analysis and interpretation as a means of promoting analytical rigor and strengthening findings. Finally, it was assumed that practitioner perceptions would be revealed over the course of the analysis and that these perceptions would only be analytically generalizable in accordance with tenets of case study design (Yin, 2017).

Limitations. The qualitative ethnographic case study design and research methods included limitations regarding the study and its findings, especially causality and generalizability. Specifically, as a case study, findings were only generalizable to theoretical propositions and not to populations (Yin, 2017). In addition, sources of evidence were restricted within the research site to interviews with practitioners and students and classroom observations, restricting the scope of data source inclusion. Study participants including some foreign language program faculty members were found to possess highly variable English language abilities. To accommodate this, challenges during interviews were noted, participation was encouraged among a greater number of practitioners, and extended follow-up procedures for clarification of interview content were added. Extensive corruption, cultural differences, and language barriers in the case may have impacted participation and responses despite preemptive agreements being made with the university to lessen any potential impact.

Delimitations. Given the nature of the research site context, it was necessary to limit the scope of the analysis and intentionally choose to exclude or include certain parameters. Case in point, the study was designed to explore practitioner perceptions of 
the NSSE benchmarks rather than the NSSE itself as it was too complex, expensive, and generally infeasible to translate associated instruments to the research site. In addition, the original benchmarks were selected over the current NSSE engagement indicators and high-impact practices given the exploratory nature of the dissertation, its practical versus conceptual focus, and the comparative linguistic simplicity of the benchmarks. The scope of the analysis was restricted to a case study of the teaching and learning environment within a single post-secondary teacher preparation program in the KR. Phenomenological and grounded theory qualitative methodologies would have limited the application of relevant theory, contributing greater complexity and length to the study without justification for doing so. The adaptive nature of ethnographic case study design compensated for the changeable nature of the research site and accommodated participant interview and classroom observation information while situating data analysis and interpretation within relevant student engagement theory.

\section{Summary}

This study used an ethnographic case study design and qualitative field research methods to explore practitioner perceptions of the potential of Western evidence-based practice in a higher education institution in a post-Soviet environment. The adaptive design enabled me to draw from multiple participants as sources of evidence to support analytical rigor while maintaining relevance to the program and study objectives (Schön, 1983; Yin, 2017). I combined the strengths of ethnography and the data collection approach with systematic qualitative content analysis to yield findings centered around tenets of validity and reliability while accommodating my positionality and study 
limitations. In Chapter Four, I present findings from each stage of the study and detail information obtained from their analysis prior to interpretation. 


\section{CHAPTER IV}

\section{FINDINGS}

This study aimed to explore the experiences of post-Soviet higher education practitioners and their perceptions of Western student engagement practices. I incorporated tenets of reflective analysis throughout study development, implementation, and analysis. This chapter details the study findings, beginning with an analysis of the sociocultural context in which it was conducted. This is followed by an ethnographic description of the individuals, scenes, and groups within the study, this was used to focus on the extent to which cultural contexts were relevant to findings. Next, I explore information from the first round of interviews, classroom observations, and second round of interviews. This section encapsulates the data condensation phase of analysis; I include descriptive, in-vivo, and values-based information from first cycle coding. I then present the ethnographic themes derived from second cycle coding. I detail data display by providing a narrative description, a student engagement content-focused checklist matrix, and selected participant vignettes to fully illustrate the data obtained from the research process. In the concluding section of the chapter, I present findings specific to each research question, reserving their discussion and interpretation for Chapter Five.

\section{Sociocultural Context}

The findings from this study must be interpreted from a perspective including the sociocultural context from which they were obtained. The value of the study findings 
extends from their collection at a research site and practical context which differs significantly from the theoretical context from which the benchmarks originated. Throughout the presentation of findings in this chapter and their discussion and interpretation following, cultural context is stressed as the means for weaving together multiple sources of evidence into the overall findings. As such, cultural aspects running throughout the remaining chapters are defined directly.

Fully describing the sociocultural context at the research site in Osh could encompass an entire dissertation. Through the processes of data condensation and display discussed throughout the remainder of this chapter, it was my intent to portray the external and internal forces impacting the ethnographic case. In doing so, the findings can be viewed as observations and reports of cultural practices taking place within the research site and the teaching and learning environment under investigation.

\section{Ethnographic Case Description}

Along the walk to the university, the city of Osh appears to simultaneously represent its ancient history, Soviet past, and post-Soviet future. Mountain ranges overshadow the city and dwarf the identical housing blocks that stretch into the distance. Soviet facilities, including the philharmonic, concert hall, and library, epitomize brutalist architecture. And yet, they clearly are falling into disrepair-cracked gray concrete and cold broken marble lie on every surface. The harsh winters coat everything in icy black sludge, and the few crowded walkways are hazardous and in disrepair. Pipes wrapped in asbestos comprise the city's central heating system, hanging over sidewalks and crossing the open drainage sewers next to each road. Smoke from coal-burning home furnaces and power plants engulfs the streets and buildings, creating a fog that lingers for days. 
The open-air bazaar is the ancient heart of the city; traders sell goods and produce from a chaotic patchwork of reclaimed shipping containers and whatever else can be found and recycled in use. A multitude of cars, public-transit vans (marshrutkas), and families with children jam every available sidewalk and street. It is at once exciting and unsettling.

The university itself sprawls across the city. Buildings painted a glaring red with white trim stand out from the desolate gray of the winter months. Tall iron gates and meticulously manicured gardens surround each department's miniature campus, which consists of a main building, a large reception or theater, and always an outhouse. Billboards with pictures of recent graduates and faculty wearing stern, hostile expressions adorn the main buildings alongside banners announcing ongoing admissions. The department studied in this case is housed in a former dormitory for the main university, though the administration promised a new building decades ago. A library is housed underneath an auditorium; its computer labs are non-functional, and students visit infrequently. Inside the university's gates, students huddle in small groups. Most students in attendance are female, and they dress in the brightest colors imaginable with the nicest boots they have, somehow shining and spotless despite the winter slush. Students approach and question foreign newcomers with a kind but harsh manner asking: "How are you? How old are you? Where are you from? What do you do? Why are you here?" in a never-ending cycle.

In the department's main building, students line the hallways, stairwells, and any available areas, waiting for classes and just standing around. Elderly women wearing dark heavy dresses and brightly patterned kerchiefs to cover their hair shoo students away while constantly sweeping and mopping the slippery concrete floors and stairways. 
Aside from a main lecture hall and two conference rooms, classrooms are no larger than 200 square feet. They resemble dorm rooms but with bench seating filling every available area, a small desk for the instructor, and a small whiteboard or unusable blackboard on one wall. Technology is limited to a single fluorescent lighting fixture and a few electrical outlets located haphazardly around the walls. Echoing voices from the hallways bounce around these classrooms, and class is interrupted by a steady stream of administrative assistants taking attendance, late students, lost students, faculty with questions, and friends just dropping by.

Walking past the classrooms on each of three floors, consistently there are students but no faculty. In this department, almost all faculty are female, and the majority graduated from this institution under the guidance of current administrators. Instructors are of Kyrgyz, Russian, and Uzbek heritage, and they are thoroughly professional in attire and reserved in demeanor. Faculty members speak English with a distinctive British accent, resulting in a dialect that is distinguished by formal, archaic vocabulary and an overemphasis on pronunciation. Faculty in the building are kept busy as administrators constantly shepherd them between meetings in various rooms or call them to other duties. Administrators remain in their offices to meet with faculty, parents, and the occasional student, and all offices feature external cameras that alert the administrators about their next appointments. Relationships appear to be cordial between faculty and administrators; they exchange pleasantries, constant greetings, and handshakes. To an observer, however, in the privacy of their own classrooms and offices, divisions and rivalries are quite apparent between the different groups making up the university. 
A tour of the department's building reveals that some students tend to stand in the hallways and stairwells, chatting or playing on their phones, while others wander about or stare at the myriad of schedules and timetables kept under glass. Most students are young, sometimes only nineteen years old during their fourth year, and almost all who regularly attend are female. In the teaching cohort, many students are married or will be married immediately after graduation. The student body features several ethnicities and nationalities, though aside from the hijab, al-amira, or shayla worn by some married or religious female students, it appears to be a homogenous group that rarely acknowledges, much less discusses, divisions among themselves. On several floors, students fill classrooms while waiting for classes or instructors. They appear complacent and talk with one another - or, more commonly, sleep - until they decide it is time to leave. Everyone in the university exudes a notable calm, even during examinations, when events diverge from the schedule, or when things fall apart, almost as if this turbulence is to be expected. To myself as an observer, a certain amount of chaos and confusion was noted throughout classroom observations of university processes and instructional procedures. When participants were asked about this observable chaos, the disconnects in operations and outcomes, many shrugged and smiled or referenced their country or culture. I wondered, is this simply how things are done at this university and perhaps more broadly in post-Soviet higher education?

\section{Data Condensation}

I transcribed and analyzed data from each collection stage using three first cycle coding procedures: descriptive, in-vivo, and values-based as detailed by Miles, Huberman, and Saldaña (2014). I produced verbatim transcripts of each interview from 
the twenty practitioner participants which included current faculty and administrators as well as the twenty student participants. The verbatim transcripts served as emic data as defined by Creswell (2008) as they represented information directly obtained from participants' perspectives. In addition, I conducted classroom observations with seven different instructors, generating data stemming from each of their class sessions. This observational data constituted etic data according to Creswell (2008) as it was obtained from my perspective as an external observer. The verbatim transcripts and observational notes generated the study's qualitative data corpus. I employed three coding procedures to identify and interpret the meaning of the information obtained via interview and observation. Descriptive coding summarizes the content within a passage and generates an inventory of topics (Miles, Huberman, \& Saldaña, 2014). In-vivo coding uses the participants' own words as codes to derive meaning from content (Miles, Huberman, \& Saldaña, 2014). Values-based coding identifies participants' perspectives in terms of importance (values), interpretive social perceptions (beliefs) and the way participants think and feel (attitudes) (Miles, Huberman, \& Saldaña, 2014). Throughout the remainder of this chapter each code is identified by a subheading with its respective coding procedure for additional clarity. In the following sections, I present codes pertaining to each data collection stage and provide a brief description to illustrate their context; full discussion and interpretation is reserved for the final chapter.

\section{First Round of Interviews}

For the first round of interviews, I spoke with administrators and faculty within the ethnographic case. I conducted interviews off campus in a local coffee shop, and the twenty volunteer participants completed the first interview protocol in no more than thirty 
minutes each. While most understood the content of the questions without too much explanation, the quality of responses varied significantly, and some participants struggled to express themselves. For example, when participants were asked to describe their university experience some replied with single word descriptors such as 'good' while others relied heavily on online translators to grasp the question or develop an answer. While I anticipated that participants would use Google translate and other applications, some attempted to translate questions and responses verbatim between English and Russian. In some instances, the rough translations made little sense relative to the questions being asked and I had to attempt to clarify content on several occasions. In addition, a few participants provided answers which in no way related to the study including a passionate political diatribe and questions about getting a visa to the United States. While I was able to guide most participants back to the semi-structured interview content, at least two insisted on providing answers which bared little relevance to student engagement and, from my perspective, appeared to be rehearsed and artificial. Time permitting, I transcribed these twenty interviews directly following the interview and prior to conducting classroom observations. Data from the first transcribed interviews indicated that I needed to adjust my data collection strategies. Specifically, several participant responses were very generalized statements or just a few words in response to open, descriptive lines of questioning.

The data obtained from the first round of interviews indicated that the protocol would need to be interpreted relative to both the linguistic abilities of each participant and their willingness to participate. I elected to conduct extended interviews outside of the protocol with five practitioner participants who possessed exemplary English language 
fluency and proficiency to capture additional rich detail and thick description from the interview data. Where semi-structured prompts from the protocol failed to yield substantial responses from these participants, I checked their understanding of the question and inquired as to whether I could clarify any content. I took very direct approaches in soliciting additional information by having participants expand their responses using follow-up prompts included 'Tell me more about the challenges you have every day in the classroom' or 'What do you mean when you use that phrase and can you provide examples?' or 'Can you tell me about a specific instance when you experienced this?' These changes resulted in interviews which nearly doubled in length but yielded further nuance and clarification of general statements obtained from the first participants.

By striving to transcribe data immediately following the interviews and encouraging participant feedback I was able to gauge the effectiveness of my interview technique and protocols in line with responsive and reflective qualitative fieldwork. A single piece of advice garnered from a senior practitioner in the program had a significant impact on my interview practice and substantially improved the clarity and quality of responses. This practitioner very honestly informed me that any use of colloquialisms would perplex most participants. In reviewing transcripts of prior interviews this became obvious as a researcher, but instances had managed to emerge in dialogue. My confusion as to how seemingly innocuous questions such as, 'How is the program running?' or 'Who runs the university?' was explained at least in part by my failure to use standard English and avoid idiomatic expressions and colloquialisms in dialogue. I observed a marked increase in the quality of interview protocol responses by being mindful of my language and the linguistic barriers which existed between myself and participants and by 
expanding upon interview protocol questions among those participants who were both capable and willing to do so.

In coding the first round of interviews, I followed the procedures detailed in Chapter Three by performing three waves of transcribed content coding: descriptive, followed by in-vivo, and finally values-based. By proceeding in this manner, codes were extracted from the interview transcripts which highlighted different aspects of participant generated content, contributing further strength to the analytical process. Specifically, descriptive coding identified the physical locations from which codes emerged thus contributing additional orientation and perspective within the data. In-vivo coding strengthened the quality and quantity of participant voice by focusing on codes emerging directly from student and practitioner input. Finally, values-based coding sought to develop greater understanding of the values, attitudes, and beliefs underlying higher education experiences and practices within the teaching and learning environment at this institution. As indicated in Figure 8, each coding procedure yielded specific codes stemming from the transcribed data obtained from the first round of interviews, I elaborate on the meaning behind each code below and subdivide the section by procedure type (see Figure 8). 


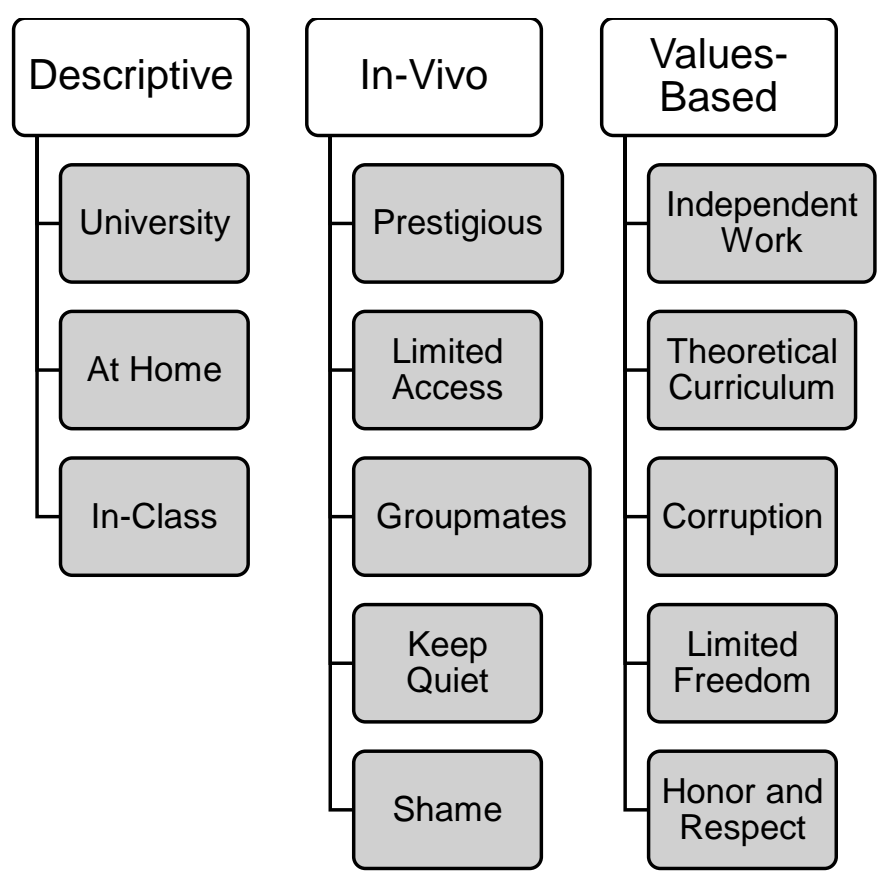

Figure 8. First Cycle Coding Procedures and First Interview Outcomes. This figure depicts the initial first cycle coding procedures and codes resulting from the first round of interviews with practitioners.

Descriptive Coding. Participants from the first round of interviews broached the same topics and key descriptive codes emerged as content indicators. All practitioners identified their undergraduate studies as being in "university," this is significant as in Soviet times studying at a higher education university, vyshee uchebnoe zavedenie or VUZ was reserved for the elite and each major city only had one (DeYoung, 2011). Completing studies in secondary specialized education or technical/vocational education, at the pedagogical institute or teachers' college, or at the military academies would allow an individual some upward mobility but gaining admission to a VUZ guaranteed a career and opportunities for advancement (DeYoung, 2011). Indicators of physical location also proved to be important as participants differentiated between power structures, practices, and behaviors "at home" versus "in-class." Cultural practices within a traditional Kyrgyz 
home as described by participants are patriarchal and delineated by age with elders wielding a tremendous amount of control within their households. While participants described such traditions with reverence, an element of fear also permeated our discussions. Practitioners explained that at home, their time was not necessarily their own as child-rearing and other domestic responsibilities took precedent. This total dependence and lack of freedom was specifically highlighted by female participants who explained that traditional family roles can be limiting, and many face challenges in completing or pursuing education. One participant elaborated, "as a daughter-in-law my time is determined by my mother-in-law, everything depends on what the family needs and what she says." Specifically, family dynamics and traditions created problems for some participants when professors assigned homework or independent assignments as domestic responsibilities often took precedent. A participant remarked, "teachers accept no excuses. I made the choice to get married... have a family. So, if my kids are sick and I must be absent, it is my responsibility."

In describing their undergraduate experiences, participants focused almost exclusively on what happened in class. Several remarked about their roles as students, explaining that in class, "we noted every word, what our professors said were the facts and we had to know them." Also, "we were afraid, the experts teaching us knew everything and what did we know?" Residence-life is essentially unknown at the research site as campus housing is not provided by the university; students elect to room in off campus apartments or with relatives and often must return to their home village during weekends, on breaks, or as needed. Lines of questioning focusing on what happened outside of class at university were glazed over by many participants as they 
implied that the bulk of their time inside the university as undergraduates was spent attending courses and lectures. Participants described campus life experiences beyond instruction as typically taking place off campus and remaining outside of the purview and general control of the university itself. The divisions between university, at home, and in class highlight the different power structures and dynamics in place across locations impacting participants at the research site. A current student stated, "when we leave university for the day or week, that's it, we are with our families and have to do as told."

In-Vivo Coding. Practitioners spoke of their undergraduate experiences using specific vocabulary and phrases which I identified as recurrent in-vivo codes. These codes served to empower participant input by including content obtained directly from their responses to include their respective voices in this study. Participants expressed a certain pride in attaining a university degree and the actual university from which it is earned was also indicated as being of importance thus the code "prestigious" emerged when discussing the quality of higher education institutions. Participants described the challenges they faced in completing their studies under the overarching code "limited access." Particularly during Soviet times, simply locating the textbooks needed to complete assignments was extremely difficult as the university restricted library access and required materials and English texts were both expensive and in very short supply. Further complicating the issue was the lack of technology to scan or duplicate documents. Senior practitioners described spending hours copying text after text and many proudly displayed shelves of their hand-copied notebooks. One stated, "just getting the information or a single English book was so difficult then. It forced us to work hard and work together to do our assignments." 
Access was a persistent theme throughout university descriptions as there really wasn't any space within the university for students to congregate and meet except for when they were in a classroom or in hallways waiting for a class to begin. Other in-vivo codes included "groupmates" to explain an educational practice in place since Soviet times, where students are placed in small cohorts that progress together through each year of university. Some practitioners established lifelong friendships with some of their groupmates and described supportive learning communities between one another while others said that their responsibilities at home prohibited their full participation in the group outside of class. A student from a large family stated that, "At home I have two very young sisters and I help my mother. She teaches and works so much. If they are sick, I must stay and take care.”

In classrooms and within student-faculty interactions participants consistently used the in-vivo code "keep quiet" to describe the cultural practice of not criticizing or creating trouble for administrators or instructors. While an outside observer could view keeping quiet as being an indicator of being disengaged or unempowered, practitioners explained the cultural practice further. Some simply attributed keeping quiet to shyness, with hesitancy among students to voice their opinions as their families being very conservative, religious, or traditional. Others explained this as a fear of being singled out or even scolded for interrupting or questioning the instructor and many referenced that throughout primary and secondary education it was important to remain part of the group and not bring attention to oneself. A senior instructor noted, "As students we were afraid of being pointed out in class or not having the right answers. If we said nothing, it was better than being wrong." 
"Shame" emerged as another in-vivo code that practitioners used to describe feelings when speaking Kyrgyz over Russian or using Southern dialect and expressions, and even to justify why students sometimes accept nonsensical advice and incorrect information from superiors without question. Participants elaborated that to be shamed publicly must be avoided as it can move beyond self-reproach to negatively impact parents or families in multiple spheres of life. A junior instructor remarked, "my mistakes are not my own. To damage my family would be terrible." Similarly, a student stated that, "even in English, if a teacher just says 'shame' about something I do or forget, it can ruin my day." Participant interviews revealed that the fears participants expressed concerning shame ties into the cultural practices which act in unison to restrict freedom and independence among youth and learners within the program.

Values-Based Coding. The final coding procedure sought to identify what participants valued as important, their thought patterns concerning themselves, people, things, and ideas as evinced by attitudes, and their beliefs which encompassed both values and attitudes. In terms of values, many participants cited "independent work" as being key to student learning, academic achievement, and ultimately success. Practitioners readily admitted that a university degree could and still can be obtained at the research site with limited student effort for the right price but at the expense of learning and skills. One faculty member stated, "if they want the skills with the diploma it is up to them, they can get the diploma...even doing nothing." Faculty give assignments for students to complete at home, but the final grade earned in every course is based entirely on final examination results, again a predominant Soviet practice which has changed little over the ensuing decades. An administrator recalled, "As a student, 
final marks were assigned and that was it. Even with my doctorate, professors could make it very difficult or easy, it was clear."

Study participants expressed frustration with educational practices and injustices that have persisted despite changing times, an attitude code which emerged as "corruption." Participants used corruption to describe different practices from final examinations with subjective pass/failure standards, professional selection for educational training or fellowships, and the allocation of instructional hours which ties directly to compensation. Several participants simply stated, "it is our culture," or "people expect something for everything," and shrugged, effectively accepting it as practices. A degree of fear was also evident in participant responses in describing how they found it necessary to continue to employ such practices to maintain and hopefully advance their status in the university. A junior faculty member stated, "It is tribalism, I think. Seniors have their groups and favorites and they are all friends, and some are family, so they help each other all the time. But who could I complain? What can I do?" "Theoretical" emerged as a recurrent critique of the university curriculum, specifically that it lacked ties to practice — even the teaching programs were described as focusing exclusively on pedagogical and linguistic theories. A student interviewed on this matter pointedly responded, "This is a teaching program without methodology. They should just start over. New program. New teachers."

Nearly every first interview contained belief codes like "limited freedom" and "honor and respect." Practitioners explained that students needed limited freedom for optimal order and learning and acknowledged their relative independence in determining how limited freedom for their students should be. This belief was described at once a 
Soviet vestige and a modern cultural norm at the research site as the student experience remains regimented with little opportunity for debate. Honor and respect emerged as two critical cultural components that appeared to dictate everything from acceptable classroom behavior to student-faculty interactions. Practitioners expanded on the critical nature of these beliefs, stating that student insolence would not be tolerated by faculty or administrators, or even other students. An administrator stated, "We have to control students, otherwise there will be chaos. Strong instructors do this very well." The consequences of being labeled as disrespectful or dishonorable are so significant that even broaching the topic with some participants was unconscionable with some speaking in absolutes, "That would never happen," or "We would never do that." The valuesbased codes served to reinforce the emerging ethnographic themes of fear, independence, and freedom by highlighting cultural practices within the university which supported their commonality.

\section{Classroom Observations}

Although every practitioner agreed to let me observe their classes, this was logistically impossible because the university had recently changed to one-hundredminute classes with a single ten-minute break. Classroom sizes, instructional methods, attendance, and content all varied considerably. For example, senior practitioners were typically allocated slightly larger classrooms while junior practitioners were left to find an open space or given whatever room was available. Observed instructional methods ranged from lectures to workshops to small group projects and presentations depending on the instructor. The quality of instructional content was observed to vary from lectures lifted from the internet to canned lessons from English preparation texts to archaic 
content from obsolete texts. Students and instructors knew in advance that I would be observing their classes; however, in two of the smaller classrooms, my presence essentially mandated my involvement in the class. While foreign teachers are present periodically throughout the university, they typically offer lectures or speeches and rarely interact with students directly. By sitting with students, some felt comfortable speaking to me and asking questions, and notably the same questions predominated 'How old are you?' 'Where are you from?' and 'Are you married?' As a researcher, my intent had been to remain an independent observer of classroom behaviors, however my interactions with students and practitioners in most cases didn't appear to be distracting or to impact the class observations significantly. I interpreted my unintentional classroom interactions as being representative of two target student behaviors: engaged student interaction and engaged interaction with an instructor. The adapted classroom observation instrument (see Appendix F) served its purpose well, and I continued documenting each class even while tangentially participating. Of note, there were constant interruptions to the learning environment: random people opening and closing the door, fellow practitioners and students entering briefly to ask questions, and students interrupting class to ask permission to leave or attend. Students and instructors would acknowledge these disruptions without concern or simply shrug and go about their classwork. I witnessed many similar interruptions during my fellowship experience, but I found them particularly distracting and disruptive during my classroom observations even though instructors and students tolerated them without complaint. Interruptions that I found unacceptable during my fellowship appeared as common cultural practices for this program when being viewed and interpreted as a qualitative researcher. 
My review of the classroom observation instruments yielded several codes via invivo analysis of the seven completed observation instruments. As indicated in Figure 9, I traced observational evidence from my field notes which supported the codes and provided justification for their inclusion in the development of the ethnographic themes.

\begin{tabular}{|l|}
\hline \multicolumn{1}{|c|}{ Passive } \\
\hline - Copying \\
board text \\
- Extensive \\
lecturing \\
- Restricted \\
student- \\
faculty \\
interaction \\
- Memorization \\
and recitation \\
- Indifference to \\
punctuality \\
\hline
\end{tabular}

\begin{tabular}{|l|}
\hline \multicolumn{1}{|c|}{ Disengaged } \\
\hline - Cellphone \\
usage with \\
headphones \\
- Off-topic \\
discussions in \\
non-target \\
languages \\
- Sleeping and \\
unresponsive \\
- Limited \\
project/task \\
completion \\
\hline
\end{tabular}

\begin{tabular}{|l|}
\hline \multicolumn{1}{|c|}{ Archaic } \\
\hline - 1970s \\
philology \\
textbook \\
- Phonology, \\
lexicography, \\
morphology, \\
and syntax \\
- Translation \\
approach \\
- Outdated and \\
formalized \\
English \\
\hline
\end{tabular}

\begin{tabular}{|l|}
\hline \multicolumn{1}{|c|}{ Deference } \\
\hline - Asking \\
permission to \\
enter/leave \\
- Arranging and \\
cleaning \\
classrooms \\
- Constant \\
apologies \\
- Expressions \\
of Gratitude \\
- No pushback \\
to errors \\
\hline
\end{tabular}

Figure 9. First Cycle In-Vivo Classroom Observation Codes. This figure depicts the in-vivo codes obtained through the seven class observations conducted and the corresponding sources of evidence from the adapted classroom observation instrument (see Appendix F).

Students and instructors were notably "passive." Some classrooms were markedly quiet except for interruptions and noise from the hallways; the instructors spoke little, and students spoke even less to the point that I sometimes felt that I was observing a room with nothing happening at all. When literally nothing was happening aside from idle chat and interruptions, I wondered why students tolerated this and why they even bothered to attend. I described students themselves as "disengaged" in most instances, with frequent 
behaviors that I considered disrespectful—listening to headphones during class, sleeping, or putting their heads down on their desks — but these were tolerated and even acknowledged by instructors. I typified the content of classes as "archaic," lacking relevance and being highly theoretical in nature. The department itself and most of the degrees awarded reference philology, a classical discipline loosely related to the study of language structure, development, and languages rather distant from language learning or teaching. I questioned the functional value in memorizing the phonetic chart and reading through a phonological science text from a 1970s Russian textbook, though I was impressed that some students could recite every component. When a subject matter piqued a student's interest, they were notably interactive, but I observed this to occur in small student group discussions only. Students interacted with faculty in a traditional manner, raising their hands to answer questions and accepting a curt 'yes' or 'no' in response. Some students took copious notes throughout lessons, attempting to transcribe every word that instructors wrote on the blackboard. The one-hundred-minute class sessions felt extremely long as an observer and some students became visibly restless toward the end of classes. One instructor particularly tested my attention and patience as an observer by lecturing from a prepared speech primarily in Russian in a low monotone for the entire English literature session. At the end of that class - and every observation, in fact — students received homework assignments with limited explanation, waited for dismissal, packed their things, and individually thanked the instructor for the lesson in what I viewed as a final indication of student "deference."

\section{Second Round of Interviews}


I interviewed students after the classroom observations at the end of classes for the day and prior to the second round of interviews with practitioners. While I followed the interview protocols for practitioners, I used a less structured format for student interviews and sometimes found it necessary to interview multiple participants at once. This was an intercultural consideration as traditional gender roles would make an interview between a male researcher and an individual female student even in a public space highly questionable. Students were more likely to participate in small groups even when the interviews were predominantly one-on-one. Again, because of language difficulties, I interviewed three students more thoroughly than others about their higher education experiences. Although I interviewed twenty students and they were candid with criticisms of their university program, I conducted more in-depth interviews with the three particularly informative participants. In conducting the follow-up interviews with practitioners and asking students about student engagement practices, I discovered that while I had provided the NSSE benchmarks in advance via WhatsApp and hardcopy, few participants appeared to have reviewed them. I therefore explained each benchmark and its components to each participant, so my input was greater than planned and the second round of interviews ran longer than intended. By explaining each benchmark, I potentially influenced participant responses and noticed some repetition of my explanations in responses. In addition, I ended up conducting fewer second round interviews than first round interviews because of constantly changing schedules, noshows, and interruptions that turned to cancellations. In total, four practitioners could not be located to conduct their second-round interviews, however the five selected for extended interviews all participated and contributed greater depth to the transcribed 
interview content. Figure 10 reveals the first cycle second interview codes derived from the transcribed interview content.

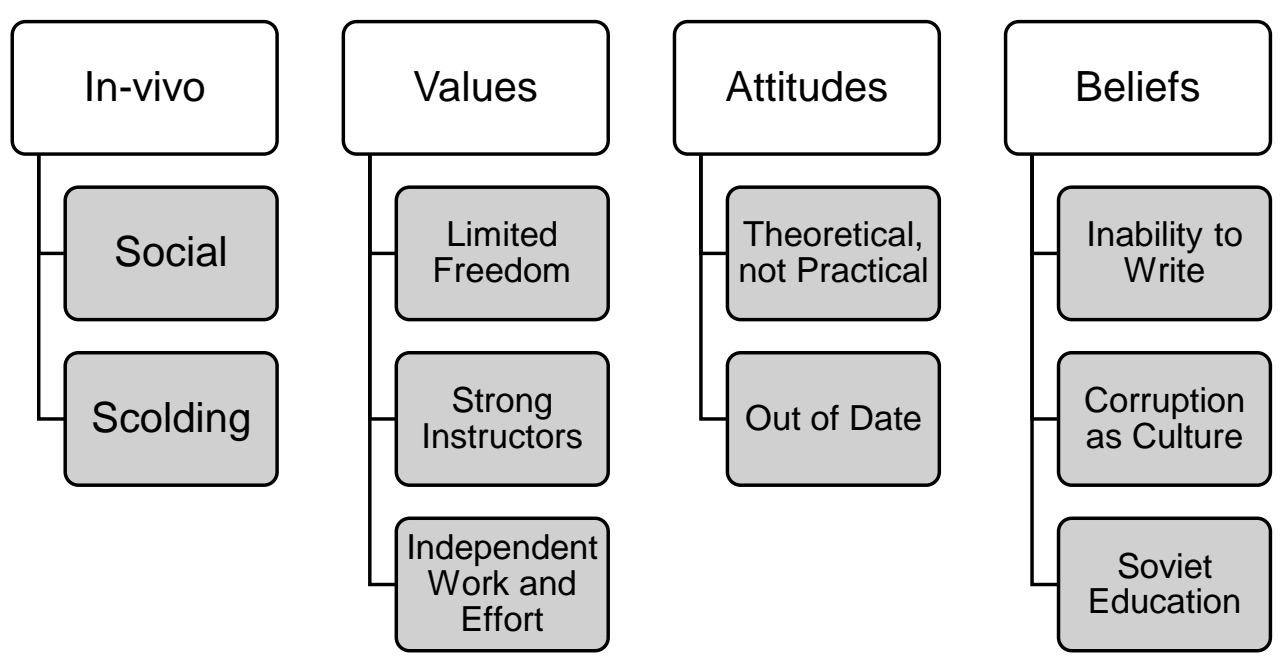

Figure 10. First Cycle In-Vivo and Values-Based Second Interview Codes This figure depicts the coding procedures used to analyze the second interviews with student and practitioner participants and presents the resulting codes and introduces their corresponding evidence sources.

In-Vivo. Once I reassured students about confidentiality, they were surprisingly candid, explaining in-vivo that their university experience was "social" but did not meet their expectations. Several alluded to major problems in the program and described it as a "waste of time and money." Many students stated that they were primarily there to interact with people their age at the urging of their parents. One remarked, "I don't want to be a teacher, but my father said it would be good. So that is why I come." Practitioners, many of whom are alumni of the program, also stated that the social component of university, working together with groupmates and building connections with people from other families were essential parts of the experience. A junior 
instructor remarked about a colleague, "He knew he didn’t want to teach. He played guitar all the time and did very little studying. He used his connections here to start a wedding orchestra business. Now he makes more money than all of us."

The importance of family and traditional values within this culture were significantly represented participants' perceptions and beliefs of their higher education experiences. Elders and superiors often verbally shamed students and colleagues, a practice identified by the in-vivo code "scolding." A senior instructor recalled from her days as a student, "I had a Soviet phonology teacher who was so serious, she never smiled. She scared us all so much. If we got the answer wrong, even partly wrong, she would shout at us in front of everyone. I would pick a spot on the floor and just stare until it was over." The avoidance of shame by students and junior practitioners was evident in their descriptions of being scolded for incorrectly answering questions, much less asking questions. Participants described this practice as explaining some of the tolerance for instances of corruption including instructors suggesting bribes guised as gifts in exchange for passing examination scores.

Values. Practitioners explained that many of the benchmarked practices were already in use implicitly but terminology such as student engagement and stakeholder outcomes were unknown until they arose, some as part of an accreditation process. Students took an adversarial stance toward the benchmarks, stating that among themselves such practices took place, but practitioners dictated the classroom environment. An administrator conceded that, "We don't use benchmarks. What teachers do in their classes is up to them. I provide guidance but am not a supervisor." Again, both students and practitioners referenced "limited freedom" as a value placed on 
instructor oversight or the lack thereof. Restated, participants stated that they perceived value in limiting student freedom with justifications including control and power over the university process and instructional practices. Administrators echoed some of these concerns for the predominance of "strong instructors" as a value code describing how the strength of an instructor echoed their dominance within classrooms over students. Interestingly, the same senior instructor who described an unsmiling and ruthless Soviet instructor later described her as "My favorite teacher who I never will forget. I learned so much and respect her work so much." Administrators indicated some indifference in stating that teachers sometimes made mysterious use of their time, complaining of being overworked but also requesting more instructional hours. On the other hand, instructors stated that it was up to students to get something out of their education, and some stated that it was a pity when students chose to waste their potential on an effortless education resulting in a useless diploma. All participants deemed "independent work and effort" as being a value that is essential to success. A young instructor stated that "It matters who you are, it matters what you do. If you do nothing, nothing good will come." Students and practitioners alike placed responsibility on learners to get something out of their education and improve their outcomes. The reality that comparatively few students are empowered to do so was acknowledged by several participants, with practitioners conceding that some who attend university only seek to obtain a degree and little else. One student participant admitted, "I attend university because of my father. He wanted me to go because he couldn't, so I do. It is what he wants."

Attitudes. In elaborating on their unmet expectations for university many students and practitioners described their classes as "theoretical, not practical," and 
expressed concerns about their ability to teach or work as interpreters/translators in the future. Participants described this attitude code as an expression of frustration with the disconnects between higher education expectations and realities. A student participant stated "I do not know what I am doing here. I want to have a career, but I think it will not come from a diploma from here." Aside from a handful of young instructors and specialists, some practitioners and their practices were perceived as being "out of date"; they repeated their lessons year after year or filled classes with irrelevant discussions. While senior colleagues and elder professors are highly revered throughout the university, participants admitted that their classroom practices and curricula were tolerated out of respect even though they were perceived as being less than optimal for learning or satisfaction.

Beliefs. All participants, but especially those in translation, identified an "inability to write" as a huge detriment. This belief code can also be indicative of the failures of the program to meet participant expectations or obtain the skills necessary to succeed. A student lamented, "I do not know how to teach. I cannot write well. This makes me worry about what I will do.” Toleration of fully plagiarized papers was attributed to a unilateral writing skill deficit by participants. One administrator conceded, "Writing is a weakness in the program. Many teachers have not published or completed research because of writing. It is a big problem throughout Kyrgyz education." Participants also described "corruption as culture" a belief code that summarizes the perception of corruptive practices as a component of nearly every sphere of life in this region. While corruption is overtly discouraged, the practical reality is that it forms a cultural practice reflected throughout the educational process in this region. A senior 
instructor admitted, "This is how things get done and it was how things got done in Soviet times. The people you know and the people you help in turn help you. Corruption is everywhere, but we manage it." Some practitioners evaded questions pertaining to the benchmarks and instead discussed "Soviet education" a belief code explaining the generally positive attitudes and values pertaining to educational development and practices throughout that era. Participants conceded that the very existence of the university was due to Soviet planning and its ongoing administration and classroom instruction paid homage to Soviet educational practices. A newly qualified instructor stressed, "Some elders would go back to Soviet times, even in the university. They say that everything was better. My memories are different, and it doesn't exist anyway." This concession is reflective of the realities of life and education at the research site as the institution itself as well as its practitioners and students are at once defined by a Soviet historical basis yet constrained by diminished resources and forced to operate within a modern global context.

\section{Ethnographic Themes}

I elicited predominant ethnographic themes from secondary pattern code analysis of the condensed data. The intent of the narrative analysis, checklist matrix, and participant vignettes were to display the condensed data in context before being used to extract and support the elicited ethnographic themes. Chapter Five provides detailed analysis and interpretation of the ethnographic themes themselves as part of conclusion development and verification. In the sections which follow, I offer a narrative analysis to interpret how things work within the ethnographic case and to highlight essential thematic features (Creswell, 2008). This narrative analysis represents negotiation data as 
defined by Creswell (2008) as I composed it as an external observer then refined the narrative with key participants to ensure that it reflected reality. A checklist matrix examines elements of the NSSE benchmarks in further detail, incorporating both emic and etic data. Finally, I selected participant vignettes that portray defining moments from the entire collection of interviews and epitomize the emic data obtained by interviewing individuals within the culture-sharing group under analysis. As indicated by Figure 11, I analyzed the codes elicited through data condensation in display via narrative, identification of the presence or absence of benchmarks in a checklist matrix, and by drawing from participant vignettes. These analytical processes supported one another and contributed additional trustworthiness to the study's findings and their interpretation as detailed in the figure below.
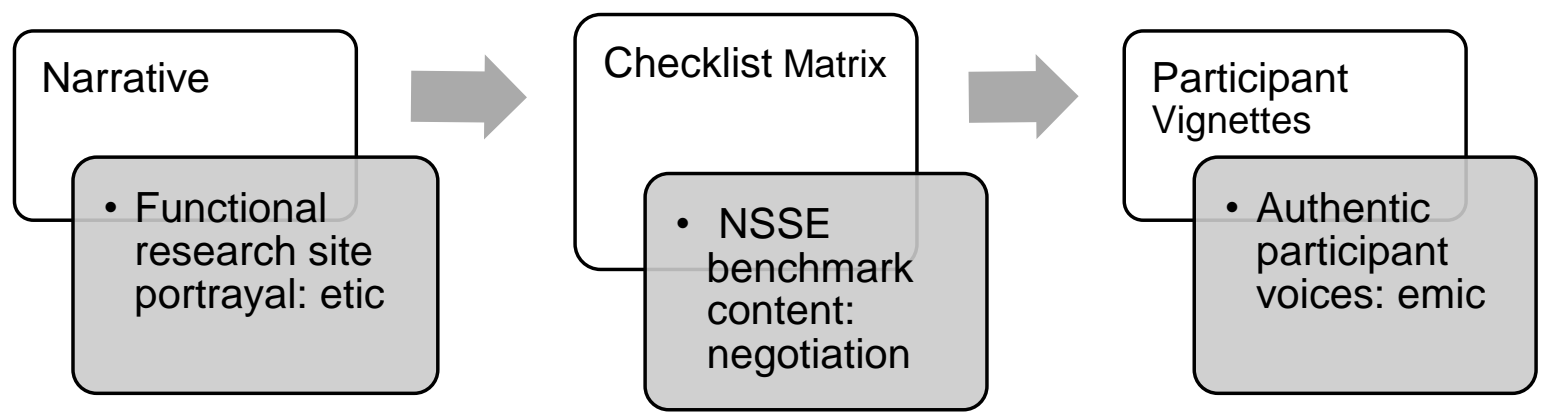

Figure 11. Secondary Code Analysis for Ethnographic Themes. This figure depicts the coding procedures used to further analyze the classroom observation and student and practitioner participant interview data.

Narrative Analysis. I took the opportunity to display the elicited codes in their context to generate further understanding via narrative analysis. In telling the story of the students and practitioners comprising the program under study, I place the codes yielded from data collection in their situated context and generate additional meaning. In using this method, I reconstructed the practical realities of education within this program using 
the codes as an empirical basis for telling the story. The story of education in this program itself is an interpretation of its associated teaching and learning environment. The content for the narrative developed through an amalgamation of the study data obtained from study participants in interview and via classroom observations. In addition, this analysis reinforced data trustworthiness through triangulation between observational and interview data sources, and member checks of the conclusions between three key participants: a student, an administrator, and a faculty member.

Practitioners concede that students primarily enter this program to obtain a diploma, often at the order of their parents. Students admit and demonstrate that they are not fully aware of the diploma they are earning or the requirements for earning it, much less what they will do with it after they graduate. Predominant cultural norms mandate absolute deference to the orders of parents and others, so students set aside personal ambitions and dreams for fear of violating what their elders consider best. Although university admission technically depends on national scholarship testing, students can override that requirement by completing a preparatory year or making a financial contribution to the department.

After gaining admission to the program as a 'contract' student or receiving a 'scholarship' spot, students begin a class schedule largely dictated by the administration, with little room for personal choice. Class attendance is mandatory as the diploma mentions 'seat hours,' though in practice, students forge the sign-in easily or pay to have their documented hours changed at any time prior to commencement. Although the Ministry of Education and Science for the Kyrgyz Republic sets the official curriculum, the university considers it less of a mandate and more of a suggestion. Instructors must 
submit their lesson plans, content, and examinations at the beginning of the term, so they often pass same content and curriculum down and around. Administrators claim to have recently aligned the university with the Bologna process by switching to a four-year bachelor's degree format, but they cut course content drastically in the process.

Instructors have the freedom to deliver their classes with limited administrative oversight. However, this freedom is restricted by time constraints, inadequate facilities, dysfunctional technology, and fluctuating student attendance. Many instructors follow a third-party English textbook word-for-word from the teachers' edition or deliver lectures verbatim from the Internet. Some students take notes in tiny spiral books and pay attention or appear to the whole time, while others play on their phones, sleep, or ask permission to leave. Instructors assign independent homework but do not penalize students for non-completion. In fact, most instructors spend considerable time covering the assignment during the subsequent lesson.

Few students complete the independent work of their own admission, but they are the ones who, according to administrators and faculty, eventually find exchange programs and academic opportunities to leave and better themselves. In class, students are observed to rarely ask questions, express interest, or state opinions. Communication in the classroom environments is consistently observed to be unilateral with the professor as an expert responsible for lecturing and students responsible for taking notes and completing assignments. Some faculty admit to and visibly demonstrate limited interest in teaching English or English itself as the curriculum remains centered around linguistic theories and content that is nearly fifty years old and in Russian, which not all students understand. Students fear being shamed for asking questions or questioning content; for 
their entire lives, they have encountered only a transaction fact-based approach to education. Students have restricted freedom within the classroom as behavior in schools and classrooms is socialized from a young age to emphasize conformity and deference. Student freedom is also curtailed within the greater university as class schedules and degree programs are set by administrators with little flexibility or choice for individual students. In addition, respect for elder faculty and administrators exists on an absolute level and behavioral problems and insubordination are essentially nonexistent while frustration admittedly seethes under the surface. The university's classroom environments and practices are observed simply to not feature critical analysis or thinking. Practitioners state that students excel at memorization and can complete projects if given what educational theorists refer to as proper scaffolding and sheltered instruction. However, practitioners face financial constraints which limit their efforts and time constraints that effectively prevent them from offering adequate support. Groupmates, cohorts of six to eight students, proceed through university together. When the group works, it becomes a support network independent of the campus environment.

Volunteer teachers and some students use the administration for unpaid tasks such as accreditation preparation, conference coordination, or even to fill a room for a meeting with a government official — thus, classes can and are cancelled with no notice. Students and practitioners acknowledge that some receive a diploma without earning it. This practice has existed since the founding of the university during Soviet times, although admission, graduation, and certainly gainful employment and job security are no longer absolutes. The teaching credential officially depends upon passing examinations, attendance or 'seat hours,' completing a practicum, and composing a final written 
diploma paper. However, the university will overlook all these requirements in return for a few thousand Kyrgyz som. In a cruel twist of financial dependence, students on scholarship must teach for three years at a public school prior to receiving their diploma. Official university policy strictly prohibits bribery and corruption, but some instructors depend on bribe money to supplement their poorly compensated teaching hours. In addition, instructors fear negative student reviews, which in theory could jeopardize their course load and salary. Administrators, including chairs, deans, and rectors, are in a state of flux with constant changes, promotions, demotions, and replacements. From the perspectives of students and practitioners, administrators operate independently of their own university, and many hold their positions for the sake of earning power and prestige rather than a living.

Fear, independence, and freedom pervade this teaching and learning environment and explain how status quo practices, procedures, and outcomes remain largely constant.

Checklist Matrix. The matrix in Table 2 summarizes findings on perceptions of the NSSE benchmarks as noted during interviews and observations within the teaching and learning environment of the ethnographic case. Applying both emic and etic data obtained from internal and external perspectives respectively yielded evidence supporting the presence or absence of the benchmarked practices. This matrix serves to support data trustworthiness by accounting for negative or discrepant cases where practices were not observed or described by participants. As displayed in this manner, the benchmarks are used as a lens for understanding the educational conditions present within the program and the nature of the teaching and learning environment at the research site. 
Table 2.

Reflections and Perceptions of Educational Conditions and Practices Supporting Student

Engagement among Practitioner and Student Participants

\begin{tabular}{|c|c|c|}
\hline NSSE Benchmarks & Practitioner Perspective & Student Experience \\
\hline $\begin{array}{l}\text { Level of Academic } \\
\text { Challenge }\end{array}$ & $\begin{array}{l}\text { 1. Exams were difficult yet } \\
\text { practical with translation } \\
\text { and interpretation tasks } \\
\text { 2. Few resources and very } \\
\text { few textbooks, student } \\
\text { cooperation and laborious } \\
\text { hand-copying to prepare } \\
\text { 3. Independent academic } \\
\text { work to develop skills, } \\
\text { perfunctory final paper } \\
\text { 4. Critical analysis was } \\
\text { actively discouraged, } \\
\text { theoretical curriculum } \\
\text { 5. Course difficulty was } \\
\text { tied to student effort and } \\
\text { faculty expertise }\end{array}$ & $\begin{array}{l}\text { 1. Multiple choice exams } \\
\text { are prone to cheating and } \\
\text { instructor influence } \\
\text { 2. Limited access to } \\
\text { resources on campus, but } \\
\text { increased technology, little } \\
\text { at-home preparation } \\
\text { 3. Optional homework and } \\
\text { projects, perfunctory } \\
\text { course and diploma papers } \\
\text { 4. Specific critical analysis } \\
\text { tasks, limited application, } \\
\text { judgment or synthesis } \\
\text { 5. Course difficulty is tied } \\
\text { to faculty abilities and } \\
\text { interests, less student effort }\end{array}$ \\
\hline $\begin{array}{l}\text { Active and Collaborative } \\
\text { Learning }\end{array}$ & $\begin{array}{l}\text { 1. Traditional Soviet } \\
\text { instructional methods, } \\
\text { lectures and recitation } \\
\text { 2. Student questions and } \\
\text { in-class discussions } \\
\text { restricted to curriculum } \\
\text { 3. Student cohort } \\
\text { collaboration was essential } \\
\text { at university and at-home }\end{array}$ & $\begin{array}{l}\text { 1. Traditional and modern } \\
\text { instructors, lectures and } \\
\text { small-group seminars } \\
\text { 2. Questions and } \\
\text { discussions are determined } \\
\text { by the instructor } \\
\text { 3. In-class cohorts are } \\
\text { collaborative, but students } \\
\text { work independently }\end{array}$ \\
\hline $\begin{array}{l}\text { Student-Faculty } \\
\text { Interaction }\end{array}$ & $\begin{array}{l}\text { 1. No feedback on } \\
\text { academic performance } \\
\text { until course pass/failure } \\
\text { 2. Interaction limited to in- } \\
\text { class and during exams, } \\
\text { faculty offices restricted } \\
\text { 3. Bribery and corruption } \\
\text { acknowledged as accepted } \\
\text { university practice }\end{array}$ & $\begin{array}{l}\text { 1. Limited student } \\
\text { feedback until exams, } \\
\text { instructor dependent } \\
\text { 2. Faculty interaction } \\
\text { limited to the university, } \\
\text { offices are now classrooms } \\
\text { 3. Bribery and corruption } \\
\text { shrouded as university } \\
\text { policy and procedure }\end{array}$ \\
\hline $\begin{array}{l}\text { Supportive Campus } \\
\text { Environment }\end{array}$ & $\begin{array}{l}\text { 1. No student services or } \\
\text { support, independent } \\
\text { choices and responsibilities }\end{array}$ & $\begin{array}{l}\text { 1. Alleged student services } \\
\text { and support, financially } \\
\text { dependent choices }\end{array}$ \\
\hline
\end{tabular}




\begin{tabular}{|c|c|c|}
\hline & $\begin{array}{l}\text { 2. Administration and } \\
\text { faculty inaccessible or } \\
\text { unknown to students } \\
\text { 3. University was formally } \\
\text { academic with few planned } \\
\text { social events or activities }\end{array}$ & $\begin{array}{l}\text { 2. Faculty and } \\
\text { administration determine } \\
\text { their own accessibility } \\
\text { 3. The social aspect of } \\
\text { university is sizable, } \\
\text { student cohorts organize }\end{array}$ \\
\hline $\begin{array}{l}\text { Enriching Educational } \\
\text { Experiences }\end{array}$ & $\begin{array}{l}\text { 1. Soviet emphasis on unity } \\
\text { over diversity across } \\
\text { race/ethnicity/values } \\
\text { 2. Suppression of } \\
\text { alternative political and } \\
\text { religious discussions } \\
\text { 3. Active practicum, } \\
\text { directed international } \\
\text { study, local opportunity }\end{array}$ & $\begin{array}{l}\text { 1. Diversity exists but is } \\
\text { unexamined and undefined, } \\
\text { values remain stable } \\
\text { 2. Hesitance to discuss } \\
\text { race/ethnicity/nationality } \\
\text { and some religious tension } \\
\text { 3. Passive practicum, open } \\
\text { study and travel abroad, } \\
\text { critically low domestic jobs }\end{array}$ \\
\hline
\end{tabular}

Note. Adapted from Billups (2014) and Miles, Huberman and Saldaña (2014).

Participant Vignettes. I selected three participant vignettes among the many

transcripts of students, faculty, and administrators who volunteered to be interviewed for

this study. These vignettes are a source of emic data as they are unedited statements from participants and lend voice to practitioners and students alike. Their display and interpretation are central to ethnographic qualitative inquiry as they contribute thick description to fully illustrate identified codes and triangulation by supplying multiple sources of data as evidence. When I asked about the obstacles students faced outside of the classroom, a current faculty member described her own challenges as a young mother and student:

It was my responsibility I think, mostly, they accommodated me when I was giving birth, but I had trouble with some teachers, after my first son I had to miss one month of classes. I know the problem was my absence, but it was a reasonable absence, but anyway. Then I understood that I couldn't use my family as an excuse for interrupting my studying. I tried not to miss class. I had some support from my chairperson, who I wrote my diploma paper for, when I graduated I was an example for women who got married during university, they made me a sort of example. It absolutely depends on your family and the support that you have; our culture is different. A girl, when she gets married, the family is first with the household and everything, the same might have happened with me when I tried to combine. But I was afraid in the future that my sons might have 
an uneducated mom, I wanted to be an educated mom. Women who sit at home with their children, they do not have a voice or rights I think, they become dependent in financial parts as well, that is why I worked hard.

This participant's accomplishment is notable in that many students who marry prior to graduation do not complete their studies. I spoke with other women who validated this participant's reflection on the traditional role of women in this culture.

As an administrator in the university, the participant of the next vignette touches the benchmarks' potential within a program in a post-Soviet context:

I was lucky enough to travel and study in the U.S. in an exchange program and I was amazed by the fact that everything was online. I know that our students want to obtain such professions as in the U.S., but here some do not want the knowledge, they only want the diploma. This is a leftover, a major drawback of the Soviet system. It used to be you were guaranteed a secure life, a career, a home, if you had a diploma. Life is not this way anymore. The benchmarks will not work here at the moment for many reasons. I know in the U.S. professors have office hours where they do research and meet with students. We have office hours here, but they don't work with students or developing themselves as instructors. I see some who are interested, but here teachers are only paid for teaching. Sometimes I think the enrollment fee may need to be raised to attract better students. I think that would improve the university the most.

From my perspective, students often spoke more pointedly and candidly about their experiences in interview compared to administrators or instructors. In this final vignette, a student who had the opportunity to study in an American high school touches on effective practices and the benchmarks:

Our major is lexicology and interpretation and translation, it would be good if we had more practice. It's more theoretical than practical. We had almost no opportunity to translate something real outside of the university, that's terrible, I know. When I think about the best teachers, I think that if people are passionate or not passionate about something it shows. I want teachers to inspire myself and others because if you are inspired you inspire others, showing interest in what you are doing. Be honest and straightforward with students, it's simple but essential. They play a very important role. About the benchmarks...I think volunteer work; our people are not used to working for free. It's an individual thing I suppose. American students are active, our students are not, they prefer to stay at home and do nothing. There are so many technical problems here that presentations don't 
work. There are no projects, I haven't found any projects here. Work with classmates outside of class and outside of the university, it doesn't work here, home is very separate. What happens in class, stays in class, I think most students just keep everything at university, we don't have such discussions. Overall, more no than yes.

These selected vignettes highlight the complexity of the perceptions and experiences of individuals with varying roles within this teaching and learning environment. They also highlight impediments to educational change in a context that is rooted heavily in the past and is unprepared and perhaps in some ways unwilling to adapt to the future.

\section{Ethnographic Themes}

Fear. Nearly every participant description of higher education included a component of fear, with self-evident effects on teaching and learning. Practitioners and students often referenced their experiences at home as reflections of Kyrgyz cultural traditions and practices. Participants described the nature by which traditional homes operate and revealed that elders yield significant control over every aspect of every member's life within the extended family. When participants were asked why they were pursuing a teaching degree or how they decided to become faculty, responses such as: "I wanted to be a doctor, but my father said I should do this," or "This is what my parents wanted me to do," were not uncommon. Honor and respect are described as sacred values within households and participants described this as extending into the university. When asked about consequences for defiance within a family, participants had difficulty voicing the severity of actions that would accompany such a violation with one participant stating, "No one would do that." The respect harbored for parents, superiors, 
and elders is in many ways absolute, and questioning their knowledge or decisions could make life very difficult for an individual.

Deference emerged in the university relative to senior faculty, administrators, and even unofficial student group leaders. As an external observer, it was of interest to note that gender was less of a factor in inculcating deference in students as was age with the most experienced instructors commanding the most respect. Those senior instructors and administrators were described as yielding significant power, even if only through scolding junior practitioners and students. One instructor remarked, "I loved my teachers even in primary and secondary school. They were very strict, and I remember being frightened at red ink on papers and the look on their faces if my answer was wrong." Participants described a fundamental fear of receiving shame from peers or elders, and many regarded being publicly reprimanded as the ultimate in shame. Senior practitioners described strong instructors from Soviet times as non-smiling subject matter experts who were there to give lectures, ask questions, and assign grades. Participants explained that to question content, give an alternative answer, or highlight a potential error could result in being scolded and shamed in front of the class. A senior instructor recalled, "Having to stand in front and be shouted at was the worst. Stronger instructors expected perfection. I never wanted to talk in class as I could make a mistake." The fear of being publicly reprimanded was described by many participants relative to their academic failures, implying that it may have negatively affected their academic performance and overall motivation. Behavioral problems are reported by practitioners to be few and far between as students opt to keep quiet rather than potentially expose themselves to shame or criticism. In observing instructors, I found examples of student deference throughout 
with greater student passivity and more disengaged behaviors in senior instructor classrooms than among younger instructors. I wondered whether student engagement had any applicability in a teaching and learning environment indoctrinated with cultural practices rooted in fear.

Independence. Many participants also stressed the value of independence, a finding that mirrored some of the theoretical propositions underlying student engagement and to some degree reflects the historical development of the KR itself. The university was viewed by participants as a prestigious institution, one to which students must earn admission through their demonstrated academic abilities. While the USAID-sponsored national scholarship test is used to identify government scholarship recipients and grant competitive admission to applicants, administrators conceded that those who receive the stipend and tuition waiver have little choice in the university they attend or the subjects they study. Students confirmed this with some stating they accepted the opportunity to study anything as they wouldn't have been able to afford otherwise. A student admitted, "I don't want to teach, but this scholarship was open. My parents are happy that I will have a diploma before I get married." For future teachers, administrators revealed that a three-year teaching contract at a public school must be completed to earn their diploma paper and graduate. Such ties of financial dependence were described as occasionally alienating student groupmates who were under contract with the university and could realize more independence in their immediate futures than those under scholarship.

The independence exhibited among practitioners whether in administration or instruction is also of note and a stated point of pride among some instructors. While some senior practitioners lauded their independent work and effort in attaining their 
positions, some junior practitioners voiced frustration at a culture of corruption and tribalism in which individual accomplishments had little impact upon promotions or opportunities. When viewed in this manner, the independence afforded practitioners with limited administrative oversight can be correlated with the maintenance of archaic theoretical curricula and the failure to develop functional and professional writing skills. Some junior practitioners admonished that there was little to incentivize furthering their skills as the university hierarchy consistently rewarded those with seniority or connections over those who published research, presented at conferences, or whose students succeeded. One admitted, "It is frustrating. Administrators select friends for fellowships. I know I am a strong teacher and my students learn and graduate. But awards and hours go to seniors and those of the same tribe." When students described some faculty as out of date, I interpreted that to mean old-fashioned or traditional. However, when students clarified their statements, they relayed that they felt that some instructors were expired and of little use to the university or its students. A student who was asked about their favorite instructor stated, "Very few are good. They younger teachers try. But many should go, they don't like teaching. It shows.” While such criticism would rarely, if ever, be voiced publicly it taps into some of the frustration and anger among students who are dependent upon the efficacy of a higher education institution and its practitioners.

Freedom. Finally, students and practitioners expressed a common perception that educators should be able to purposefully limit freedom through the restriction of behaviors, choices, or even opportunities. While students stated that they viewed their university experience as social, they also stressed the value that they placed on being able 
to interact with others enrolled at a limited access higher education institution. In-class, however, the permissive attitudes of students to one another lessened and many stated that they should do only as told by their instructor as an expert/specialist. Practitioners supported this belief as being in line with Soviet education with a student's purpose being to obtain and remember as much knowledge and information as possible. Limited freedom certainly has some value within a classroom in terms of compliance and management, however when learning a language, it could be viewed as counterproductive in some ways. Participants described curricula as being highly theoretical, not practical and viewed some English coursework as being focused less on communication and more on its linguistic mechanics and structure. For future teachers, translators, and interpreters, the disconnect in learning and in many cases memorizing antiquated theories pertaining to syntax and morphology rather than functional language is apparent. That learners would exhibit symptoms of being disengaged and passive when the curriculum ascribed to their program is significantly irrelevant is understandable and reflected in the student interview data. Several students echoed textbook concerns, "We mainly use Araken. The book is from Russia and is fifty years old. It is what the teachers learned so they still use it, but it is old, only theory. I cannot learn English from it." In addition, by cultivating respect for limited freedom, this can be interpreted as a means of promoting a culture of corruption. One practitioner revealed that they wondered why students were not more upset about their education and questioned why they preferred to keep quiet in light of instances of bribery and corruption disguised as university policy and practice. They remarked, "I see students sitting, keeping quiet, and I want to shout. This is their education and they aren't active 
with anything." Participant support of instructional practices that promote limited student freedom was described as being endemic to promoting status quo operations and outcomes at the university. Perhaps the explicit restriction of freedom could be interpreted as simply antithetical to Western culture and higher education, but the acceptance and preponderance of this cultural theme at the research site was notable even as an external observer.

\section{Findings by Research Question}

In this section, I extract specific information from throughout this chapter and apply it to each of my research questions and sub-questions. Table 3 presents a crosswalk of the ethnographic themes, codes, and evidence sources used to strengthen the research question findings resulting from data condensation and display. I present the findings in terms of observations and participant quotes by research question in the concluding section of this chapter. I offer additional explanation of the findings in the proceeding ethnographic interpretation and discussion section in Chapter Five.

Table 3.

Crosswalk of Ethnographic Themes, Codes, and Research Sub-Questions

\begin{tabular}{lll}
\hline Ethnographic Themes & Codes & Research Sub-Questions \\
\hline Fear & At Home & SQ1, SQ3A \\
& Keep Quiet & SQ1, SQ3C \\
& Shame & SQ1, SQ3C \\
& Honor and Respect & SQ1, SQ2, S3C \\
& Deference & SQ1, SC3C \\
& Scolding & SQ1, SQ3C \\
& Strong Instructors & SQ2, SQ3A \\
\hline Independence & University & SQ1, SC3A \\
& Prestigious & SQ1, SQ3D \\
& Groupmates & SQ1, SQ3B \\
& Independent Work & SQ1, SQ2, SQ3B, SQ3D \\
& Theoretical Curriculum & SQ2, SQ3A, SQ3E \\
& Corruption & SQ2, SQ3A, SQ3C, SQ3D \\
\hline
\end{tabular}




\begin{tabular}{lll}
\hline & Archaic & SQ2, SQ3A, SQ3E \\
Independent Work and & SQ1, SQ2, SQ3A, SQ3B, \\
& Effort & SQ3D \\
& Out of Date & SQ2, SQ3A, SQ3B, SQ3C \\
& Inability to Write & SQ2, SQ3D, SQ3F \\
\hline Freedom & In-Class & SQ1, SQ3C, SQ3D, SQ3F \\
& Limited Access & SQ1, SQ3A \\
Limited Freedom & SQ2, SQ3B, SQ3C \\
Disengaged & SQ2, SQ3B, SQ3C \\
Passive & SQ2, SQ3B, SQ3F \\
Social & SQ1, SQ3A, SQ3E \\
& Theoretical, not Practical & SQ2, SQ3A, SQ3E, SQ3F \\
& Corruption as Culture & SQ1, SQ3A, SQ3C, SQ3D \\
& Soviet Education & SQ2, SQ3A, SQ3D \\
\hline
\end{tabular}

\section{Research Question: How do post-Soviet practitioners reflect on their professional} experiences and Western benchmarks of effective educational practice? This

overarching question draws from the experiences, observations, perceptions, and reflections obtained throughout the course of this study. As such, the findings for each sub-question are detailed below with interview data identified by participant group and classroom observations included where relevant. The discussion and interpretation of these findings is a focal point of the concluding chapter.

\section{Sub-Question 1: How are practitioner and student higher education}

experiences described? A striking number of commonalities were observed between and amongst the descriptions of higher education experiences obtained from the interviews of various individual participants and groups. A lead administrator recalled their undergraduate studies as encompassing, "memorization that never ended, lectures that never ended, and notetaking that never ended." This same administrator elaborated that the actual difficulty lay in finding materials needed to complete course requirements as English materials were in especially short supply during Soviet times. A senior instructor 
spoke of the camaraderie among students during her studies stating, "my groupmates were my family at university, many of us are still friends and now colleagues, we have grown up together." A young instructor stated, "my group had some troubles, but we helped each other when we could, a few of the boys never attended so it was mainly girls studying together in school." A current student elaborated that, "we have official groupmates and friends. Sometimes the same, sometimes different. Outside of school I am with family, university is separate."

\section{Sub-Question 2: How are effective educational practices among faculty and}

students defined? Participants collectively described 'strong instructors' when reflecting on the best instructors they encountered in their studies. Classroom observations indicated that university instructors were very strict in terms of appearance and professional conduct. However, it was noticeable that younger instructors tended to interact with students more in the classroom and deviated from a standardized lecture class format. A senior faculty member described the best teachers by indicating, "they have control of the content and the room. They are experts in their subjects and know the facts. They help train new specialists in subjects." One junior faculty member added, "The best teachers are active in their area. They publish, write, and speak on subjects. They show students how to learn and how to grow in university." One student remarked, "The best teachers care about their work. They know what they are teaching. It shows."

\section{Sub-Question 3: How are the NSSE benchmarks and their practical potential}

interpreted? This overarching question was investigated via interview and observation of each individual benchmark among participants and within classrooms in the teaching 
and learning environment of the university program. Findings are presented by subquestion and benchmark below.

Sub-Question 3A: How challenging is this academic program? The most significant challenges observed to be endemic to the program concerned logistics and facilities. When the already limited technology would malfunction, leaving classrooms in darkness and computers nonfunctional, instructors and students were challenged to continue their studies. In one instance, a class was conducted via cellphone flashlight with students taking notes from a lecturer they could barely see. Participants were united in stating that the difficulty of the academic program had lessened relative to previous years or Soviet times. Administrators placed the decline in academic challenge on new accreditation standards, the Bologna process, and less prepared student populations. Instructors attributed the decrease in challenge to condensing study to four years and the loss of many senior instructors to retirement and relocation. Students stated that academic challenge depended on the instructor with one stating, "an easy course can be difficult if the teacher doesn't cover the material on the exam or expects something for an exam mark." These subtle references to corruption described as cultural practice were found throughout references to academic challenge in the program.

Sub-Question 3B: To what extent is learning active and collaborative? Practitioners and student responses varied somewhat on this sub-question. Administrators admonished that learning should be active and collaborative throughout the university but wasn't in all courses. Faculty claimed to use methodologies supporting active and collaborative learning, but students stated that most senior faculty only lectured or gave presentations. Classroom observations revealed notably quiet learning 
environments conducted in a traditional learning environment. While younger instructors were observed to employ some student-centered learning techniques, most classes were direct lecture with instructors providing information and students taking notes.

Sub-Question 3C: In what ways do practitioners and students interact?

Classroom observations supported limited interaction between practitioners and students throughout the learning environment, even in the hallways. While physically together in classrooms, students were observed to ask permission from the instructor to leave the room for any reason and only spoke if answering a question or when called upon.

Administrators and faculty remained in their respective offices when not teaching, and students acknowledged that they would not enter unless they had an appointment. Participants acknowledged that this was standard practice with limited interaction outside of the university and formal interactions on largely academic matters within classrooms and offices.

Sub-Question 3D: To what degree is the campus environment supportive? University observations indicated that student support services existed in name only, although practitioners claimed that the university had more established facilities and resources dedicated to student support. Students stated that while the university officially described such services, the medical school and other departments with large international student populations were the only ones to allegedly have them. Younger instructors reported counseling students, even on personal matters to help them finish their diplomas. However, senior instructors and administrators were quick to point out where their responsibilities began and ended. One directly stated that, "we are here to 
teach, students are here to learn. It is their choice to start a family or be absent. It is not my fault if they fail."

\section{Sub-Question 3E: How enriching are higher education experiences?}

Opportunities to study abroad or complete field experiences were observed to be supported by practitioners but driven by students and organizations external to the university. Administrators took great pride in referencing alumni and faculty who had secured opportunities to study abroad. Faculty also referenced successful students who found ways to live, study, and work in other countries, often mentioning the amount of money they earned in passing. Students candidly stated that the university did little to inform them of opportunities and sometimes created problems in securing required documents and letters. While the diversity component of this benchmark was observed to be present with students from many nationalities and backgrounds in attendance.

Discussion and acknowledgement of these differences among students was discouraged if not restricted by administrators and faculty within the learning environment.

\section{Sub-Question 3F: How might the NSSE benchmarks impact praxis and quality?}

Classroom observations indicated that basic university infrastructure is lacking and existing campus resources, including instructional human resources, are nearing capacity. The feasibility and practicality of obtaining the resources needed to improve the campus environment or add enriching educational experiences to undergraduate studies was observed to be unlikely given the declining conditions of existing university facilities. Administrators and senior instructors agreed that some of the benchmarks were already in use, just not by name and that further implementation would require too much money and time. Junior instructors conceded that without funding or additional time and training it 
was unlikely that additional tasks would be completed by instructors. In addition, they stated that some senior instructors would be hesitant to embrace such major changes given their established practices. Finally, students voiced doubt that the practices were being used as they had failed to encounter them in their time at the university. They also expressed doubt as to whether they would work here given the cultural differences between the United States and the KR with one student quipping, "more no than yes."

\section{Summary}

This chapter presented the findings from conducting qualitative field research at the study site. I used data condensation to compile the ethnographic codes via description and performed subsequent data display analyses before describing and presenting them in sequential order. First cycle codes obtained through descriptive, invivo, and values-based coding procedures consisted of university, at home, in-class, prestigious, limited access, groupmates, keep quiet, shame, independent work, theoretical curriculum, corruption, limited freedom, and honor and respect. Classroom observations yielded in-vivo codes consisting of passive, disengaged, archaic, and deference. Second cycle codes derived through in-vivo and values-based coding included social, scolding, limited freedom, strong instructors, independent work and effort, theoretical not practical, out of date, inability to write, corruption as culture, and Soviet education. A narrative analysis displayed each code in context, a checklist matrix focused upon the presence or absence of key NSSE benchmarks, and participant vignettes highlighted defining individual experiences representative of the teaching and learning environment under analysis. Three ethnographic themes emerged from the data condensation, display, and analysis processes: fear, independence, and freedom. The findings were then presented 
by research question and sub-question with reference to direct findings and sources of evidence. In the final chapter, I provide a cumulative ethnographic interpretation of thematic findings by research question and posit implications and recommendations for higher education policy, practice, and research. 


\section{CHAPTER V}

\section{SUMMARY, INTERPRETATION, IMPLICATIONS, AND CONCLUSIONS}

This chapter concludes the study by first providing a summary of the research process and findings including the predominant ethnographic themes. I then discuss the research question findings presented in Chapter Four. Next, I present the final ethnographic interpretation stemming from the study and focus on developing meaning, drawing conclusions, and verifying those conclusions. I then review implications and recommendations derived from study findings with a focus on policy, practice, and research; in doing so I reflect on my own position in the study and the lessons learned while conducting qualitative fieldwork. The concluding section discusses the holistic process comprising the development and execution of this study, imagines its potential impact, and provides a reflective analysis of the dissertation process.

\section{Summary of Study}

This study emerged from my professional fellowship experience in higher education at the research site and stemmed from a review of the extant literature on the Kyrgyz Republic and student engagement. The study attempted to develop a greater understanding of the experiences of students and practitioners at the research site, the realities of post-Soviet higher education, and perceptions of Western benchmarks of effective educational practice. As such, the study filled gaps in the existing literature by using NSSE benchmarks as a theoretical framework for understanding higher education 
practices and experiences in an institution in this environment. The study also explored a greater conceptual topic: the challenges of translating evidence-based practices developed in one context into a practical context that is markedly different. As an ethnographic qualitative case study, I analyzed information obtained from within the teaching and learning environment at the host university in the KR. Qualitative data consisted of semistructured interviews with practitioners and students as well as multiple classroom observations. In the content analysis, I used first cycle and second cycle coding procedures to elicit my findings. I interpreted these findings by situating them within the realities of life and learning for those at the host university and drawing from current student engagement research. I did so to further the potential for this study to contribute to improving the quality of post-secondary education via effective learner and practitioner practices.

\section{Summary of Findings \& Ethnographic Themes}

After each of the three data collection stages, I first transcribed and then analyzed the data using first cycle and second cycle coding procedures. I assembled the findings into an ethnographic case description to further development of the thematic ethnography and ethnographic interpretation. Throughout data collection, analysis, and interpretation, I strove to maintain the authentic voices of practitioners and students both to preserve their contributions and strengthen the study. The detailed analysis of emergent ethnographic codes and findings from the previous chapter indicate the significance of cultural themes pertaining to fear, independence, and freedom in the teaching and learning environment at the research site. These themes act in concert to illustrate how 
practitioners and students in this program reflect on their professional experiences and Western benchmarks of effective educational practice.

\section{Discussion by Research Question}

In this section, I discuss the ethnographic themes, codes, and evidence sources used to strengthen the research question findings resulting from data condensation and display. I offer additional discussion of the findings in the proceeding ethnographic interpretation and discussion section in Chapter Four.

\section{Research Question: How do post-Soviet practitioners reflect on their} professional experiences and Western benchmarks of effective educational practice? The professional experiences of practitioners and the learning experiences of students in the program mirror one another in several ways. Practitioners reported greater academic challenge and time spent preparing for courses compared to their students. In turn, students supported the claim that they spent little time in preparation for coursework, however they countered that the level of academic challenge they encountered was more closely related to individual instructors and their practices, rather than actual content difficulty. While each group reported some active and collaborative learning among their groupmates, the interview data indicates that this was both more common and functionally necessary in previous years. Student-faculty interactions were described as existing along formal lines with indicators of bribery and corruption within both generations of learners. The concept of a supportive campus environment was foreign to most interview participants with practitioners describing some support services but firmly placing responsibility upon students for their learning and outcomes. Students claimed that such academic services were non-existent and that they were on their own in terms of 
overcoming difficulties or injustices encountered as undergraduates. Finally, in terms of enriching educational experiences, commonality exists among practitioner and student perspectives in that racial/ethnic/political/social/religious diversity is not forthrightly recognized and the suppression of certain topics in the past correlates with hesitance and avoidance in the current learning and teaching environment.

\section{Sub-Question 1: How are practitioner and student higher education}

experiences described? Students and practitioners reported higher education experiences that were generally more similar than different. Both groups of participants described regimented instruction, pervasive corruption, and a primary reliance on themselves and their fellow students or groupmates. Administrators and senior practitioners reflected on Soviet education with pride as they described overcoming barriers to simply gain admission to a prestigious university. Once in university, they viewed completion of their program and receipt of the diploma as essential guarantees for a career and livelihood provided they attended and passed exams, or financially supported instructors and the university. Practitioners from the post-Soviet era described their experiences in slightly different terms in line with turmoil of the KR transition to a democratic republic. While strong instructors who rarely displayed emotion or empathy were feared, they were also respected as experts or specialists in their field, and they viewed their efforts and outcomes as students as being largely dependent upon themselves. However, practitioners lamented that the assurance of a career for those who obtained a diploma vanished with the collapse of the Soviet Union as did some of the strongest instructors and researchers. Students portrayed their undergraduate experiences as an exercise of deference to instructors in class and practitioners in the university in keeping with the 
wishes of their parents. The avoidance of shame by elders as described by students, led many to keep quiet despite blatant corruption and out-of-date curricula and practices. That several students could not name the degree program they were in or the department in which they were enrolled is emblematic of the extent to which they are disengaged within the university environment.

\section{Sub-Question 2: How are effective educational practices among faculty and}

students defined? According to faculty and students, the best instructors demonstrate strength in their subject matter-in this case, they are experts or specialists in the English language. Participants described honor and respect being bequeathed upon elder, experienced instructors, despite their practices and content remaining highly theoretical, archaic, and in some instances incorrect. Interactions with strong instructors are described as reverent both for their status at the university and their expertise in English, notably without regard to their instruction or skill. Strength as an instructor was described by some participants as effectively limiting student freedom and maintaining their independence and predominance within classroom instruction. Students and junior practitioners thoroughly respected senior instructors and administrators although undertones of disenchantment with curricula which do not reflect the realities of work as an interpreter, translator or teacher. Participants lamented a lack of practical program content which would foster skills of value to students in the future. Of equal concern to administrators, instructors, and students alike was the fundamental lack of content related to teaching methodology and writing, with deficits remaining despite multiple internal and external efforts.

\section{Sub-Question 3: How are the NSSE benchmarks and their practical potential}


interpreted? Administrators viewed the benchmarks as practices that should be used while acquiescing that they provided little classroom supervision and doubted the potential allocation of time and resources to implement the benchmarks. Most instructors stated that the university and this program essentially already uses the NSSE benchmarks, though not by name and apart from writing practices. This was especially evident among younger practitioners who embraced communicative language instruction and projectbased learning while elder practitioners shirked the benchmarks as being theoretical, not practical. Students generally disagreed, perceiving that in many ways, the university experience remains thoroughly Soviet in nature and that the program does not follow many of the benchmarks, lacks quality, and fails to meet their expectations. As an external observer, I argue that the nexus between the NSSE benchmarks and improved quality practice is most plausible for younger practitioners and students as indicated by their willingness to discuss student engagement and apply it within their instructional practice.

\section{Sub-Question 3A: How challenging is this academic program? Administrators} lamented that the quality of students admitted to the university jeopardized its prestige and that limited access through higher tuition might improve student performance. Practitioners viewed the program as challenging, but less so than during their own studies as resources more accessible and examination formats have changed. Students view the program as challenging because they face additional obstacles outside of the classroom and lack administrative oversight of faculty and university support in general. The reality conveyed by participants from throughout the university is that following admission, graduation is essentially guaranteed provided a student contributes the proper amount of 
money, regardless of effort, attendance, or performance. In addition, a diploma no longer equates to a career or improved social status, and work at a school or university as a teacher is often insufficient to support oneself, much less a family. As an observer, I view the program content as incredibly challenging given its archaic complexity and that the language of instruction may not be understood by learners on a functional level. When asked my opinion by a student participant of the program at the university I remarked that, "it is well-intentioned, but no matter how hard anyone tries, it is impossible to teach English in Russian."

Sub-Question 3B: To what extent is learning active and collaborative? The nature of learning within the program depends largely on individual instructors and their coursework. Practitioners control their classrooms with limited oversight, and students heed their course instructors' policies and procedures regardless of their coherence or applicability. Classroom observations revealed little in terms of student activity or collaboration with attendance being the hallmark of the educational process. Those practitioners who employed project-based learning or attempted discussions with students were observed to need to scaffold such efforts to a tremendous degree and outcomes in terms of successful project completion remained minimal. In reviewing the classroom observation data, I recall several times where absolutely nothing took place, the teacher stood, the students sat, and I wondered whether I had missed something. In reflecting, I recalled a quote ascribed to K. Patricia Cross, "Teaching, without learning, is just talking," (Angelo \& Cross, 1993, p.3). I wondered whether teaching without talking or learning, as I observed, was just standing. Further, in some classes it appeared that 
attending, without teaching, without learning, and without talking, was de facto educational practice.

Sub-Question 3C: In what ways do practitioners and students interact? While some practitioners indicated that they reach out to build relationships with students beyond the classroom, descriptions of limited professional and traditional student-faculty interactions predominated interviews. Administrators were observed to remain in their offices and limited their access to faculty and students alike; several students were unable to name the dean or the president of their university. Student interviews indicated that they were unlikely to voice problems outside of the university that impact their studies to groupmates, much less practitioners. In addition, students admitted that faculty interest and interaction often peaked around examinations, when suggestions for gifts were often floated tacitly. As an observer, little student-faculty interaction was observed even within the classroom, and in some it felt as if the instructor had wandered into a room full of waiting students as class start times and punctuality were also decidedly fluid. Markedly little oral or written feedback on assignments or performance was observed, and several participants revealed that a lack of feedback and questions with very narrow acceptable answers was a tenet of Soviet education. Again, instructors appeared to have the power to determine both the quality and quantity of faculty-student interactions and practitioners and students alike admitted that younger faculty were more likely to engage.

Sub-Question 3D: To what degree is the campus environment supportive? Administrators insisted that the university supports its students and provides academic services for their success. While practitioners believed that such facilities and services were in place, aside from a class feedback form, few specifics were provided via 
interview. Meanwhile, students contradicted these statements, and my observations likewise failed to uncover any initiatives to promote a supportive campus environment beyond those organized by students. In reviewing the participant vignettes, it is evident that students are very much alone in dealing with their problems as the hierarchy of the university and impropriety of voicing criticism are apparent. Practitioners viewed the institution as being separate from students' personal problems and issues, generally believing that it was an individual's choice and responsibility to meet the requirements for a university degree. Students often cited practical considerations that impacted their efforts to study such as a lack of money to travel to school, recurrent illness, or domestic problems. While groupmates were a degree of support, the social aspect of university as defined by independently organized student events including small-scale plays and festivals for traditional holidays were clearly sources of pride in some interviews.

\section{Sub-Question 3E: How enriching are higher education experiences? The} kindness and openness of interview participants encountered a noticeable stumbling block in broaching the content of the final benchmark. Senior practitioners kindly but firmly refused to discuss questions oriented around practices associated with race/ethnicity/nationality, much less politics or religion. One practitioner explained that such differences were suppressed in Soviet times and that the university restricted political and content and discussion, especially within the classroom. Even younger practitioners and most students exhibited hesitance to address such topics with many asking for definitions to race, ethnicity, and nationality. I interpreted this hesitation as being representative of the inevitable barrier between myself as an external researcher and participants within a shared university and culture. In addition, recent ethnic clashes 
and heightened religious tensions throughout the region made such discussions extremely sensitive, and I elected to focus on external learning experiences. Students were candid in their opinions of community service and volunteer work, explaining that in their culture, people do not appreciate or understand the concept of working without being paid. Students expressed interest in completing interpretation/translation internships or field experiences however many felt that the university should be responsible for organizing such things as some had been turned down by local organizations. Practitioners reported that previously students had to complete an active teaching practicum at a local elementary school while an optional observational practicum exists today for logistical reasons. Finally, while opportunities for study abroad and travel abroad are now possible, participants reported that most must be fully-funded to be even remotely plausible and a few remarked that, even now, the most qualified candidate is not necessarily selected. I interpreted this statement as another consequence of corruption with a plausible connection to students feeling disengaged and even disenfranchised.

\section{Sub-Question 3F: How might the NSSE benchmarks impact praxis and quality?}

Senior practitioners viewed the benchmarks with noted skepticism and remarked that most were already being used, just not by name. Junior practitioners were a bit more forthcoming in admitting that often what they plan and do are quite different and that the benchmarks are nice ideas that would take a lot of time. Students posited that even if the benchmarks were required to be implemented, some instructors would keep their same lectures and content in place, a statement generally supported by practitioners. Participants revealed that practices associated with creating a supportive environment and providing enriching educational experiences were most out of line with current praxis in 
the university. When questioned further, participants identified the critical analysis and writing components of academic challenge as good ideas that were problematic to use in class. Practitioners acknowledged significant professional capacity deficits in writing and research, attributing both to cultural differences and skills lacking from their own educational backgrounds and training. Practitioners specifically emphasized building research capacity to improve praxis and academic quality, while students called for more educational experiences and practical opportunities that will prepare them for employment or further education. From my perspective, the most urgent capacity gap among practitioners and students is writing. While a practical training focus on developing writing skills might be viewed as simplistic when compared to the overarching student engagement construct it harbors the potential to fundamentally alter the quality of the undergraduate experience in this program by advancing an essential language skill.

\section{Ethnographic Interpretation and Discussion}

I applied a holistic view to interpret this study's findings on student engagement for application in higher education research and practice. Kahu (2013) viewed student engagement as a combination of behavior, emotion, and cognition with university precursors and multiple results all influenced by sociocultural forces. Similarly, the ethnographic case in this study sits within a functioning but strained educational system with internal and external forces that are constantly changing. The extant literature describes the KR itself as a unique political and social microcosm of post-Soviet culture, power, policy, and economics compounded by relative geographical isolation. The sociocultural components of life in this region are reflected in the personal interviews I 
conducted. The impacts of culture among the individuals comprising the university is evident on a foundational level within the study data and explains many of the educational practices and standards in place. There is room for improvement in terms of both student outcomes and instructional practices, a reality admitted to by practitioners and students alike. Such reform efforts in a transitioning educational context where resources are in short supply must be especially cognizant of what realistically can and cannot or should and should not be changed (DeYoung, 2005).

In the learning environment analyzed in this study, interviewed instructors admitted that they possess a significant degree of control over how they conduct their classes, what content they teach, and how much money they earn. Administrators elaborated that there is little oversight of faculty instruction due to the large number of instructors, increasing numbers of students, and space-restricted class sizes. In interviews, students relayed that while there are surveys at the end of courses, there are no academic support services or grievance procedures. While this statement runs counter to practitioner assertions that such services and procedures exist; all interviewed populations admitted that student criticism of instructors is rarely expressed. As an external observer, the relative independence and freedom of practitioners presented a plausible opportunity to improve student outcomes via classroom instruction quality through the introduction of effective practices associated with student engagement. I postulated that to be able to leverage student engagement to promote meaningful and sustainable change, practitioners would need to have the skills to evaluate the concept, apply it in practice, and eventually improve student outcomes. To do so, I constructed this study around Schön's reflective practitioner model in order to ascertain the degree to 
which practitioners would be willing and able to reflect on their own university experiences, understand student engagement and its relative practical value, and incorporate it into their instructional practice.

This study applied the NSSE benchmarks as the theoretical framework and a means for analyzing the learning and teaching environment at the research site. In addition, Kuh's (2002) theoretical propositions underlying the benchmarks inform my interpretation of the findings relative to student engagement. For example, the interview data revealed that participants value independent work as being essential to learning and success, with the onus increasingly placed on students to complete assignments and identify opportunities for growth on their own. This aligns with the student engagement proposition that what students do during university matters more than who they are or where they attend (Kuh, 2002). Unilaterally, students cannot change who they are, and participant interviews revealed that scholarship students in this ethnographic case also cannot select where they attend or what they study. In light of this revelation, I interpreted my observations of students as passive and disengaged as being reflective of their general lack of freedom of choice and independence concerning their postsecondary studies. Restated, I developed a greater understanding of why learners would not engage with archaic classroom material at universities and in subjects for which they had no part in selecting or interest in general.

However, there is a significant amount of research indicating that who university students are and where they intend do matter in terms of educational attainment and other measures of success. The most recent report from the National Student Clearinghouse Research Center presents data indicating that while overall college completion rates have 
risen in the United States significant disparities remain among student cohorts divided by race and ethnicity, who students are, and institution type, where they study (Shapiro et al., 2017). On a more global scale, Thelin observed that life choices are probabilities, and earning a college degree takes more than talent and dedication (2007). The probabilities and challenges associated with higher education in the KR further compound matters as participant interviews revealed that cultural practices within some traditional homes may prohibit further study completely. The first participant vignette in this study reflected on the experience of trying to complete a degree: "it absolutely depends on your family and the support that you have; our culture is different." Participants also indicated that the preponderance of corruption as a cultural practice throughout higher education also restricts some students from completing their studies despite their academic effort and accomplishment. Simply put, the interview data indicates that at this institution, students can graduate without trying or even attending for the right amount of money, while others who try and attend may be unable to receive their documents until they contribute enough money. In the end, students can overcome some barriers, but not all of them.

Scholars have advanced the proposition that good institutional practices yield student engagement and that engagement in turn fosters student success and can be equated with academic quality (Kuh, Kinzie, Cruce, Shoup, \& Gonyea, 2007). I view student engagement's link with academic quality as being dependent on the definition of both concepts as well as the variables being used for their assessment. Also, the logical premise underlying the link relies on the assumption that one necessarily leads to the other in higher education reform; this can be viewed as circular reasoning, specifically petitio principii, begging the question. This study presented an opportunity to evaluate 
the contrapositive: that negative practices do not yield student engagement nor academic quality. Participants confirmed that the institution essentially permits plagiarism. In applying the appropriate NSSE benchmark and practices, the number and lengths of papers written cannot plausibly correlate with the perceived level of academic challenge or student engagement or academic quality. Even if student engagement as defined by the benchmarks were present at the research site, the ability to write, especially as a translator, is a valuable student outcome and forms a logical basis for academic quality in this program. A renewed focus on good practices linked with valued student outcomes, even if they are not necessarily directly linked to student engagement, has the logical and practical potential to positively affect the undergraduate experience and academic quality of this program.

This study finds a strong degree of correlation between practitioner and student reflections and perceptions of their teaching and learning experiences in this program. Strong instructors are defined by study participants as experts or specialists, meaning that students and faculty members value the knowledge an instructor has in their subject matter over their selected teaching methodology or classroom practice. Similarly, strong students are defined by the results of their independent work, by what they accomplish and learn over the course of their studies. Participants viewed the NSSE benchmarks as good practices but conceded that their implementation would require additional instructor time that does not exist and most likely cannot be funded. From my perspective, student engagement was evident in the number of university students who reached out to me-in their third or fourth language — and offered to tell me their story and share their experience. I believe that the quality of higher education and student outcomes in this 
context depend on a constellation of factors and variables, a host of which cannot be controlled. To promote positive change within this program, practices must be geared to the specific realities of education at the research site identified throughout this study. When asked whether the benchmarks had practical potential to improve academic quality at the program, most participants responded in the negative, and to quote the student participant vignette, "more no than yes."

\section{Implications and Recommendations}

As an ethnographic qualitative case study, the findings have restricted generalizability. However, the findings can inform future efforts and potentially guide practice, among other purposes. In the following paragraphs, I draw on evidence from the study to discuss theoretical implications and practical recommendations for the field of higher education.

Policy. Donor organizations, including international aid agencies, foreign governments, charitable foundations, and non-governmental entities, provide funds that are essential for maintaining basic educational services in developing and transitioning nations. While such policies are ostensibly well-intentioned, donor organizations should fully disclose the stipulations attached to funding and the agendas supported by it. At the research site, many practitioners and students were surprised to learn that the United States Agency for International Development (USAID) partnered with the KR to develop its national scholarship test. Few participants were able to think critically about why USAID would use American taxpayer dollars to support such an initiative.

The blatant perception of international aid as free money may damage initiatives just as much as funding tied to an outright agenda. The KR maintains numerous 
programs with educational funding from organizations as diverse as the KfW Development Bank, the Aga Khan Fund, and the Open Society Foundations. It is critical that beneficiaries understand the source, purpose, and terms of funding to avoid potentially destabilizing political entanglements and conflicts of interest (Satke, 2017). In addition, beneficiaries should prioritize initiatives that offer fiscal and operational independence so that the funded objectives can be long-term and large-scale—-for example, educational quality improvement (Satke, 2017). By coordinating efforts among various non-governmental and governmental agencies, the KR could reduce the risk of contradictory or duplicitous initiatives and could complement internal programs and objectives. Unfortunately, this coordination seems unlikely in the KR at present given political instability and questionable rule of law.

The United States also has a responsibility to promote greater cognizance among beneficiaries of donor organizations. Recipients who recognize the underlying agendas of philanthropic corporations and organizations might be empowered to assume more active roles in programs that impact their communities. Raising such awareness and fostering such empowerment could translate into more nuanced efforts from entities such as the Chan Zuckerberg Initiative and the Bill \& Melinda Gates Foundation as they search for effective means to connect theory to practice and outcomes to alleviate persistent educational problems.

Practice. The previous section suggested that several philanthropic organizations have failed to improve educational practice in the United States despite ostensibly good intentions, thorough research, adequate funding, and expert implementation (Freedberg, 2017; Callahan, 2017.; Gates \& Gates, 2018). It should come as little surprise, then, that 
less-coordinated educational reform efforts in the KR have failed to evince lasting positive change for educators and students (Brunner \& Tillett, 2007; DeYoung, 2005;

Gita, 2016). This study demonstrates that it is challenging to evaluate the potential efficacy of evidence-based practices before they are ever implemented. On a conceptual level, this study advocates for understanding the differences between the developmental context, where theory-driven research generates evidence-based practices, and the practical context where those practices will be implemented. Before experts and specialists advocate that practitioners implement new educational practices, they should analyze contextual and cultural factors that may impact their support and efficacy.

On a theoretical level, this study used NSSE benchmarks to explore the teaching and learning environment within the case. I found that some of the benchmarked practices are already in use; thus, the institution promotes student engagement to some extent without naming or recognizing it. On a practical level, the burden would fall on instructors to adopt additional student engagement practices because the students lack empowerment and the university administration provides little direct management.

Restated, the evidence from this study indicates that the successful implementation of externally developed evidence-based classroom practices is dependent on practitioner support. It seems unlikely that practitioners will receive the additional resources, time, and training to implement the benchmarks given the current higher education funding structures and the failure of previous reforms to change university instructor compensation (Gita, 2016). I posit that practitioners at the research site are more likely to succeed in improving academic quality if they foster the changes from within by raising 
awareness of existing campus support services (if they exist), prompting the university to incentivize research efforts, and promoting writing as a skill-based focus of curricula.

Unfortunately, this study finds that practitioners are constrained in many ways by external and internal forces that are at once poorly understood and highly variable. Within the university as it exists today, the leadership lacks the transparency needed to reassure practitioners of their roles and support. I charge that educational practitioners and reformers interested in improving academic quality in the KR need to conduct additional research looking within organizations to assess the potential of initiatives prior to implementation as attempted in this study. Foundations seeking to improve academic quality in the United States are increasingly seeking to foster continuous educational improvement using locally-driven practices and reforms oriented around professional capacity development (Freedberg, 2017; Gates \& Gates, 2018 ). Reformers may be tempted to implement such practices in the KR based on research-guided empiricallybased evidence. This study demonstrates that there is a sound argument for conducting research among stakeholder prior to implementation to gauge potential effectiveness and efficacy at the site of implementation. For example, current mechanisms of university funding and faculty compensation in the KR were observed to limit the institution's ability to emphasize quality over quantity in instructional and organizational practices. This has the potential to negate even the most promising reforms given the financial realities surrounding continuing to operate basic university functions in the KR.

To tacitly promote greater alignment with the NSSE benchmarks and hopefully improve functional student outcomes, the university should emphasize writing skill development among staff, faculty, and administrators. I argue that this final 
recommendation has the most potential to improve the quality of the teaching and learning environment in advancing positive student outcomes.

Research. The lack of accessible, reliable, and valid quantitative data at the research site restricted the scope of this study. I recommend rigorous quantitative studies to further substantiate qualitative observations and program-based evaluations with a goal of developing a greater understanding of educational issues in the KR. If future researchers could gain access to national scholarship databases, they could analyze a plethora of quantitative data on the academic skills and performance of KR students at the completion of secondary school. Quantitative action research could prove extremely valuable to participants as well; the region lacks the professional research capacity to conduct such research internally. Such research could also foster data analysis skills development, thereby bridging gaps in capacity and practice. Additional qualitative investigations could develop nuanced perspectives on teaching, learning, and the roles of ethnicity and nationality in promoting or inhibiting educational quality throughout the post-Soviet KR. The combination of qualitative and quantitative research methodologies would improve the practicality and generalizability of future research on educational interventions and quality improvement.

The student engagement construct remains contentious within higher education research, and several researchers have called for additional exploration of its components in varying contexts (McCormick et al., 2013). Follow-up qualitative investigations of NSSE results may negate or substantiate criticisms of both the instrument and the overarching construct. In-depth explorations of university student engagement may realign time-sensitive aspects of the construct with current higher education practices, 
beliefs, and values. I call for researchers to critically examine the efficacy of student engagement as an indicator of academic quality, especially considering its increased use as a performance-based funding measure. Additionally, higher education researchers should strive to include greater diversity and inclusiveness, even on the basis of geography alone, to improve the generalizability of findings in an increasingly globalized higher education marketplace.

Further research in post-Soviet higher education should also clarify student expectations for practitioners and the university experience. Students in the present study stated that their expectations were not being met, but they struggled to express precisely what those expectations were. Similarly, it is yet unclear how students and faculty define success and failure. In a higher education system where essentially all paying attendees receive a diploma, what defines a successful or unsuccessful university student? Practitioners voiced concerns over the constraints and difficulties under which they teach and administer, but I did not examine interventions that might make their roles easier. Finally, future research should construct and define the overall educational philosophy of the KR. After thirty years of independence, the existing body of literature indicates that post-Soviet higher education systems throughout Central Asia remain largely Soviet in both principle and practice. Arguably even the most visionary educational changemakers cannot develop and modernize the system until they closely examine and understand the fundamental components of education within the post-Soviet KR.

At a very granular level, additional targeted research should be conducted to ascertain the purpose of this higher education institution in the KR and this program in the community. Participants struggled to identify the role of the university or the 
program in the modern KR citing outdated curricula and limited career prospects. This raised questions beyond the scope of this study such as: if the university functions to train the next generation of language teachers, then why are students grouped together with the translation and interpretation department and why is there limited methodological or practical teacher training? On a larger scale, does the university intend to promote opportunity and social advancement or does it function to preserve the status quo and as a result reproduce inequality in society? Answering such questions would require extensive interviews with program stakeholders as well as community members and university leaders. This would also require a fair amount of interpretation as it is unlikely that interviewees would directly voice harsh criticism of a program, particularly if they are dependent upon it for their education or livelihood.

Implications for Student Engagement. In reviewing the higher education literature pertaining to student engagement, it became increasingly evident that researchers' interpretations vary significantly in transitioning between and amongst concepts to theories to practices to outcomes. A special issue of The Review of Higher Education was dedicated to critiquing student engagement and NSSE in particular (Olivas, 2011). In providing an overview of the critiques presented within the issue, Olivas (2011) cites articles targeting the validity of the NSSE benchmarks, lack of theoretical justification and vague item inclusion justification, questioning the validity of self-reported data and college student survey questions in general, the inter-correlation of variables and lack of predictive validity for the benchmarks relative to important outcomes, generally poor reliability and validity features of the benchmarks as measures of institutional effectiveness, the accuracy of the factor structure, and the lack of 
individual institutional data supporting benchmark validity (Olivas, p. 1-15, 2011). Similarly Higher Education Policy released an issue entitled "Critical and Alternative Perspectives on Student Engagement," Macfarlane and Tomlinson (2017) cite articles critiquing student engagement on grounds of performativity, marketing, infantilization, surveillance, gamification, and opposition before presenting critiques of student engagement 'learnification,' the strength of its policy agenda, associated student agency, lack of a student-centered approach, the ethical implications of assessing learning via student engagement, and the association of student engagement with excellence within a transactional model (Macfarlane \& Tomlinson, p. 1-4, 2017). In a nutshell, there are surprisingly few direct sources of evidence for the NSSE benchmarks to be lauded as evidence-based student engagement practices associated with academic quality. The student engagement construct itself remains highly conceptual and theoretical with ongoing philosophical debates throughout the literature regarding its metaphysical basis and componentry. I was drawn to a related passage by Jack London in The Iron Heel (1907):

In short, the metaphysicians have done nothing, absolutely nothing, for mankind. Step by step, before the advance of science, they have been driven back. As fast as the ascertained facts of science have overthrown their subjective explanations of things, they have made new subjective explanations of things, including explanations of the latest ascertained facts. And this, I doubt not, they will go on doing to the end of time. (p.7)

McCormick and McClenney (2011) composed a rebuttal to the original special issue arguing that the benchmarks were developed with consequential validity for subsequent decision-making, that the benchmarking process is one of continuous improvement, that its primary use is heuristic and intended for practitioners, that there is value in asking students about their experiences, and that the fundamental purpose underlying NSSE is to 
promote improvement by highlighting educational practices associated with good student outcomes (McCormick \& McClenney, p. 307-333, 2012).

To once again quote Ernest Everhard in his metaphysical argument with Dr. Hammerfield in The Iron Heel, "That's another way of saying that you live up in the air. But you come back to earth at meal-time, I am sure, or when an earthquake happens along," (London, p.10, 1907). I argue that student engagement practices as effective means to an academic quality end remain 'up in the air' and that its perception as a panacea for institutional improvement is debatable if not worse. A key consideration which has yet to be fully addressed relative to the ongoing debate about student engagement on both conceptual and practical grounds is that it has made the careers of several individuals and generated sufficient revenue to become a 'self-supporting auxiliary unit' within the Center for Postsecondary Research in the Indiana University School of Education ("NSSE About NSSE," 2018). In the words of Ernest Everwood, student engagement is the 'bread and butter' for more than a few academics, and some have clearly vested interests in promulgating the idea, its assessments, and associated practices regardless of their efficacy or validity within institutional effectiveness. While ostensibly there is nothing wrong with academics profiting from their pursuits, I charge that the scale of NSSE within higher education research and practice and its promulgation within the global higher education marketplace as a quality improvement product and academic standard raises fundamental ethical concerns which must be evaluated. While the literature indicates recent critical analysis of the student engagement construct and its practical applications, the vehement defenses by some academics could be viewed as somewhat biased when taking into consideration potential conflicts of interest. 
Theoretical editorialization aside, the reality is that students could potentially benefit by improving the quality of education in this program and that the evidence indicates that there is substantial room for such improvement. I argue that practical concerns for the continued operation of the program at the research site outweigh the potential benefits associated with implementation of the benchmarks to improve academic quality. In addition, the lack of direct ties to subject matter knowledge and practice are too abstract for reform efforts at the research site. I posit that discrete, site-specific, targeted initiatives to develop much-needed academic writing skills among practitioners and students have more potential than those attributed to student engagement.

Researcher Positionality and Reflexivity. Since I previously lived and worked at the research site as a fellow, I thought I had a general understanding of higher education in this region before launching the present research study. The experiences of practitioners and students surprised me in some ways, and yet admittedly met my expectations in others. From my perspective, the function of university in this culture approximates more of the social aspects of the American high school stereotype rather than the academic study or career preparation that is associated with Western higher education. Instructors fill a difficult position: they must teach large course loads to support themselves while striving for scarce opportunities for further career advancement and professional development. Administrators constantly seek funding and support to maintain operations, thereby creating practices and outcomes that resemble a business more than an educational institution. A culture of corruption devalues a diploma as well as factors like student attendance, academic performance, and work output. A diploma also offers little in the way of job security; there are few job opportunities within the 
country, so most young people seek expatriate work. Meanwhile public-school teaching positions in the KR are largely open to whomever is available or interested given the substandard working conditions and compensation.

However, higher education in the United States also reflects many of these challenges. Students enter an American university for a variety of purposes, with various levels of preparation, and with largely individual intents. It is possible for university students to do or learn very little and still graduate with a degree. A diploma no longer guarantees gainful employment and may instead confer crippling costs. Like KR universities, US universities increasingly must balance business-like operations with the tenets of academia and principles of higher education. Declining governmental support and increased competition from for-profit higher education institutions have pressured some universities to increase the number of tuition-paying students regardless of the impacts on instructors, students, and the university itself. While corruption and embezzlement are less forthright than bribery, they are just as pervasive and damaging throughout American higher education. Some institutions defraud students with degrees and credentials that are truly worthless in the modern workforce. At others, including the institution this dissertation is being submitted to, university leaders have repeatedly misappropriated funds on a truly brazen scale in clear violation of fundamental ethical and financial standards.

The quality of instruction throughout American higher education, even at prestigious institutions, arguably continues to decline. Under financial strain, universities continue to replace professors with adjunct instructors and determine tenure based on research output and external grant funding. The increasing disillusionment with higher 
education and tenets of anti-intellectualism can be further understood through the experiences of hard-working university students who secure undergraduate and graduate degrees but nevertheless struggle to find gainful employment. The burdens of student loans impact the lives and futures of many individuals throughout the United States; many students took out such loans with expectations that higher education would open doors and lead to fulfilling and sustaining careers only to be derailed by the financial crisis, degrees in theoretical disciplines with little practical value, or oversaturated professional employment marketplaces.

As a doctoral candidate, I have been reflecting on my own experiences and practices in higher education through the lens of the NSSE benchmarks framework. Based on the outcomes of my undergraduate and graduate education, I question the necessity and validity of higher education in the modern global workforce. Although many do not admit it, credentials hinder some employment opportunities and, when coupled with limited experience and significant financial burdens, can make it more difficult to secure a job or eventual career. In addition, substantial career opportunities within academia are declining, bringing the relevance and value of a doctoral education into question. In reflecting critically on my post-secondary education, I recall little in the way of positive student-faculty interactions, supportive campus environments, or enriching educational experiences. On some level, I resonate with the nascent anger and disillusionment I heard from students in the KR toward their own higher education. From my own experiences, the process of American higher education does critically little to foster learner development and even less to support the acquisition of marketable student skills and positive outcomes whatever they may be. 
Research Lessons Learned. The logistical and practical barriers to conducting this dissertation study explain the paucity of research conducted within this region. Travel to and from the research site required three airlines across thirteen time zones at considerable expense, though the time spent aboard aircrafts and in airports proved valuable for time-consuming processes, especially data analysis, formatting, and transcription review. Future researchers in this region should heed the risk of illness in multiple forms and should budget extra time for researchers who are bound to be indisposed temporarily. Similarly, time and schedules are more conceptual ideas than absolutes within the KR. A confirmed meeting time and place would almost always change, with or without notice, and throughout I had to maintain a positive attitude and reschedule at the convenience of the absent participant.

When working with volunteer participants, researchers must accommodate such changes while saving face — something that I highly advise, because constant setbacks from minor inconveniences can become extremely frustrating. From my perspective, the seemingly limitless kindness and accommodation I encountered throughout the research process more than compensated for my difficulties. The question of how I was able to get volunteer participants to essentially work for free over the course of this dissertation was brought up during its defense. Simply put, while cultural practice at the research site does not formally recognize work without pay; the quality of professional relationships that I developed and maintained throughout the region built a common narrative between myself and participants. By admitting that I as a researcher did not have the answers or even the questions, a common objective of understanding the benchmarks and their relationship to higher education at the research site was set. In this way, participants 
were actively participating in the discovery process as we jointly sought to better understand the teaching and learning environment within the program, effective educational practice, and the relative potential for the benchmarks. I removed myself as the expert-specialist and admitted vulnerability in terms of not fully understanding the country or the university and its problems or their solutions. I believe that this admission spurred participants to actively contribute to the research process in hopes of discovering beneficial practices and improving outcomes which are elusive to developed countries.

It is my hope that future researchers in higher education will take the initiative to conduct studies in regions and systems that consistently receive little in the way of academic research. Discovering solutions to complex, unstable, and uncertain educational problems will require that research adequately translates theory to practice and outcomes. Doing so may require that lines of research increasingly blur and that participants take a more active role in fostering greater understanding of problems affecting their communities and developing practical solutions to improve outcomes.

\section{Conclusion}

This study provides an empirical basis for investigating efforts to improve academic quality in a program operating within a post-Soviet higher education institution. Prior quality improvement efforts have had positive intents, but their failure to evince positive and sustainable changes for learners indicate some misguidedness. This study revealed explanatory evidence for these failures by detailing the professional experiences of practitioners in a single university program in the KR using Western evidence-based practices. I used the NSSE benchmarks as a theoretical framework for analyzing the teaching and learning environment within the ethnographic case. However, significant 
financial and practical issues warrant further investigation prior to any potential implementation in this case. These concerns could feasibly negate beneficial returns to students and practitioners alike. In addition, theoretical problems pertaining to the student engagement construct itself warrant further analysis before being employed as a focus of education reform, especially in resource-constricted, high-stakes environments.

I argue for an iterative process for the exploration and identification of effective means for generating transformative change in educational quality and learner outcomes. This continuous process must be dependent on context from research and development through to implementation and practice. Researchers in higher education who wish to develop initiatives to evince positive change in post-secondary education must be able to bridge the gaps between concept and theory, theory and research, research and evidence, evidence and practice, and practice and learner experiences and outcomes. Despite the strength of their basis in theory and evidence, so-called best practices are not panaceas for poorly-defined educational problems, especially when those problems are confounded by change, complexity, and irrationality. Now, more than ever, learning environments need practical solutions to complex problems, and the stakes are higher in developing and transitioning nation states. Without the identification of methods to translate educational theory into effective educational practice, the gap between academic theory and functional reality will only continue to widen.

I conclude this dissertation with a decidedly blunt yet apt quote, "If a problem has no solution, it may not be a problem, but a fact —not to be solved, but to be coped with over time,”(“Shimon Peres on Life, War, and Israel,” 2016). The reality of situations, circumstances, and contexts can mean that even the most grievous of problems will 
remain devoid of effective solutions. The purported boundless optimism of educational reform advocates and visionary organizations fails to consider the unfortunate possibility that efforts and resources committed to solving unsolvable problems might be better spent developing means for impacted communities and individuals to cope. Just as theory can be critiqued as being out of touch with reality, optimistic intents warrant criticism for failing to recognize the nature of intractable and pervasive problems more closely resembling facts. The reality is that problems of inequity and inequality are facts that permeate educational institutions and organizations throughout the world. Acknowledging this unpleasant reality and determining effective methods for those most affected to cope may be in everyone's best interest. 


\section{REFERENCES}

Abashin, S., Abdullaev, K., Abdullaev, R., \& Koichiev, A. (2011). Soviet rule and the delineation of borders in the Ferghana Valley, 1917-1930. Ferghana Valley: The Heart of Central Asia, 94-118.

Abdullaev, K., \& Nazarov, R. (2011). The Ferghana Valley Under Stalin: 1929-1953. In Ferghana Valley: The Heart of Central Asia (pp. 119-139).

Abdullaev, R., Khotamov, N., \& Kenensariev, T. (2011). Colonial Rule and Indigenous Responses, 1860-1917. In Ferghana Valley: The Heart of Central Asia (pp. 6993).

Adams, D. K. (2000). Education and national development: priorities, policies, and planning. Manila, Philippines; Hong Kong: Asian Development Bank; Comparative Education Research Centre, The University of Hong Kong.

ADB. (2015). Assessment of Higher Education: Kyrgyz Republic. Asian Development Bank. Retrieved from https://www.adb.org/documents/assessment-highereducation-kyrgyz-republic

ADB. (2017a). Asian Development Outlook (ADO) 2017: Transcending the MiddleIncome Challenge. Asian Development Bank. http://dx.doi.org/10.22617/FLS178632-3

ADB. (2017b, July 21). Kyrgyz Republic: Economy [Text]. Retrieved August 25, 2017, from https:/www.adb.org/countries/kyrgyz-republic/economy 
Alexander, P. A. (2017). Reflection and reflexivity in practice versus in theory:

Challenges of conceptualization, complexity, and competence. Educational Psychologist, 52(4), 307-314.

Aminov, K., Jensen, V., Juraev, S., Overland, I., Tyan, D., \& Uulu, Y. (2010). Language Use and Language Policy in Central Asia, 29.

Amsler, S. (2008). Higher Education Reform in post-Soviet Kyrgyzstan. Structure and Agency in the Neoliberal University, London: Routledge, 101-28.

Anderson, K., \& Pomfret, R. (2000). Living Standards during Transition to a Market Economy: The Kyrgyz Republic in 1993 and 1996. Journal of Comparative Economics, 28(3), 502-523. https://doi.org/10.1006/jeec.2000.1670

Appleton, J. J., Christenson, S. L., \& Furlong, M. J. (2008). Student engagement with school: Critical conceptual and methodological issues of the construct. Psychology in the Schools, 45(5), 369-386. https://doi.org/10.1002/pits.20303

Argyris, C. (1976). Leadership, Learning, and Changing the Status Quo. Organizational Dynamics, 4(3), 29-43.

Argyris, C., \& Schön, D. A. (1974). Theory in practice: Increasing professional effectiveness. Jossey-Bass.

Astin, A. W. (1984). Student involvement: A developmental theory for higher education. Journal of College Student Personnel, 25(4), 297-308.

Astin, A. W. (1993). What matters in college?: Four critical years revisited (Vol. 1). Jossey-Bass San Francisco.

Avis, G. (1990). The Soviet Higher Education Reform: Proposals and Reactions. Comparative Education, 26(1), 5-12. 
Aypay, A. (2004). Turkish higher education initiatives toward Central Asia. In The challenge of education in Central Asia (pp. 81-96).

Bain, K. (2004). What the Best College Teachers Do.

Baron, P., \& Corbin, L. (2012). Student engagement: rhetoric and reality. Higher Education Research \& Development, 31(6), 759-772. https://doi.org/10.1080/07294360.2012.655711

Bayalieva-Jailobaeva, K. (2017). New Donor Strategies: Implications for NGOs in PostSoviet Kyrgyzstan. VOLUNTAS: International Journal of Voluntary and Nonprofit Organizations, 1-20. https://doi.org/10.1007/s11266-017-9878-5

Berger, R. (2015). Now I see it, now I don't: Researcher's position and reflexivity in qualitative research. Qualitative Research, 15(2), 219-234.

Berkowitz, S. (1997). Analyzing qualitative data. J. Frechtling, L. Sharp, and Westat (Eds.), User-Friendly Handbook for Mixed Method Evaluations, 91.

Beshimov, B., Shozimov, P., \& Bakhadyrov, M. (2011). A new phase in the history of the Ferghana Valley, 1992-2008. Ferghana Valley: The Heart of Central Asia, $205-231$.

Biesta, G. (2007). Why “what works" won't work: Evidence-based practice and the democratic deficit in educational research. Educational Theory, 57(1), 1-22.

Biesta, G. J. (2010). Why ‘what works’ still won’t work: From evidence-based education to value-based education. Studies in Philosophy and Education, 29(5), 491-503. Billups, F. (2014). The quest for rigor in qualitative studies: Strategies for institutional researchers. The NERA Researcher, 1-5. 
Bowen, S. (2005). Engaged Learning: Are We All on the Same Page? Peer Review, 7(2), 4-7.

Brownell, J. E., \& Swaner, L. E. (2009). High-impact practices: Applying the learning outcomes literature to the development of successful campus programs. Peer Review, 11(2), 26.

Brunner, J. J., \& Tillett, A. (2007). Higher Education in Central Asia; the challenges of modernization-an overview. Washington, DC: World Bank. Retrieved from http://200.6.99.248/ bru487cl/files/RevAlldoc2c.pdf

Butler, J. M. (2011). Using standardized tests to assess institution-wide student engagement. Promoting Student Engagement, 1, 258-264.

Byrd, M. W., Lukin, A., Far Eastern Federal University, \& Asia Pacific Center for Security Studies (Honolulu, H. (2012). Education, Economic Growth, and Social Stability: Why the Three are Inseparable. In From APEC 2011 to APEC 2012: American and Russian perspectives on Asia-Pacific security and cooperation (pp. 102-113). Vladivostok; Honolulu: Far Eastern Federal University Press ; AsiaPacific Center for Security Studies.

Chang, H. (2012). Student Engagement and the Quality of Higher Education: A Contextual and Analytical Study of Current Taiwanese Undergraduates. University of California, Davis. Retrieved from http://search.proquest.com/openview/3fee232bce29e883eb9754484613be43/1?pq -origsite $=$ gscholar\&cbl $=18750 \&$ diss $=y$

Chapman, D. W., Weidman, J., Cohen, M., \& Mercer, M. (2005). The search for quality: A five country study of national strategies to improve educational quality in 
Central Asia. International Journal of Educational Development, 25(5), 514-530. https://doi.org/10.1016/j.jedudev.2005.02.003

Chapman, E. (2003). Assessing Student Engagement Rates. ERIC Digest. Retrieved from https://eric.ed.gov/?id=ED482269

Chickering, A. W., \& Gamson, Z. F. (1987). Seven principles for good practice in undergraduate education. AAHE Bulletin, 3, 7.

Chirikov, I. (2015). The Mystery of Russian Students: Poor Learning Experience, High Satisfaction. Higher Education in Russia and Beyond, 1(3), 10-11.

CIRP Freshman Survey - HERI. (n.d.). Retrieved January 8, 2018, from https:/heri.ucla.edu/cirp-freshman-survey/

Coates, H. (2005). The value of student engagement for higher education quality assurance. Quality in Higher Education, 11(1), 25-36. https://doi.org/10.1080/13538320500074915

Coates, H. (2007). A model of online and general campus-based student engagement. Assessment \& Evaluation in Higher Education, 32(2), 121-141. https://doi.org/10.1080/02602930600801878

Coates, H., \& McCormick, A. C. (2014). Engaging University Students: International Insights from System-Wide Studies. Springer.

Country Development Cooperation Strategy. (2017, October 4). Retrieved October 5, 2017, from https://www.usaid.gov/kyrgyz-republic/cdes

Creswell, J. W. (2008). Educational research. Planning, Conducting, and Evaluating Quantitative and Qualitative Research. 
Crouch, M., \& McKenzie, H. (2006). The logic of small samples in interview-based qualitative research. Social Science Information, 45(4), 483-499. https://doi.org/10.1177/0539018406069584

Cummings, S. N., \& Nørgaard, O. (2004). Conceptualising State Capacity: Comparing Kazakhstan and Kyrgyzstan. Political Studies, 52(4), 685-708. https://doi.org/10.1111/j.1467-9248.2004.00503.x

Cussó, R. (2006). Restructuring UNESCO's statistical services—The “sad story" of UNESCO's education statistics: 4 years later. International Journal of Educational Development, 26(5), 532-544.

https://doi.org/10.1016/j.jijedudev.2006.01.001

Dauter, L., \& Olivieri, S. (2017). Education Equality Index. Retrieved from http://www.educationequalityindex.org/wpcontent/uploads/2016/02/GreatSchools_EEI-2017.pdf

Dave Bhavna. (2004). Entitlement through numbers: nationality and language categories in the first post-Soviet census of Kazakhstan*. Nations and Nationalism, 10(4), 439-459. https://doi.org/10.1111/j.1354-5078.2004.00176.x

Davis, M. (2003). Barriers to reflective practice: The changing nature of higher education. Active Learning in Higher Education, 4(3), 243-255.

de la Sablonnière, R., Taylor, D. M., \& Sadykova, N. (2009). Challenges of applying a student-centered approach to learning in the context of education in Kyrgyzstan. International Journal of Educational Development, 29(6), 628-634. https://doi.org/10.1016/j.ijedudev.2009.01.001 
DeYoung, A. (2004). On the current demise of the "Action Plan" for Kyrgyz education reform: A case study. The Challenges of Education in Central Asia, 199-224.

DeYoung, A. J. (2005). Ownership of Education Reforms in the Kyrgyz Republic: kto v dome hozyain? European Educational Research Journal, 4(1), 36-49.

DeYoung, A. J. (2006). Problems and trends in education in Central Asia since 1990: the case of general secondary education in Kyrgyzstan. Central Asian Survey, 25(4), 499-514. https://doi.org/10.1080/02634930701210575

DeYoung, A. J. (2008). Conceptualizing paradoxes of post-socialist education in Kyrgyzstan. Nationalities Papers, 36(4), 641-657.

DeYoung, A. J. (2010). Embracing globalization: university experiences among youth in contemporary Kyrgyzstan. Central Asian Survey, 29(4), 421-434. https://doi.org/10.1080/02634937.2010.535312

DeYoung, A. J. (2011a). Lost in transition: Redefining students and universities in the contemporary Kyrgyz Republic. IAP.

DeYoung, A. J. (2011b). Teaching as a Profession in the Kyrgyz Republic: The Quest for Building/Rebuilding the Knowledge Base. Globalization on the Margins: Education and Postsocialist Transformations in Central Asia. Charlotte, NC: Information Age Publishing.

DeYoung, A. J., \& Santos, C. (2004). Central Asian educational issues and problems. In The challenges of education in Central Asia (pp. 65-80).

Diplomat, R. S., The. (n.d.). The Downside of Foreign Aid in Kyrgyzstan. Retrieved July 9, 2018, from https://hediplomat.com/2017/06/the-downside-of-foreign-aid-inkyrgyzstan/ 
Drummond, T., \& DeYoung, A. J. (2003). Perspectives and problems in education reform in Kyrgyzstan. In The challenges of education in Central Asia (pp. 225-242).

Dubovitskii, V., \& Bababekov, K. (2011). The rise and fall of the Kokand Khanate. Ferghana Valley. The Heart of Central Asia. ME Sharpe, Armonk, 29-68.

Dudwick, N. G., Elizabeth Marc, Alexandre Kuehst, Kathleen. (2003). When Things Fall Apart. The World Bank. https://doi.org/10.1596/0-8213-5067-6

Education, Audiovisual and Culture Executive Agency. (2013). Tempus - Partner countries: Kyrgyzstan. Retrieved from http://eacea.ec.europa.eu/tempus/participating_countries/kyrgystan_en.php

Eklof, B., \& Dneprov, Ė. (1993). Democracy in the Russian school: The reform movement in education since 1984. Westview Press.

Elliott, J., \& Tudge, J. (2007a). The impact of the west on post-Soviet Russian education: change and resistance to change. Comparative Education, 43(1), 93-112. https://doi.org/10.1080/03050060601162420

Elliott, J., \& Tudge, J. (2007b). The impact of the west on post-Soviet Russian education: change and resistance to change. Comparative Education, 43(1), 93-112. https://doi.org/10.1080/03050060601162420

Everett-Heath, T. (2003). Central Asia: Aspects of Transition. Routledge. FEDERATION, R. (n.d.). EQUITY IN EDUCATION THEMATIC REVIEW.

Ferry, N. M., \& Ross-Gordon, J. M. (1998). An Inquiry into Schön's Epistemology of Practice: Exploring Links between Experience and Reflective Practice. Adult Education Quarterly, 48(2), 98-112. https://doi.org/10.1177/074171369804800205 
Fredricks, J. A., Blumenfeld, P. C., \& Paris, A. H. (2004). School Engagement: Potential of the Concept, State of the Evidence. Review of Educational Research, 74(1), 59-109. https://doi.org/10.3102/00346543074001059

Fredricks, J. A., \& Eccles, J. S. (2006a). Extracurricular involvement and adolescent adjustment: Impact of duration, number of activities, and breadth of participation. Applied Developmental Science, 10(3), 132-146.

Fredricks, J. A., \& Eccles, J. S. (2006b). Is extracurricular participation associated with beneficial outcomes? Concurrent and longitudinal relations. Developmental Psychology, 42(4), 698.

Freedberg, L. (2017). In strategy shift, Gates Foundation to spend bulk of education dollars on 'locally driven solutions.' Retrieved March 26, 2018, from https://edsource.org/2017/in-strategy-shift-gates-foundation-to-spend-bulk-ofeducation-dollars-on-locally-driven-solutions/589145

Fresh Start: The Chan Zuckerberg Initiative and the New Era of K-12 Philanthropy. (n.d.). Retrieved July 9, 2018, from https://www.insidephilanthropy.com/home/2016/5/5/fresh-start-the-chanzuckerberg-initiative-and-the-new-era-o.html

Gates, B., \& Gates, M. (2018). 10 tough questions we get asked. Retrieved March 26, 2018, from https://www.gatesnotes.com/2018-Annual-Letter

Geertz, C. (1973). The interpretation of cultures: selected essays. New York: Basic Books.

Ginsburg, M. (2010). Improving educational quality through active-learning pedagogies: A comparison of five case studies (Vol. 1). 
Gita, S.-K. (2016). Teach or Perish: The Stavka System and its Impact on the Quality of Instruction. Вопросы Образования, (2 (eng)). Retrieved from https://cyberleninka.ru/article/n/teach-or-perish-the-stavka-system-and-its-impacton-the-quality-of-instruction

Gonyea, R. M., Kish, K. A., Kuh, G. D., Muthiah, R. N., \& Thomas, A. D. (2003). College Student Experiences Questionnaire: Norms for the Fourth Edition. College Student Experiences Questionnaire Assessment Program (NJ1).

Google. (2017). Kyrgyz Republic Map. Retrieved December 12, 2017, from https://www.google.co.id/maps/place/Kyrgyzstan/@40.8368862,58.0296504,4z/d ata $=! 4 \mathrm{~m} 5 ! 3 \mathrm{~m} 4 ! 1 \mathrm{~s} 0 \times 3897381 \mathrm{dfce} 927 \mathrm{f3}: 0 \times 281058 \mathrm{~b} 74 \mathrm{e} 88 \mathrm{c} 433 ! 8 \mathrm{~m} 2 ! 3 \mathrm{~d} 41.20438 ! 4$ $\mathrm{d} 74.766098 ? \mathrm{hl}=\mathrm{en}$

Grant, C., \& Osanloo, A. (2014). Understanding, selecting, and integrating a theoretical framework in dissertation research: creating the blueprint for your "house." Administrative Issues Journal Education Practice and Research. https://doi.org/10.5929/2014.4.2.9

Guest, G., Bunce, A., \& Johnson, L. (2006). How Many Interviews Are Enough?: An Experiment with Data Saturation and Variability. Field Methods, 18(1), 59-82. https://doi.org/10.1177/1525822X05279903

Hagel, P., Carr, R., \& Devlin, M. (2012). Conceptualising and measuring student engagement through the Australasian Survey of Student Engagement (AUSSE): a critique. Assessment \& Evaluation in Higher Education, 37(4), 475-486. https://doi.org/10.1080/02602938.2010.545870 
Hanushek, E. A., \& Woessmann, L. (2007). The Role of Education Quality for Economic Growth. https://doi.org/10.1596/1813-9450-4122

Hegelund, A. (2005). Objectivity and Subjectivity in the Ethnographic Method. Qualitative Health Research, 15(5), 647-668. https://doi.org/10.1177/1049732304273933

Heyneman, S. P. (1999). The sad story of UNESCO's education statistics. International Journal of Educational Development, 19(1), 65-74. https://doi.org/10.1016/S0738-0593(98)00068-6

Heyneman, S. P. (2009). The Failure of Education for All as Political Strategy. PROSPECTS, 39(1), 5-10. https://doi.org/10.1007/s11125-009-9107-0

Heyneman, S. P. (2010). A comment on the changes in higher education in the former Soviet Union. European Education, 42(1), 76-87.

Heyneman, S. P., \& DeYoung, A. J. (2004). The Challenges of Education in Central Asia. Information Age Pub.

Hill, F. (2001, November 30). The United States and Russia in Central Asia: Uzbekistan, Tajikistan, Afghanistan, Pakistan, and Iran. Retrieved October 31, 2017, from https://www.brookings.edu/on-the-record/the-united-states-and-russia-in-centralasia-uzbekistan-tajikistan-afghanistan-pakistan-and-iran/

Hitchens, C. (2008). Why Orwell Matters. Basic Books.

Hu, S., \& Kuh, G. D. (2002). Being (Dis)Engaged in Educationally Purposeful Activities: The Influences of Student and Institutional Characteristics. Research in Higher Education, 43(5), 555-575. https://doi.org/10.1023/A:1020114231387 
Hufton, N. R., Elliott, J. G., \& Illushin, L. (2002). Educational motivation and engagement: Qualitative accounts from three countries. British Educational Research Journal, 28(2), 265-289.

International Crisis Group. (2016). Kyrgyzstan: State Fragility and Radicalisation (No. 83). Retrieved from https://www.crisisgroup.org/europe-central-asia/centralasia/kyrgyzstan/kyrgyzstan-state-fragility-and-radicalisation

Issakov, K. (2014). Higher education experiences in Kyrgyzstan: case of Osh State University.

Jimerson, S. R., Campos, E., \& Greif, J. L. (2003). Toward an understanding of definitions and measures of school engagement and related terms. The California School Psychologist, 8(1), 7-27.

Johnson, M. K., Crosnoe, R., \& Elder Jr, G. H. (2001). Students' attachment and academic engagement: The role of race and ethnicity. Sociology of Education, 318-340.

Johnson, M. S. (2004). The legacy of Russian and Soviet education and the shaping of ethnic, religious, and national identities in Central Asia. In The Challenges of Education in Central Asia.

Kahu, E. R. (2013). Framing student engagement in higher education. Studies in Higher Education, 38(5), 758-773. https://doi.org/10.1080/03075079.2011.598505

Khazanov, A. M. (1995). After the USSR: Ethnicity, Nationalism and Politics in the Commonwealth of Independent States. Univ of Wisconsin Press.

Kline, G. L. (1957). Soviet education. Columbia University Press. 
Krause, K.-L., \& Coates, H. (2008). Students' engagement in first-year university. Assessment \& Evaluation in Higher Education, 33(5), 493-505. https://doi.org/10.1080/02602930701698892

Kubicek, P. (1997). Regionalism, nationalism and Realpolitik in central Asia. EuropeAsia Studies, 49(4), 637-655. https://doi.org/10.1080/09668139708412464

Kuehnast, K. (2002). Poverty shock: The impact of rapid economic change on the women of the Kyrgyz Republic. When Things Fall Apart: Qualitative Studies of Poverty in the Former Soviet Union, 33-56.

Kuh, G. D. (1991). Involving Colleges: Successful Approaches to Fostering Student Learning and Development outside the Classroom.. ERIC.

Kuh, G. D. (2000). The NSSE 2000 report: National benchmarks for effective educational practice. Bloomington, IN: Center for Postsecondary Research and Planning.

Kuh, G. D. (2003). What We're Learning About Student Engagement from NSSE. Change, 35(2), 24.

Kuh, G. D. (2006). Making students matter. Fixing the Fragmented University: Decentralization with Direction, 235-264.

Kuh, G. D. (2009a). The National Survey of Student Engagement: Conceptual and Empirical Foundations. New Directions for Institutional Research, 2009(141), 520. https://doi.org/10.1002/ir.283

Kuh, G. D. (2009b). What student affairs professionals need to know about student engagement. Journal of College Student Development, 50(6), 683-706. 
Kuh, G. D., Kinzie, J., Buckley, J. A., Bridges, B. K., \& Hayek, J. C. (2011). Piecing together the student success puzzle: research, propositions, and recommendations: ASHE Higher Education Report (Vol. 116). John Wiley \& Sons. Retrieved from http://books.google.com/books?hl=en\&lr=\&id=E2Y15q5bpCoC\&oi=fnd\&pg=PR $7 \& \mathrm{dq}=\% 22$ the + conditions + that + foster + student + success + in + college + has + never + been + more + important. $\% 22+\% 22 \mathrm{in}+$ the + economic + ladder $+($ Bowen $+1978 \% 3 \mathrm{~B}+$ Bowen + and + Bok $+1998 \% 3 B+$ Boyer + and + Hechinger $+1981 \% 3 B \% 22+\&$ ots $=$ Zxz RkfwmLP\&sig=JkUxZI5NGmWgYBqhMfBLRVmeWbw

Kuh, G. D., Kinzie, J. L., Buckley, J. A., Bridges, B. K., \& Hayek, J. C. (2006). What matters to student success: A review of the literature (Vol. 8). National Postsecondary Education Cooperative Washington, DC.

Kuh, G. D., Schuh, J. H., \& Whitt, E. J. (1991). Some good news about campus life. Change, 23(5), 48.

Kuh, George D. (2005). NSSE-National Survey of Student Engagement: Conceptual Framework and Overview of Psychometric Properties. In Paper presented at the. Retrieved from http://nsse.indiana.edu/html/psychometric_framework_2002.cfm

Lamborn, S., Newmann, F., \& Wehlage, G. (1992). The significance and sources of student engagement. Student Engagement and Achievement in American Secondary Schools, 11-39.

LaNasa, S. M., Cabrera, A. F., \& Trangsrud, H. (2009). The Construct Validity of Student Engagement: A Confirmatory Factor Analysis Approach. Research in Higher Education, 50(4), 315-332. https://doi.org/10.1007/s11162-009-9123-1 
Landau, J. M., \& Kellner-Heinkele, B. (2011). Language Politics in Contemporary Central Asia: National and Ethnic Identity and the Soviet Legacy. London, UNITED STATES: I.B.Tauris. Retrieved from http://ebookcentral.proquest.com/lib/louisville/detail.action?docID=903303

Lawson, M. A., \& Lawson, H. A. (2013). New Conceptual Frameworks for Student Engagement Research, Policy, and Practice. Review of Educational Research, 83(3), 432-479. https://doi.org/10.3102/0034654313480891

Leach, L., \& Zepke, N. (2011). Engaging students in learning: a review of a conceptual organiser. Higher Education Research \& Development, 30(2), 193-204. https://doi.org/10.1080/07294360.2010.509761

Lee, J.-W. (2007). Economic growth and human development in the Republic of Korea, 1945-1992. Reconstruction, 1945, 61.

Lerer, N., \& Talley, K. (2010). National Survey of Student Engagement's (NSSE) benchmarks - one size fits all? On the Horizon, 18(4), 355-363. https://doi.org/10.1108/10748121011082653

Levy, S. E., \& Hawkins, D. E. (2009). Peace through tourism: Commerce based principles and practices. Journal of Business Ethics, 89, 569-585.

Lincoln, Y. S., \& Guba, E. G. (1985). Naturalistic inquiry. Beverly Hills, Calif.: Sage Publications.

Lutz, M. E., \& Culver, S. (2010). The National Survey of Student Engagement: A university-level analysis. Tertiary Education and Management, 16(1), 35-44. 
Macfarlane, B., \& Tomlinson, M. (2017). Critical and Alternative Perspectives on Student Engagement. Higher Education Policy, 30(1), 1-4. https://doi.org/10.1057/s41307-016-0026-4

Madaminzhanova, Z., \& Mukhtarov, I. (2011). Cultural Life in the Ferghana Valley Under Khrushchev and Brezhnev. Ferghana Valley: The Heart of Central Asia.

Maguire, R., Egan, A., Hyland, P., \& Maguire, P. (2017). Engaging students emotionally: the role of emotional intelligence in predicting cognitive and affective engagement in higher education. Higher Education Research \& Development, 36(2), 343-357. https://doi.org/10.1080/07294360.2016.1185396

Mandernach, B. J. (2015). Assessment of Student Engagement in Higher Education: A Synthesis of Literature and Assessment Tools. International Journal of Learning, Teaching and Educational Research, 12(2). Retrieved from https://www.ijlter.org/index.php/ijlter/article/view/367

Mandernach, B. J., Donnelli-Sallee, E., \& Dailey-Hebert, A. (2011). Assessing course student engagement. Promoting Student Engagement, 1. Retrieved from http://www.academia.edu/download/24927796/54._course_engagement.pdf Markowitz, F. (2000). Coming of age in post-Soviet Russia. University of Illinois Press Urbana.

Martin, A. J., Ginns, P., \& Papworth, B. (2017). Motivation and engagement: Same or different? Does it matter? Learning and Individual Differences, 55(Supplement C), 150-162. https://doi.org/10.1016/j.lindif.2017.03.013

Martin, A. J., Papworth, B., Ginns, P., Malmberg, L.-E., Collie, R. J., \& Calvo, R. A. (2015). Real-time motivation and engagement during a month at school: Every 
moment of every day for every student matters. Learning and Individual Differences, 38, 26-35. https://doi.org/10.1016/j.lindif.2015.01.014

McCormick, A. C., Kinzie, J., \& Gonyea, R. M. (2013). Student Engagement: Bridging Research and Practice to Improve the Quality of Undergraduate Education. In Higher Education: Handbook of Theory and Research (pp. 47-92). Springer, Dordrecht. https://doi.org/10.1007/978-94-007-5836-0_2

McCormick, A. C., \& McClenney, K. (2012). Will These Trees Ever Bear Fruit?: A Response to the Special Issue on Student Engagement. The Review of Higher Education, 35(2), 307-333. https://doi.org/10.1353/rhe.2012.0010

McGlinchey, E. (2011). Exploring regime instability and ethnic violence in Kyrgyzstan. Asia Policy, 12(1), 79-98.

McLean, G. N., Karimov, M., \& Asankanov, A. (2004). The role of human resource development in improving K-12 educational leadership in Kyrgyzstan. KEDI Journal of Educational Policy, 1(1).

Megoran, N. (2013). Shared Space, Divided Space: Narrating Ethnic Histories of Osh. Environment and Planning A, 45(4), 892-907. https://doi.org/10.1068/a44505

Merrill, M., Yakubova, S., \& Turlanbekova, Z. (2015). Internationalizing Quality Assessment in Central Asia. International Higher Education, O(64). https://doi.org/10.6017/ihe.2011.64.8561

Mertaugh, M. (2004). Education in Central Asia, with particular reference to the Kyrgyz Republic. In The Challenge of Education in Central Asia. Greenwich Connecticut, Information Age Publishing. Retrieved from 
http://siteresources.worldbank.org/INTECONEVAL/Resources/EducInCentralAsi a_Kyrguz_Rep.pdf

Miles, M. B., \& Huberman, A. M. (1994). Qualitative data analysis: An expanded sourcebook. sage.

Miles, M. B., Huberman, A. M., \& Saldana, J. (2014). Qualitative Data Analysis: A Methods Sourcebook (Third). Retrieved from https://us.sagepub.com/enus/nam/qualitative-data-analysis/book239534

National Council for Sustainable Development of the Kyrgyz Republic. (2012a). Education Sector Plan. Kyrgyz Republic. Retrieved from http://www.globalpartnership.org/content/education-development-strategykyrgyz-republic-2012-2020

National Council for Sustainable Development of the Kyrgyz Republic. (2012b). National Sustainable Development Strategy for the Kyrgyz Republic. Retrieved from http://www.unpage.org/files/public/kyrgyz_national_sustainable_development_strategy.pdf Nazarov, R., \& Shozimov, P. (2011). The Ferghana Valley in the Eras of Khruschev and Brezhnev. In Ferghana Valley: The Heart of Central Asia (pp. 140-163).

Nazarov, U. (2016). Kyrgyzstan launches education reform to fight extremism. Retrieved September 26, 2017, from http://central.asianews.com/en_GB/articles/cnmi_ca/features/2016/09/21/feature-01

Nessipbayeva, O., \& Dalayeva, T. (2013). Developmental Perspectives of Higher Education in the Post-Soviet Countries (for the Cases of Kazakhstan, Uzbekistan, 
Kyrgyzstan, Tajikistan and Turkmenistan). Procedia - Social and Behavioral Sciences, 89, 391-396. https://doi.org/10.1016/j.sbspro.2013.08.865

Niyozov, S. (2006). The Effects of the Collapse of the USSR on Teachers' Lives and Work in Tajikistan. In The challenges of education in Central Asia (p. 37).

Niyozov, S., \& Shamatov, D. (2006). Trading or teaching: Dilemmas of everyday life economy in Central Asia. Inner Asia, 8(2), 229-62.

NSSE About NSSE. (n.d.). Retrieved September 13, 2018, from http://nsse.indiana.edu/html/about.cfm

NSSE Benchmarks. (2000).

NSSE Engagement Indicators and High-Impact Practices. (2015). Retrieved from http://nsse.indiana.edu/pdf/EIs_and_HIPs_2015.pdf

NSSE Origins and Potential. (n.d.). Retrieved January 8, 2018, from http://nsse.indiana.edu/html/origins.cfm

Olivas, M. A. (2011). If You Build It, They Will Assess It (or, An Open Letter to George Kuh, with Love and Respect). The Review of Higher Education, 35(1), 1-15. https://doi.org/10.1353/rhe.2011.0032

Omelicheva, M. Y. (2015). Democracy in Central Asia : competing perspectives and alternative strategies.

Organisation for Economic Co-operation and Development. (2010). The High Cost of Low Educational Performance The Long-run Economic Impact of Improving PISA Outcomes. Paris: OECD Publishing.

Organization for Security and Co-operation in Europe. (2003). Current Education Issues in Central Asia. Retrieved from http://www.osce.org/cpc/39626?download=true 
Orusbaev, A., Mustajoki, A., \& Protassova, E. (2008). Multilingualism, Russian Language and Education in Kyrgyzstan. International Journal of Bilingual Education and Bilingualism, 11(3-4), 476-500.

https://doi.org/10.1080/13670050802148806

Osh State University. (2017). In Wikipedia. Retrieved from

https://en.wikipedia.org/w/index.php?title=Osh_State_University\&oldid=815324 831

Osipian, A. L. (2009). Corruption hierarchies in higher education in the former Soviet Bloc. International Journal of Educational Development, 29(3), 321-330. https://doi.org/10.1016/j.ijedudev.2008.08.006

Pace, C. R. (1982). Achievement and the Quality of Student Effort.

Pace, C. R. (1984). Measuring the Quality of College Student Experiences. An Account of the Development and Use of the College Student Experiences Questionnaire.

Pascarella, E. T., Seifert, T. A., \& Blaich, C. (2010). How Effective are the NSSE Benchmarks in Predicting Important Educational Outcomes? Change: The Magazine of Higher Learning, 42(1), 16-22. https://doi.org/10.1080/00091380903449060

Pascarella, E. T., \& Terenzini, P. T. (2005). How college affects students: A third decade of research (Vol. 2). San Francisco: Jossey-Bass.

Pfeffer, F. T. (2015). Equality and quality in education. A comparative study of 19 countries. Social Science Research, 51, 350-368. https://doi.org/10.1016/j.ssresearch.2014.09.004 
Pike, G. (2013). NSSE Benchmarks and Institutional Outcomes: A Note on the Importance of Considering the Intended Uses of a Measure in Validity Studies. Research in Higher Education, 54(2), 149-170. https://doi.org/10.1007/s11162012-9279-y

Pilot Participant. (2017).

Polit, D. F., Tatano Beck, C., \& Timmins, F. (2013). Nursing Research Generating and Assessing Evidence for Nursing Practice. Nurse Education in Practice, 13(6), e29-e29.

Porter, S. R. (2011). Do college student surveys have any validity? The Review of Higher Education, 35(1), 45-76.

Porter, S. R., Rumann, C., \& Pontius, J. (2011). The validity of student engagement survey questions: can we accurately measure academic challenge? New Directions for Institutional Research, 2011(150), 87-98.

Reeves, M. (2004). Cultivating citizens of a 'new type': The politics and practice of educational reform at the American University in Kyrgyzstan. In The challenges of education in Central Asia (pp. 365-85).

Robinson, J. P., \& Winthrop, R. (2016). Millions Learning: Scaling up Quality Education in Developing Countries. Center for Universal Education at The Brookings Institution. Retrieved from https://eric.ed.gov/?id=ED568939

Routray, B. P. (2017). Islamic State in Central Asia: Threat as an alibi to repression. Retrieved from http://mantraya.org/wp-content/uploads/2017/06/Islamic-State-inCentral-Asia_BibhuRoutray_Mantraya.pdf 
Ruget, V., \& Usmanalieva, B. (2007). The impact of state weakness on citizenship a case study of Kyrgyzstan. Communist and Post-Communist Studies, 40(4), 441-458. https://doi.org/10.1016/j.postcomstud.2007.10.002

Ryan, A., Tilbury, D., Corcoran, P. B., Abe, O., \& Nomura, K. (2010). Sustainability in higher education in the Asia-Pacific: developments, challenges, and prospects. International Journal of Sustainability in Higher Education, 11(2), 106-119. https://doi.org/10.1108/14676371011031838

Sahlberg, P. (2006). Education Reform for Raising Economic Competitiveness. Journal of Educational Change, 7(4), 259-287. https://doi.org/10.1007/s10833-005-48846

Saidov, A., Anarbaev, A., \& Goriyacheva, V. (2011). The Ferghana Valley: the precolonial legacy. In The Ferghana valley: the heart of Central Asia. Armonk, NY: ME Sharpe. Pp3-28.

Sainazarov, K., \& Shamatov, D. (2010). The impact of standardized testing on education quality in Kyrgyzstan: The case of the Program for International Student Assessment (PISA) 2006. In The Impact of International Achievement Studies on National Education Policymaking (Vol. 13, pp. 145-179). Emerald Group Publishing Limited. https://doi.org/10.1108/S1479-3679(2010)0000013009

Saldaña, J. (2009). The Coding Manual for Qualitative Researchers. SAGE Publications, Incorporated.

Saldaña, J. (2015). The coding manual for qualitative researchers. Sage.

Schön, D. A. (1983). The Reflective Practitioner: How Professionals Think In Action. Basic Books. 
Seale, J. (2010). Doing student voice work in higher education: an exploration of the value of participatory methods. British Educational Research Journal, 36(6), 995-1015. https://doi.org/10.1080/01411920903342038

Semere, S. (2015). Making A Lasting Impact: The Quality Learning Project Sustainability Framework (A Case Study). Retrieved from http://www.creativeassociatesinternational.com/wpcontent/uploads/2017/05/QLP_Casestudy.pdf

Shamatov, D. A. (2006). Beginning teachers' professional socialization in post-Soviet Kyrgyzstan: The challenges and coping strategies.

Shapiro. (2017). Completing College: A National View of Student Completion Rates Fall 2011 Cohort (Signature Report No. 14). Retrieved from https://nscresearchcenter.org/wp-content/uploads/SignatureReport14_Final.pdf

Shimon Peres on Life, War, and Israel: 10 Best Quotes. (2016, September 28). Haaretz. Retrieved from https://www.haaretz.com/israel-news/shimon-peres-on-life-warand-israel-10-best-quotes-1.5443972

Shozimov, P., Beshimov, B., \& Yunusova, K. (2011). The Ferghana Valley during perestroika, 1985-1991. Ferghana Valley: The Heart of Central Asia, 178-204.

Shulman, L. S. (2002). Making Differences. Change, 34(6), 36.

Sia, E. (2014). Student motivation, intercultural competence and transnational higher education: Uzbekistan, a case study. Journal of the Scholarship of Teaching and Learning, 15(1), 57. https://doi.org/10.14434/josotl.v15i1.13000 
Silova, I. (2009). The Crisis of the Post-Soviet Teaching Profession in the Caucasus and Central Asia. Research in Comparative and International Education, 4(4), 366383. https://doi.org/10.2304/rcie.2009.4.4.366

Silova, I. (2011). Globalization on the margins: Education and post-socialist transformations in Central Asia. IAP.

Simon, M. K., \& Goes, J. (2013). Assumption, limitations, delimitations, and scope of the study. Dissertation and scholarly research: Recipes for success.

Smolentseva, A. (2012). Access to higher education in the post-Soviet States: Between Soviet legacy and global challenges. In Paper commissioned and presented at Salzburg Global Seminar, Session (Vol. 495, pp. 2-7). Retrieved from https://www.researchgate.net/profile/Anna_Smolentseva/publication/268035227_ Access_to_Higher_Education_in_the_PostSoviet_States_Between_Soviet_Legacy_and_Global_Challenges/links/545fb6840 cf27487b450a9db.pdf

Smolentseva, A. (2017). Where Soviet and neoliberal discourses meet: the transformation of the purposes of higher education in Soviet and post-Soviet Russia. Higher Education, 74(6), 1091-1108. https://doi.org/10.1007/s10734-017-0111-7

Starr, S. F. (2011). Ferghana valley: The heart of central asia. ME Sharpe.

Tabulawa, R. (2003). International Aid Agencies, Learner-Centred Pedagogy and Political Democratisation: A Critique. Comparative Education, 39(1), 7-26.

Tate, S., Shamatov, D., \& Weeks-Earp, E. (2011). Kyrgyzstan and Tajikistan Quality Learning Project Program Assessment and Recommendations. Retrieved from http://pdf.usaid.gov/pdf_docs/pdacu716.pdf 
Tchoroev, T. (2002). Historiography of Post-Soviet Kyrgyzstan. International Journal of Middle East Studies, 34(2), 351-374. https://doi.org/10.1017/S0020743802002118

The Sentinel Project for Genocide Prevention. (2013). The Risk of Genocide in Kyrgyzstan 2013. Retrieved from https://thesentinelproject.org/kyrgyzstan/

Thelin, J. R. (2007). Expectations and reality in American higher education. Thought \& Action, 59.

Tinto, V. (2006). Research and practice of student retention: What next? Journal of College Student Retention: Research, Theory \& Practice, 8(1), 1-19.

Tinto, V. (2010). From Theory to Action: Exploring the Institutional Conditions for Student Retention. In J. C. Smart (Ed.), Higher Education: Handbook of Theory and Research (Vol. 25, pp. 51-89). Dordrecht: Springer Netherlands. https://doi.org/10.1007/978-90-481-8598-6_2

Tinto, V. (2012). Completing College: Rethinking Institutional Action. University of Chicago Press.

Trowler, P., \& Trowler, V. (2010a). Student engagement evidence summary. Retrieved from http://eprints.lancs.ac.uk/61680/1/Deliverable_2._Evidence_Summary._Nov_201 $0 . p d f$

Trowler, P., \& Trowler, V. (2010b). Student Engagement Literature Review. The Higher Education Academy, 11, 1-15.

Tyler, Ralph W. (2013). Basic principles of curriculum and instruction. University of Chicago press. 
Tyler, Ralph Winfred. (1932). Service studies in higher education. Ohio state university.

UNICEF CIEES. (2011). Teachers: A Regional Study on Recruitment, Development and Salaries of Teachers.

United Nations Educational, Scientific and Cultural Organization. (2011). The hidden crisis: armed conflict and education (p. 416). Paris.

U.S. Agency for International Development. (2014). COUNTRY DEVELOPMENT COOPERATION STRATEGY, 64.

Validity Evidence - Research - The College Board. (n.d.). Retrieved January 8, 2018, from https://research.collegeboard.org/services/aces/validity/handbook/evidence

Wefald, A. J., \& Downey, R. G. (2009). Construct Dimensionality of Engagement and its Relation With Satisfaction. The Journal of Psychology, 143(1), 91-112. https://doi.org/10.3200/JRLP.143.1.91-112

Wolf-Wendel, L., Ward, K., \& Kinzie, J. (2009). A Tangled Web of Terms: The Overlap and Unique Contribution of Involvement, Engagement, and Integration to Understanding College Student Success. Journal of College Student Development, 50(4), 407-428. https://doi.org/10.1353/csd.0.0077

Yin, R. K. (2017). Case study research and applications: Design and methods. SAGE Publications.

Zepke, N. (2014). Student engagement research in higher education: questioning an academic orthodoxy. Teaching in Higher Education, 19(6), 697-708. https://doi.org/10.1080/13562517.2014.901956 
Zepke, N., \& Leach, L. (2010). Improving student engagement: Ten proposals for action. Active Learning in Higher Education, 11(3), 167-177. https://doi.org/10.1177/1469787410379680 


\section{APPENDICES}

$\begin{array}{ll}\text { APPENDIX } & \text { PAGE }\end{array}$

A. Institutional Review Board Protocol....................................... 216

B. First Interview Protocol Item Rationale...................................... 220

C. Second Interview Protocol Item Rationale.................................. 222

D. Semi-Structured Interview Protocols...................................... 224

E. NSSE Benchmarks .................................................. 227

F. Classroom Observation Instrument...................................... 228

G. Preamble........................................................... 231 


\section{Appendix A: Institutional Review Board (IRB) Protocol}

\section{Exploring Western Evidence-Based Practice in Post-Soviet Higher Education}

Tracking Number 18.0344

Principal Investigator Jacob P. Gross

Co-Investigator J. L. Mettille

\section{Project Summary}

This protocol provides the agenda for the study, practical considerations for study execution, and an overview for institutional review. This dissertation explores the perceptions of Western practices associated with the student engagement construct among post-Soviet higher education practitioners. It seeks to develop a greater understanding of professional higher education experiences in the Kyrgyz Republic (KR) relative to benchmarks of effective educational practice developed in the United States. By exploring practitioner experiences, practices, and perceptions; higher education reform efforts aimed at changing post-secondary education in the KR to improve quality can be further nuanced to reflect the realities of education for faculty and students in this nation.

\section{Background and Problem Statement}

Since declaring independence over thirty years ago, the KR has remained in an operational survival mode as it transitions to democratic governance and a market economy (International Crisis Group, 2016). Education has also continued to transition, and while access to education especially at the post-secondary level has been maintained or expanded, educational quality has declined significantly (Gita, 2016; Smolentseva, 2012, 2017). International governments and donor agencies have stepped in to support the KR in building nation-state capabilities and capacities; however, education in the country has resisted change and sustainable projects have proven to be short-lived (Amsler, 2008; Bayalieva-Jailobaeva, 2017; A. J. DeYoung, 2006; Drummond \& DeYoung, 2003). A gap in the literature exists concerning practitioners in a post-Soviet context reflecting on their professional experiences and practices relative to proposed reform efforts. This study addresses that gap by exploring higher education practitioner perceptions and professional experiences relative to Western practices commonly used in reform and training initiatives.

\section{Objectives and Justification}

By exploring practitioners' professional experiences and perceptions of Western benchmarked practices, evidence obtained could explain reform failure to impact praxis and persistently low educational quality. In addition, the benchmarked practices in this study are from the National Survey of Student Engagement (NSSE) and developed as 
indicators of the student engagement construct (Kuh, 2000, 2009). This construct remains conceptually divisive with considerable debate ongoing in higher education research (Baron \& Corbin, 2012; Coates \& McCormick, 2014; Zepke, 2014). This study lends additional perspective to that dialogue by examining it in a developing post-Soviet higher education context. Evidence obtained could inform future higher education reform efforts pertaining to practice and improve their efficacy. From a much larger perspective, improving the quality of education in the $\mathrm{KR}$ is important to its development and stability (Organisation for Economic Co-operation and Development, 2010), the maintenance of which is of international and regional interest (Hill, 2001; McGlinchey, 2011). The United States also struggles with educational quality improvement on a much larger scale, and evidence obtained may impact those efforts (Freedberg, 2017; Gates \& Gates, 2018). The central research question is, "How do practitioners reflect on their professional experiences and benchmarks of effective educational practice?"

\section{Study Design and Methods}

This analysis employs ethnographic case study design and qualitative field research methods as defined by Creswell (2008). Higher education practitioner participants are the units of analysis and the study scope is restricted to practices within a single foreign language teacher preparation program at a regional university in the south of the KR. The host university lacks an IRB but has granted the co-investigator access to conduct interviews and class observations, in exchange for the co-investigator providing professional development training for faculty and language assessment instruction for students. Approval for this study was secured as the co-investigator previously completed a teaching fellowship in the region. Two twenty-person cohorts of practitioners and students have agreed to participate on a completely voluntary basis to support the completion of this dissertation. Those practitioners and students willing to participate will receive an invitation to interview via preamble (see Appendix G).

\section{Participant Recruitment Methods}

The co-investigator completed a teaching fellowship in Central Asia one-year prior and has maintained a professional association with many individuals, organizations, and universities throughout the region. In discussing the dissertation topic with colleagues in the KR, many faculty members and students volunteered to participate out of professional courtesy. This resulted in the generation of both cohorts within the foreign language teacher preparation program at the host institution research site to which the co-investigator was granted access. With twenty faculty members and twenty students, the sample size is in line with qualitative interview research standards (Crouch \& McKenzie, 2006; Guest et al., 2006).

\section{Human Participant Protections}

Confidentiality, non-disclosure, and security define the human subject protections being incorporated into this protocol. The specific research site including the host university and program is obfuscated throughout at the request of administrators. 
Confidentiality is affirmed as not all cohort volunteers, or their names, are known to the co-investigator despite previous professional exposure. All interviews are recorded on an encrypted mobile phone before being transcribed on an encrypted laptop with contents being uploaded to a password-protected cloud database as a safeguard against data loss. As names of participants are not collected and identifiers are restricted to participant order numbers and transcribed content, their disclosure is restricted. Interviews will be replicated among all volunteer participants and interviews will take place off campus. Contact information of participants obtained for organization and scheduling purposes will be restricted to the encrypted phone and laptop and deleted upon study completion. The security of all participants and the co-investigator will be supported by observing universal safety precautions and U.S. Embassy security guidelines.

\section{Informed Consent and Waiver Process}

A preamble was developed as a means of providing informed consent without obtaining participant signatures (see Appendix G). Some of the volunteer-participants included in this study previously expressed hesitance about providing their names and signatures on other documentation. To respect their concerns and uphold their confidentiality the preamble and verbal confirmation of informed consent procedures were used in place of signed consent documents. The study also represented no more than minimal risk of harm to participants and involves procedures such as interviews and observations which regularly take place without informed consent for non-research purposes.

\section{Research Procedures}

Faculty participants will have a first interview using the developed semistructured interview protocols (see Appendix D), one of their classes will be directly observed with observations noted using a purposefully designed instrument (see Appendix F), and a second round of interviews will be conducted. Final-year student volunteers with sufficient English language abilities over the age of 18 will be invited to interview via the same procedure and protocol. All interviews were recorded and saved to an encrypted phone before being transcribed on a secure laptop along with observation results prior to data analysis.

\section{Minimizing Risks}

The use of a preamble, the inclusion of volunteer participants, and conducting interviews off-campus minimize foreseeable risks at the outset of the study. The coinvestigator will remain responsive to participant concerns throughout the data collection process and will contact the principal investigator and IRB should any changes need to be made on a risk mitigation basis.

\section{Plan for Analysis of Results}


Whereas study design and data collection focused on Creswell's (2008) interpretation of qualitative ethnographic case study; data analysis centered around the analytical strategies of data condensation, data display, and drawing and verifying conclusions (Miles et al., 2014). In addition, the data condensation stage of analysis employs specific coding procedures (Saldaña, 2009).

\section{Research Materials, Records, and Privacy}

All adapted research materials are properly cited with reference to their original developers, and in the case of the classroom observation instrument approval was obtained from the original developer prior to adaptation (S. Harris, personal communication, April 8, 2018). All interviews with participant responses will be deleted upon final dissertation acceptance in order to further minimize any potential threats to participant privacy.

\section{Timetable}

This study is being submitted under expedited review as a category 7 proposal, time frames for travel to the research site are dependent upon IRB approval and restricted by academic calendars and limited financial resources.

\begin{tabular}{ll}
\hline Target Date & Activity \\
$4 / 09 / 2018$ & IRB Research Protocol Submission \\
4/23/2018 & IRB Research Protocol Approval \\
4/30/2018 & Travel to Research Site and Begin Data Collection and Data Analysis \\
$5 / 18 / 2018$ & Return from Research Site and Complete Data Analysis \\
$5 / 25 / 2018$ & Chapters Four and Five to Dissertation Chair \\
$6 / 01 / 2018$ & Chapters Four and Five to Dissertation Committee \\
$6 / 11 / 2018$ & Final Dissertation Defense
\end{tabular}




\begin{tabular}{|c|c|c|c|}
\hline \multicolumn{4}{|c|}{ Appendix B: First Interview Protocol Item Rationale } \\
\hline \multicolumn{4}{|c|}{$\begin{array}{c}\text { Research Question: How do practitioners reflect on their professional experiences } \\
\text { and benchmarks of effective educational practice? }\end{array}$} \\
\hline $\begin{array}{c}\text { Sub- } \\
\text { Questions }\end{array}$ & $\begin{array}{l}\text { Interview } \\
\text { Questions }\end{array}$ & Interview Question Probes & $\begin{array}{c}\text { NSSE } \\
\text { Benchmarks }\end{array}$ \\
\hline $\begin{array}{c}\text { How are } \\
\text { practitioner } \\
\text { higher } \\
\text { education } \\
\text { experiences } \\
\text { described? }\end{array}$ & $\begin{array}{c}\text { How would you } \\
\text { describe your } \\
\text { time in } \\
\text { university? }\end{array}$ & $\begin{array}{l}\text { What was your goal when you enrolled? } \\
\text { Why did you attend the program and university that you did? } \\
\text { How challenging or academically rigorous was your program? } \\
\text { How would you describe the best and worst teachers you had at } \\
\text { university? } \\
\text { How did you interact with your teachers? } \\
\text { How would you describe the students in your program? } \\
\text { How would you describe the best and worst students in your program? } \\
\text { How did you interact with other students inside and outside of class? } \\
\text { Were there opportunities to apply what you were learning outside of } \\
\text { class? } \\
\text { What were your assignments, classes, and examinations like? } \\
\text { What did you learn over the course of your studies? } \\
\text { How did you learn as an undergraduate student? } \\
\text { What problems did you encounter as a student? } \\
\text { How were those problems resolved? } \\
\text { How did the campus environment support you and your learning? } \\
\text { What was a critical or defining moment in your undergraduate } \\
\text { experience? }\end{array}$ & $\begin{array}{c}\text { Level of } \\
\text { Academic } \\
\text { Challenge } \\
\text { Student-Faculty } \\
\text { Interaction } \\
\text { Enriching } \\
\text { Educational } \\
\text { Experiences } \\
\text { Active and } \\
\text { Collaborative } \\
\text { Learning } \\
\text { Supportive } \\
\text { Campus } \\
\text { Environment }\end{array}$ \\
\hline $\begin{array}{c}\text { How are } \\
\text { effective } \\
\text { educational } \\
\text { practices } \\
\text { among } \\
\text { faculty and } \\
\text { students } \\
\text { defined? }\end{array}$ & $\begin{array}{l}\text { How would you } \\
\text { describe your } \\
\text { professional } \\
\text { experience as a } \\
\text { faculty member } \\
\text { and how would } \\
\text { you describe } \\
\text { your students' } \\
\text { experiences? }\end{array}$ & $\begin{array}{l}\text { How would you describe yourself as a teacher? } \\
\text { What standards exist for teachers and for students? } \\
\text { What expectations do you have for your students? } \\
\text { How do students interact with one another inside and outside of class? } \\
\text { What are your assignments, classes, and examinations like? } \\
\text { What teaching methods do you find help students learn best? } \\
\text { How is technology incorporated into teaching and learning? } \\
\text { What problems do you encounter as a teacher? } \\
\text { How do students interact with you as a teacher? }\end{array}$ & $\begin{array}{l}\text { Active and } \\
\text { Collaborative } \\
\text { Learning }\end{array}$ \\
\hline
\end{tabular}




\begin{tabular}{|l|l|c|}
\hline \multirow{n}{*}{} & $\begin{array}{l}\text { How much time do you have to dedicate to research? } \\
\text { How would you describe the best and worst students you have had as } \\
\text { a teacher? }\end{array}$ & \\
& What do students do in between and after classes? & \\
& How is student feedback on learning and progress measured? & Enriching \\
& What do your students struggle to learn? & Educational \\
& How challenging would you say this program is today? & Experiences \\
& How would you describe the campus environment today? & \\
& How does the university encourage and support students and teachers? & Level of \\
& How would you describe Kyrgyz higher education? & Academic \\
& What do you think higher education is like in the United States? & Challenge \\
& How well would you expect US university practices to work here? & \\
& & \\
& & Supportive \\
& & Campus \\
& & Environment \\
\hline
\end{tabular}




\begin{tabular}{|c|c|c|c|}
\hline & Apper & dix C: Second Interview Protocol Item Rationale & \\
\hline & $\begin{array}{r}\text { Research Question: } \\
\text { and }\end{array}$ & $\begin{array}{l}\text { How do practitioners reflect on their professional experiences } \\
\text { benchmarks of effective educational practice? }\end{array}$ & \\
\hline Sub-Question & $\begin{array}{l}\text { Interview } \\
\text { Questions }\end{array}$ & Interview Question Probes & $\begin{array}{c}\text { NSSE } \\
\text { Benchmarks }\end{array}$ \\
\hline & $\begin{array}{l}\text { How challenging is } \\
\text { this academic } \\
\text { program? }\end{array}$ & $\begin{array}{l}\text { How much academic effort is required to succeed in the program? } \\
\text { What are the expectations for students? } \\
\text { What must students do to successfully complete this program? } \\
\text { How much time do students spend outside of class preparing, } \\
\text { studying, and working on course material? } \\
\text { How many papers or reports are required for each class? } \\
\text { How does this program emphasize spending time studying and } \\
\text { completing academic work? }\end{array}$ & $\begin{array}{l}\text { Level of } \\
\text { Academic } \\
\text { Challenge }\end{array}$ \\
\hline $\begin{array}{c}\text { How are the } \\
\text { NSSE } \\
\text { benchmarks and }\end{array}$ & $\begin{array}{l}\text { To what extent is } \\
\text { learning active and } \\
\text { collaborative? }\end{array}$ & $\begin{array}{l}\text { How involved are students with their learning? } \\
\text { How is problem-solving emphasized? } \\
\text { How comfortable are students asking questions during class? } \\
\text { How are class discussions conducted? } \\
\text { How are students encouraged to collaborate with others? }\end{array}$ & $\begin{array}{l}\text { Active and } \\
\text { Collaborative } \\
\text { Learning }\end{array}$ \\
\hline $\begin{array}{c}\text { their practical } \\
\text { potential } \\
\text { interpreted? }\end{array}$ & $\begin{array}{l}\text { In what ways do } \\
\text { practitioners and } \\
\text { students interact? }\end{array}$ & $\begin{array}{l}\text { How often do students discuss grades or assignments with } \\
\text { teachers? } \\
\text { How often are career plans discussed with teachers? } \\
\text { What written or oral feedback do students receive from teachers } \\
\text { regarding their academic performance? } \\
\text { How often do students collaborate with teachers on activities and } \\
\text { projects outside of class? }\end{array}$ & $\begin{array}{l}\text { Student- } \\
\text { Faculty } \\
\text { Interaction }\end{array}$ \\
\hline
\end{tabular}




\begin{tabular}{|c|c|c|c|}
\hline & $\begin{array}{l}\text { To what degree is } \\
\text { the campus } \\
\text { environment } \\
\text { supportive? }\end{array}$ & $\begin{array}{l}\text { How satisfied are students with their time on campus and overall } \\
\text { university experience? } \\
\text { How committed is this program to the success of each student? } \\
\text { What support does the program provide to students? } \\
\text { What support do teachers offer? } \\
\text { What support do students provide to other students? } \\
\text { How does the administration support teachers and students? }\end{array}$ & $\begin{array}{l}\text { Supportive } \\
\text { Campus } \\
\text { Environment }\end{array}$ \\
\hline & $\begin{array}{l}\text { How enriching are } \\
\text { higher education } \\
\text { experiences? }\end{array}$ & $\begin{array}{l}\text { In what ways does learning take place outside of the classroom? } \\
\text { How does the university emphasize diversity and cultural } \\
\text { interactions? } \\
\text { How is technology applied within the classrooms and university? } \\
\text { What opportunities are available in terms of community service, } \\
\text { internships, or study abroad? }\end{array}$ & $\begin{array}{l}\text { Enriching } \\
\text { Educational } \\
\text { Experiences }\end{array}$ \\
\hline $\begin{array}{l}\text { How are the } \\
\text { NSSE } \\
\text { benchmarks and } \\
\text { their practical } \\
\text { potential } \\
\text { interpreted? }\end{array}$ & $\begin{array}{l}\text { How might the } \\
\text { NSSE benchmarks } \\
\text { impact praxis and } \\
\text { quality? }\end{array}$ & $\begin{array}{l}\text { What was your first impression of the NSSE benchmarks? } \\
\text { How would you describe these practices to your students? } \\
\text { Would any practices be inappropriate within this program? } \\
\text { Could any of these practices improve student learning and } \\
\text { program outcomes? } \\
\text { Should the program adopt any of these practices now or in the } \\
\text { future? } \\
\text { What single change to this program would have the greatest } \\
\text { positive impact? }\end{array}$ & $\begin{array}{l}\text { Active and } \\
\text { Collaborative } \\
\text { Learning } \\
\text { Student- } \\
\text { Faculty } \\
\text { Interaction } \\
\text { Supportive } \\
\text { Campus } \\
\text { Environment }\end{array}$ \\
\hline
\end{tabular}




\section{Appendix D: Semi-Structured Interview Protocols}

\section{First Interview Protocol}

1. How would you describe your university experience?

a. What was your goal when you enrolled?

b. Why did you attend the program and university that you did?

c. How challenging or academically rigorous was your program?

d. How would you describe the best and worst teachers you had at university?

e. How did you interact with your teachers?

f. How would you describe the students in your program?

g. How would you describe the best and worst students in your program?

h. How did you interact with other students inside and outside of class?

i. Were there opportunities to apply what you were learning outside of class?

j. What were your assignments, classes, and examinations like?

$\mathrm{k}$. What did you learn over the course of your studies?

1. How did you learn as an undergraduate student?

$\mathrm{m}$. What problems did you encounter as a student?

n. How were those problems resolved?

o. How did the campus environment support you and your learning?

p. What was a critical or defining moment in your undergraduate experience?

2. How would you describe your professional experience as a faculty member and how would you describe your students' experiences?

a. How would you describe yourself as a teacher?

b. What standards exist for teachers and for students?

c. What expectations do you have for your students?

d. How do students interact with one another inside and outside of class?

e. What are your assignments, classes, and examinations like?

f. What teaching methods do you find help students learn best?

g. How is technology incorporated into teaching and learning?

$\mathrm{h}$. What problems do you encounter as a teacher?

i. How do students interact with you as a teacher?

j. How much time do you have to dedicate to research?

k. How would you describe the best and worst students you have had as a teacher?

1. What do students do in between and after classes?

$\mathrm{m}$. How is student feedback on learning and progress measured?

n. What do your students struggle to learn?

o. How challenging would you say this program is today?

p. How would you describe the campus environment today?

q. How does the university encourage and support students and teachers?

r. How would you describe Kyrgyz higher education?

s. What do you think higher education is like in the United States? 
t. How well would you expect US university practices to work here?

\section{Second Interview Protocol}

1. How challenging is this academic program?

a. How much academic effort is required to succeed in the program?

b. What are the expectations for students?

c. What must students do to successfully complete this program?

d. How much time do students spend outside of class preparing, studying, and working on course material?

e. How many papers or reports are required for each class?

f. How does this program emphasize spending time studying and completing academic work?

2. To what extent is learning active and collaborative?

a. How involved are students with their learning?

b. How is problem-solving emphasized?

c. How comfortable are students asking questions during class?

d. How are class discussions conducted?

e. How are students encouraged to collaborate with others?

3. In what ways do practitioners and students interact?

a. How often do students discuss grades or assignments with teachers?

b. How often are career plans discussed with teachers?

c. What written or oral feedback do students receive from teachers regarding their academic performance?

d. How often do students collaborate with teachers on activities and projects outside of class?

4. To what degree is the campus environment supportive?

a. How satisfied are students with their time on campus and overall university experience?

b. How committed is this program to the success of each student?

c. What support does the program provide to students?

d. What support do teachers offer?

e. What support do students provide to other students?

f. How does the administration support teachers and students?

5. How enriching are higher education experiences?

a. In what ways does learning take place outside of the classroom?

b. How does the university emphasize diversity and cultural interactions?

c. How is technology applied within the classrooms and university?

d. What opportunities are available in terms of community service, internships, or study abroad?

6. How might the NSSE benchmarks impact praxis and quality?

a. What was your first impression of the NSSE benchmarks?

b. How would you describe these practices to your students? 
c. Would any practices be inappropriate within this program?

d. Could any of these practices improve student learning and program outcomes?

e. Should the program adopt any of these practices now or in the future?

f. What single change to this program would have the greatest positive impact? 
Appendix E: NSSE Benchmarks Obtained from ("NSSE Benchmarks," 2000)

\section{$\overline{\bar{N}}$ NSSE \\ III \\ Benchmarks of Effective Educational Practice}

The benchmarks are based on 42 key questions from the nsse survey that capture many vital aspects of the

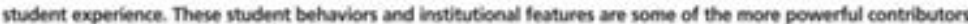
to learning and perronal development.

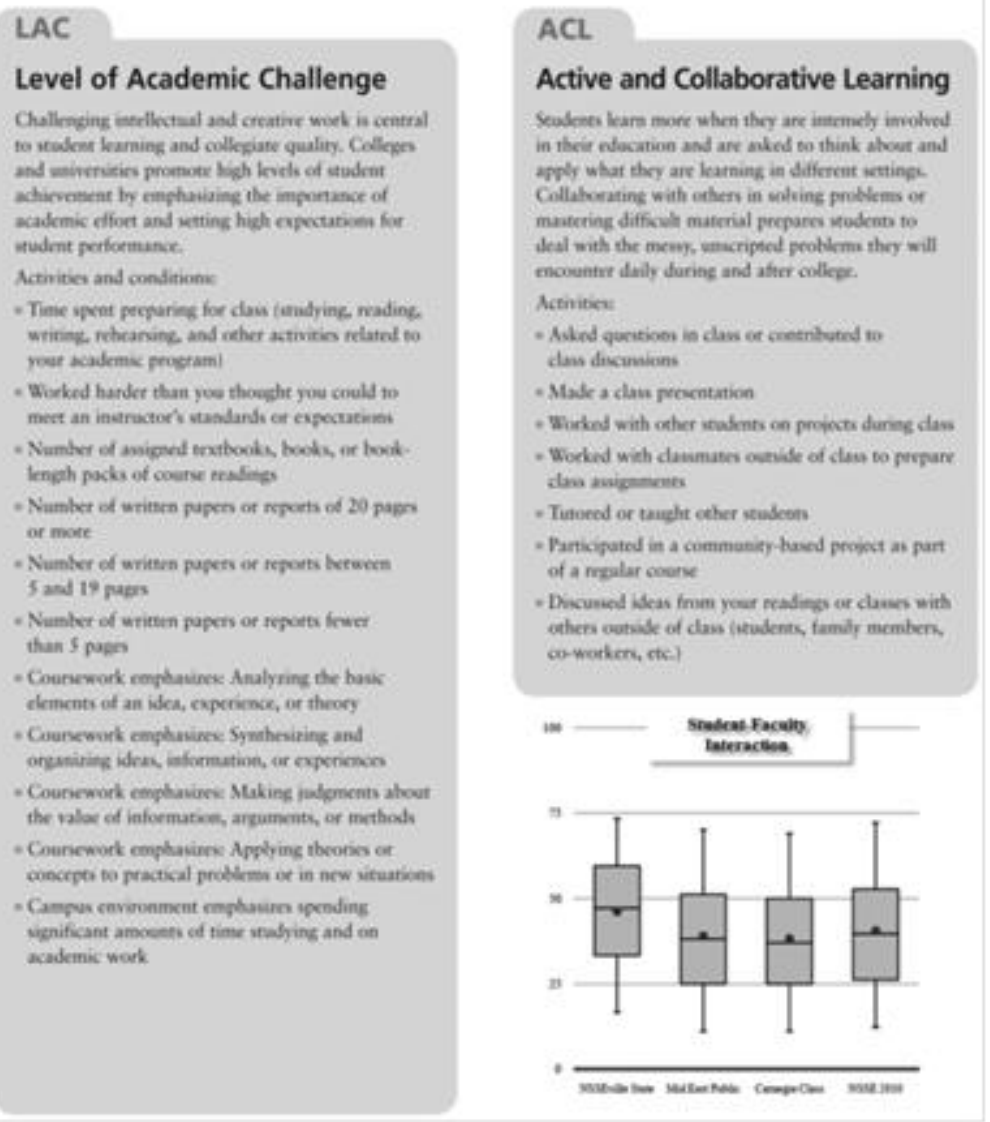

\section{$A C$}

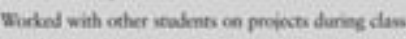

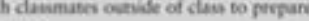

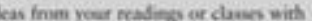

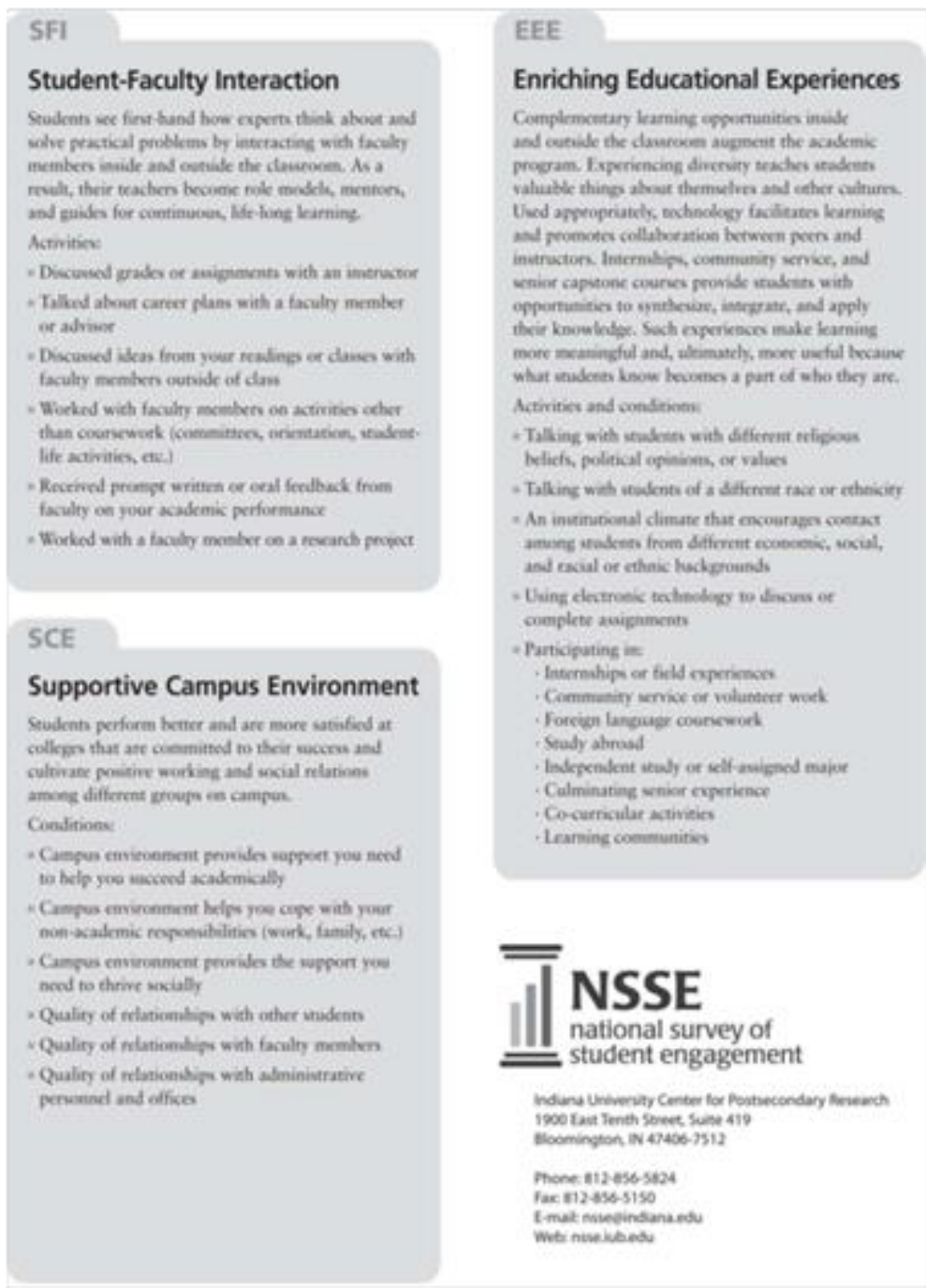




\section{Appendix F: Classroom Observation Instrument}

Adapted from (Harris \& Lane, 2015; NSSE Benchmarks, 2000)

Date of Observation:

Classroom Description:

Course Subject and Attendance:

Instructional Methods:

Notes on Students Being Observed:

Notes on NSSE Supportive Classroom Environment:

\begin{tabular}{|c|c|c|}
\hline \multicolumn{2}{|c|}{ Engaged Student Behaviors } \\
\hline $\begin{array}{c}\text { In-Class } \\
\text { Behaviors }\end{array}$ & Course of Class & NSSE \\
Benchmark
\end{tabular}

\begin{tabular}{|c|c|c|}
\hline \multicolumn{3}{|c|}{ Disengaged Engaged Student Behaviors } \\
\hline $\begin{array}{c}\text { In-Class } \\
\text { Behaviors }\end{array}$ & Course of Class & NSSE \\
Benchmark
\end{tabular}




\begin{tabular}{|c|c|c|}
\hline $\begin{array}{c}\text { Settling in / } \\
\text { packing up }\end{array}$ & $\begin{array}{c}\text { Active and } \\
\text { Collaborative } \\
\text { Learning }\end{array}$ \\
\hline Unresponsive & Active and \\
& $\begin{array}{c}\text { Collaborative } \\
\text { Learning }\end{array}$ \\
\hline Off-task & & Level of \\
& Academic \\
\hline $\begin{array}{c}\text { Disengaged } \\
\text { technology } \\
\text { use }\end{array}$ & Challenge \\
\hline $\begin{array}{c}\text { Disengaged } \\
\text { student } \\
\text { interaction }\end{array}$ & Enriching \\
\hline $\begin{array}{c}\text { Distracted by } \\
\text { another } \\
\text { student }\end{array}$ & Educational \\
& Experiences \\
\hline
\end{tabular}

\begin{tabular}{|c|c|}
\hline $\begin{array}{c}\text { NSSE } \\
\text { Benchmark }\end{array}$ & Notes on Observable Indicators \\
\hline $\begin{array}{l}\text { Level of } \\
\text { Academic } \\
\text { Challenge }\end{array}$ & $\begin{array}{l}\text { Student and Teacher Preparation, Assignment Completion, Brining } \\
\text { Materials, Analyzing, Synthesizing, Judging, Applying Theory to } \\
\text { Practical Problems. Emphasis on Studving and Academic Work }\end{array}$ \\
\hline $\begin{array}{l}\text { Active and } \\
\text { Collaborative } \\
\text { Learning }\end{array}$ & $\begin{array}{l}\text { Asking Questions, Discussing Ideas, Making Class Presentations, } \\
\text { Students Working Together, Instructor Interactions, Assignment } \\
\text { Preparation, Classroom Involvement and Participation }\end{array}$ \\
\hline $\begin{array}{l}\text { Student- } \\
\text { Faculty } \\
\text { Interaction }\end{array}$ & $\begin{array}{l}\text { Teacher Interactions, Asking Students Questions, Discussing Ideas } \\
\text { and Projects, Providing Written or Oral Feedback, Working with } \\
\text { Teachers }\end{array}$ \\
\hline $\begin{array}{l}\text { Supportive } \\
\text { Campus } \\
\text { Environment }\end{array}$ & $\begin{array}{l}\text { Student-Teacher-Administrator Interactions, Sufficient Facilities, } \\
\text { Campus Environment, Learning Support, Non-Academic Support, } \\
\text { Socializing }\end{array}$ \\
\hline $\begin{array}{l}\text { Enriching } \\
\text { Educational } \\
\text { Experiences }\end{array}$ & $\begin{array}{l}\text { Student Diversity, Interactions of Students with Different Genders, } \\
\text { Ethnicities, Races, and Nationalities, Applying Technology, } \\
\text { Participating in Additional Activities }\end{array}$ \\
\hline
\end{tabular}




\section{Appendix G: Preamble}

\section{Exploring Western Evidence-Based Practice in Post-Soviet Higher Education}

2 May 2018

You are being invited to participate in a research study. If you are a faculty member, participation will involve answering questions in both an initial interview and a follow-up interview and being observed during one of your classes after the initial interview. If you are a student, participation will involve answering questions in an interview. Interview questions will address your university experience, and your opinion of higher education practices. This study is conducted by J. L. Mettille of the University of Louisville under dissertation chair Dr. Jacob Gross. There are no known risks for your participation in this research study. The information collected may not benefit you directly but may be helpful to others. The information you provide will be used to explore university experiences in the Kyrgyz Republic relative to educational practices associated with student engagement developed in the United States. Your responses will be recorded and stored on an encrypted laptop and uploaded to an encrypted database. The interviews will take no more than thirty minutes each.

Individuals from the College of Education and Human Development of the University of Louisville and the Department of Educational Leadership, Evaluation, and Organization Development, the Institutional Review Board (IRB), the Human Subjects Protection Program Office (HSPPO), and other regulatory agencies may inspect these records. In all other respects, however, the data will be held in confidence to the extent permitted by law. Should the data be published, the name of this university and your identity will not be recorded or disclosed.

Taking part in this study is voluntary. You may choose not to take part at all. By answering interview questions, you agree to take part in this research study. You do not have to answer any questions that make you uncomfortable. If you decide to be in this study, you may stop taking part at any time. If you decide not to be in this study or if you stop taking part at any time, you will not lose any benefits for which you may qualify. If you have any questions, concerns, or complaints about the research study, please contact: J. L. Mettille at +1 (206) 395-6209.

If you have any questions about your rights as a research participant, you may call the HSPPO at +1 (502) 852-5188. You can discuss any questions about your rights as a research participant privately with a member of the IRB. You may also call this number if you have other questions about the research, and you cannot reach the co-investigator, or you want to talk to someone else. The IRB is an independent committee made up of people from the University community, staff of the institutions, as well as people from the community not connected with these institutions. The IRB has reviewed this research study. 
If you have concerns or complaints about the research or research staff and you do not wish to give your name, you may call 1-877-852-1167. This is a 24-hour hotline answered by people who do not work at the University of Louisville.

Sincerely,
Principal Investigator
Co-Investigator
Dr. Jacob P. Gross
J. L. Mettille

Revised Preamble Version 1.1 May 2, 2018 


\title{
CURRICULUM VITAE
}

\author{
J. L. Mettille \\ College of Education and Human Development \\ Department of Leadership, Foundations, and Human Resource Education \\ University of Louisville \\ JLMettille@gmail.com
}

\section{Education}

\section{University of Louisville}

Ph.D., Higher Education Administration, 2018

Concentration: Educational Leadership, International Education, Student Empowerment Dissertation Title: A Fact to be Coped with over Time: Exploring Western EvidenceBased Practice in Post-Soviet Higher Education.

Chair: Dr. Jacob K. Gross

M.A., Higher Education Administration, 2011

Concentration: Institutional Research, Student Persistence, and Program Evaluation

\section{Saint Louis University}

B.A. Biology \& Psychology, 2007

Honors Thesis Title: Logical Analysis of Proposed Theoretical Evolutionary Species Concepts

\section{Areas of Scholarly Interest}

International systems and practices of higher education. Educational reform and organizational change management. Objectivist philosophy in post-secondary education praxis and research. Student empowerment in developing educational contexts.

\section{Publication}

Kotamraju, P., \& Mettille, J. L. (2012). Using return on investment (ROI) and other related tools: Guidelines for measuring career and technical education (CTE) internal efficiency and external effectiveness. National Research Center for Career and Technical Education, University of Louisville. 